\title{
A characterization of laminar and turbulent boundary layers for use in an investigation of boundary layer state effects on turbine blade film colling schemes
}

Christopher Michael Nestor

Follow this and additional works at: https://researchrepository.wvu.edu/etd

\section{Recommended Citation}

Nestor, Christopher Michael, "A characterization of laminar and turbulent boundary layers for use in an investigation of boundary layer state effects on turbine blade film colling schemes" (2017). Graduate Theses, Dissertations, and Problem Reports. 4013.

https://researchrepository.wvu.edu/etd/4013

This Problem/Project Report is protected by copyright and/or related rights. It has been brought to you by the The Research Repository @WVU with permission from the rights-holder(s). You are free to use this Problem/Project Report in any way that is permitted by the copyright and related rights legislation that applies to your use. For other uses you must obtain permission from the rights-holder(s) directly, unless additional rights are indicated by a Creative Commons license in the record and/ or on the work itself. This Problem/Project Report has been accepted for inclusion in WVU Graduate Theses, Dissertations, and Problem Reports collection by an authorized administrator of The Research Repository @ WVU. For more information, please contact researchrepository@mail.wvu.edu. 


\title{
A Characterization of Laminar and Turbulent Boundary Layers for Use in an Investigation of Boundary Layer State Effects On Turbine Blade Film Cooling Schemes
}

\author{
Christopher Michael Nestor \\ Problem/ Research Report submitted to the \\ Benjamin M Statler College of Engineering \& Mineral Resources \\ at West Virginia University \\ in partial fulfillment of the requirements \\ for the degree of

\section{Master of Science \\ in} \\ Mechanical Engineering
}

\author{
Andrew C. Nix, Ph.D., Committee Chairperson \\ John M. Kuhlman, Ph.D. \\ Cosmin Dumitrescu, Ph.D. \\ Department of Mechanical and Aerospace Engineering \\ Morgantown, West Virginia \\ December 2017
}

Keywords: Gas Turbines, Turbulence, Boundary Layers, Hot Wire Anemometry Copyright (C) 2017, Christopher Nestor 


\section{ABSTRACT \\ A Characterization of Laminar and Turbulent Boundary Layers for Use in an Investigation of Boundary Layer State Effects On Turbine Blade Film Cooling Schemes}

\section{Christopher Michael Nestor}

The boundary layer region of a flow is a primary area of interest when analyzing the effects of various flow phenomena, heat transfer, and skin friction near an immersed object's surface. Characterizing this region yields a proper understanding of mass, momentum, and energy transfer to or from the region near the immersed body surface. Such characterization includes the boundary layer height, displacement, and momentum boundary layer thicknesses among many attributes. These factors offer a description of the flow for a laminar state, but also must include length scales, turbulence intensities, and distribution of turbulent kinetic energy to fully describe a boundary layer in the turbulent regime. This investigation will experimentally find these boundary layer characterization parameters for four scenarios. Two independent flow variations will be introduced and their effects upon the boundary layer examined and compared to previous and theoretical data. The first two scenarios examine the effects of wall suction on and off and the resulting effect on a non-tripped laminar boundary layer. The last two scenarios maintain the cases of suction on and off but introduce a turbulence generator within the boundary layer in order to trip the boundary layer to a turbulent state. Confirmation of turbulence and to what degree the flow is turbulent is found through calculating the turbulence intensity and length scale. This investigation is experimentally conducted in the West Virginia University Turbine Aerodynamic and Advanced Cooling (TAAC) Wind Tunnel facility, which has been recently recommissioned by the author. Pitot-static and hotwire anemometry were used to record data. Freestream turbulence was controlled by a passive turbulence generator and held constant for each scenario. It was found that a non-tripped flow with no suction yielded a boundary layer height of 1.045 inches taken 9.625 inches downstream of the turbulence grid location. Once suction is introduced, this height reduces to 0.795 inches. Similarly, suction decreased the boundary layer height for the tripped boundary flow from 2.25 inches to 1.17 inches. Due to the way the investigation originally defined the boundary layer height at $99 \%$ freestream conditions, conclusions yield that resolutely maintaining this definition slightly deviates this investigation's results from prior benchmarked data prior to facility recommissioning. However, general trends in boundary layer parameters are consistent, and turbulence intensities along with turbulent length scales from the tripped flow scenario validate a turbulent boundary layer. Turbulence intensities and other boundary layer characteristics detail for the non-tripped flow scenarios, which assume a laminar boundary layer, more closely align instead with a transitional flow regime as opposed to a completely laminar one as assumed. Understanding the influence of suction and turbulence within the boundary layer aids this facility's goal of matching flow parameters nondimensionally as experienced on actual turbine blades in the hot section of gas turbine engines. Serving as a new benchmark for this lab, future turbine blade cooling schemes can be tested in nondimensionally matched conditions. While these future experiments will employ advanced nonintrusive measurement techniques, the author utilizes this precursor investigation for conceptual understanding of 
flow field conditions and the influence of parameters such as suction and turbulence on the boundary layer. 


\section{ACKNOWLEDGEMENTS}

I would like to thank my advisor, Dr. Nix for his support and agreement to take me on as a Masters and now a Ph.D. student. Often thrilled to see Dr. Nix listed as an instructor for a course, I always registered for his classes as he frequently made difficult material easier through explanations and content presentation. His passion for the field of gas turbines is rather contagious and ushers a desire for me to make this the field of my profession.

I would also like to thank my committee members Drs. John Kuhlman and Cosmin Dumitrescu. Having both professors for a class in graduate school, I have learned an extraordinarily large amount from both. Able to answer any question I had regarding course content or the discipline of Mechanical Engineering as a whole, they have shown me that there is always more to learn than what appears. I hope to have both of these faculty members on my Ph.D. committee.

Since the start of my academic career at West Virginia University, there has been one person perpetually offering guidance and lending an ear for my triumphs and struggles. This person has become one of my close friends and confidants, as she has now become a mentor in my pursuit of a doctorate. For these reasons, I would like to thank Melissa Morris for all she has done to inspire and guide me.

Starting my graduate academic career, I sought to solicit funding to assist with the financial burden of graduate school. The first opportunity I found was with the Fundamentals of Engineering Program at West Virginia University managed by Michelle Poland. Michelle has been an excellent mentor and source of relentless academic, professional, and personal advice. Always understanding and available, she has worked with me to help balance the time commitment struggle of research, classes, and my funding responsibilities. Her commitment to my ongoing pursuits, and allowing me to be the DogFather's babysitter while traveling commands my sincerest gratitude.

Being able to complete this report to start a doctorate program is in due part to the efforts of undergraduate researchers Richard (Joey) Licata and Charles (Charlie) Hamilton. Without 
their assistance, collecting data would not have been as efficient and swift. As time progresses and we work more together, it is clear that not only their knowledge but character and personalities make even the most monotonous task interesting. I look forward to continuing research with these two gentlemen.

I would finally like to thank my family, Michael, Jayne, and Kathleen Nestor, for always encouraging my ongoing academic pursuits, and always offering assistance; even when not requested. Without them, I would not be able to be where I am today. Additionally, I want to recognize Miranda Masters, who has indelibly strengthened my conviction to continue pursuing a doctorate through her steady support and confidence in me; especially when I myself don't believe. 


\section{Table of Contents}

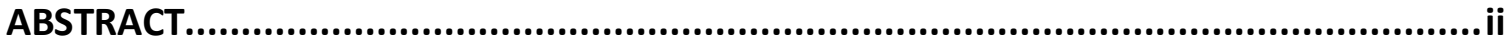

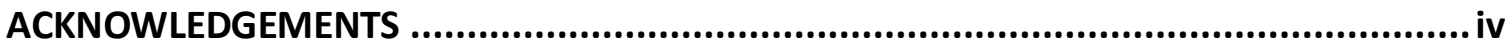

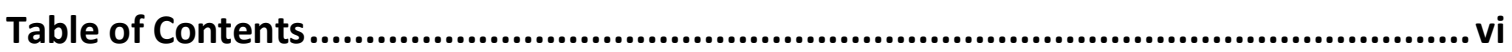

List of Figures...................................................................................................... viii

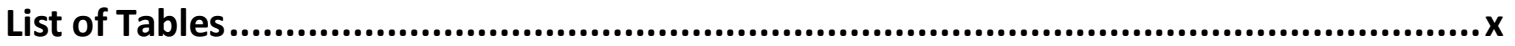

NOMENCLATURE

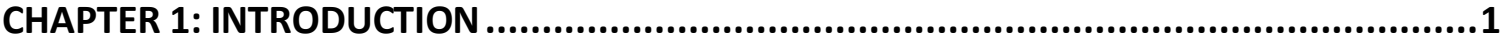

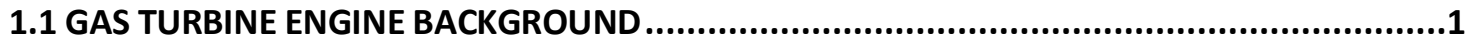

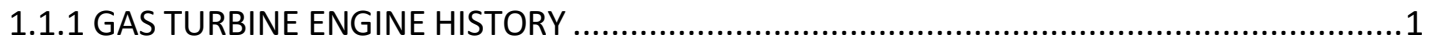

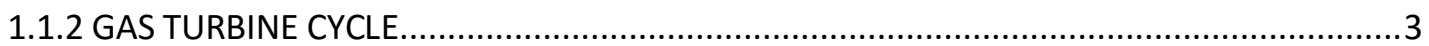

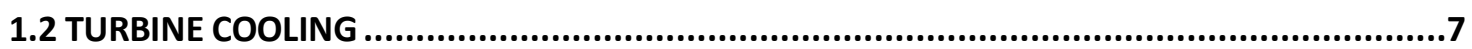

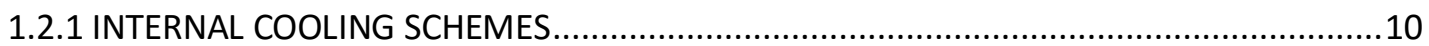

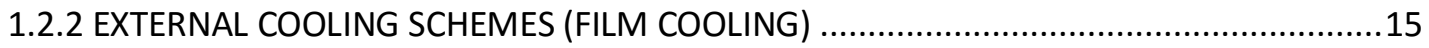

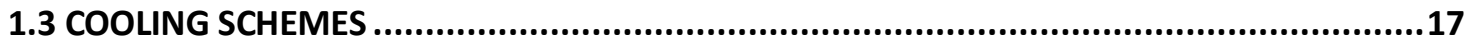

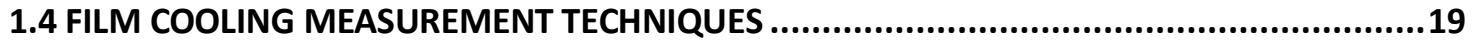

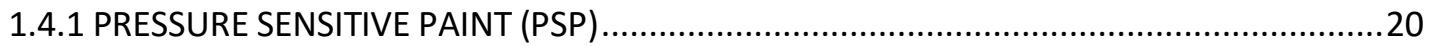

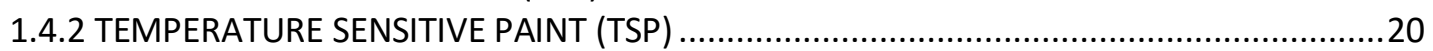

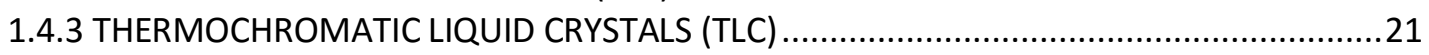

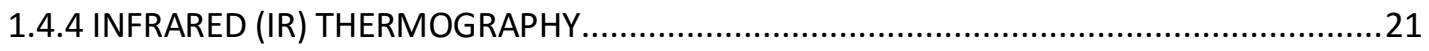

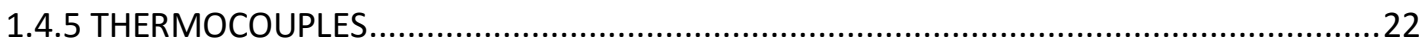

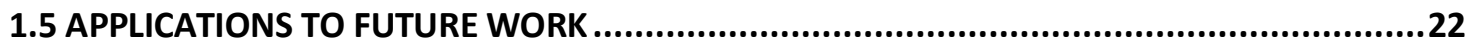

CHAPTER 2: BOUNDARY LAYERS \& FLOW CLASSIFICATION .....................................24

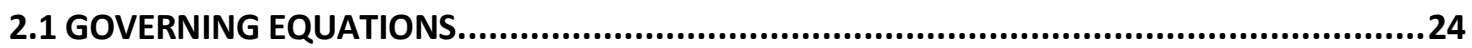

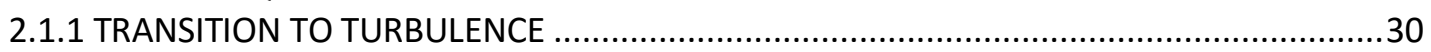

2.2 BOUNDARY LAYER IMPORTANCE

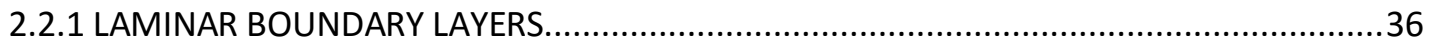

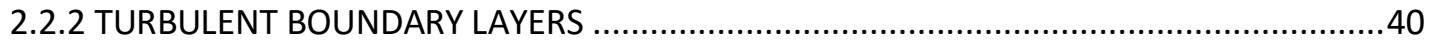

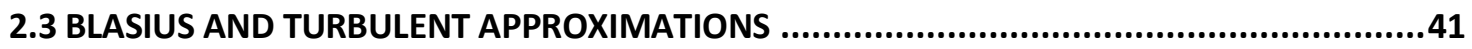

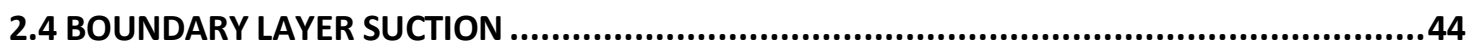

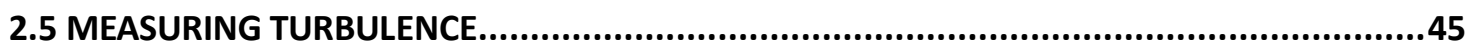

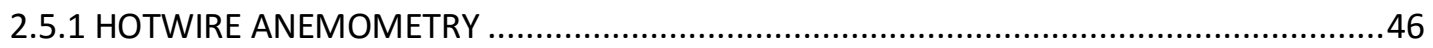

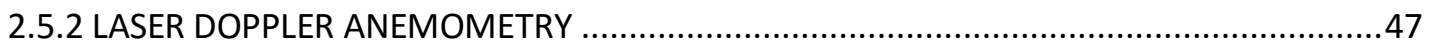

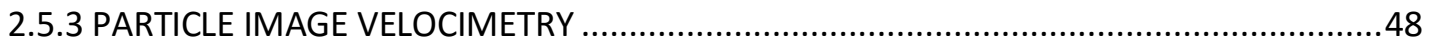

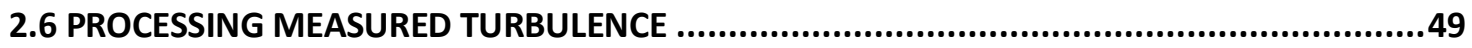

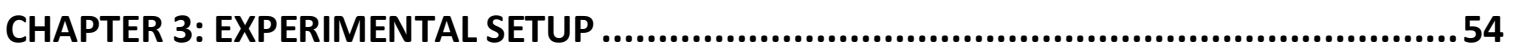

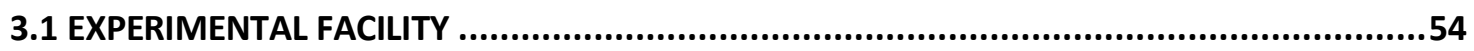

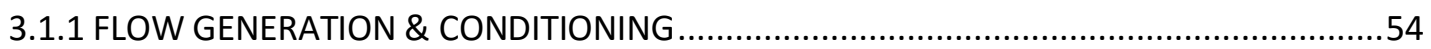

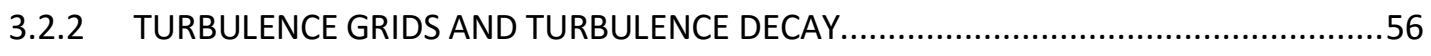

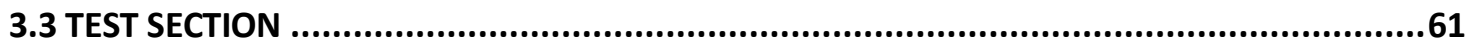


CHAPTER 4: DATA COLLECTION \& CALIBRATION ............................................65

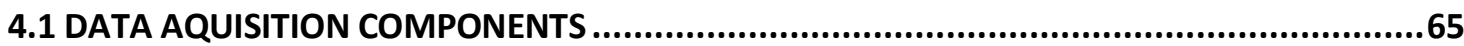

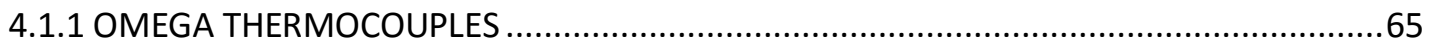

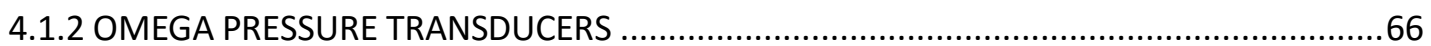

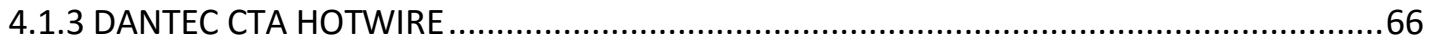

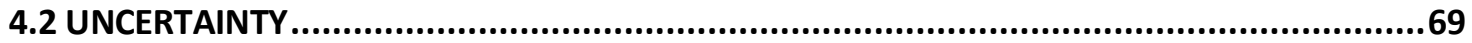

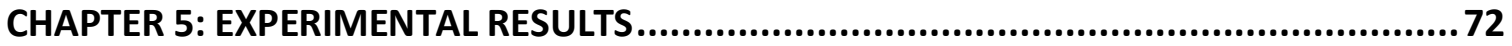

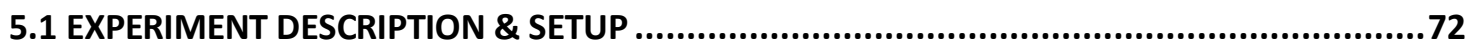

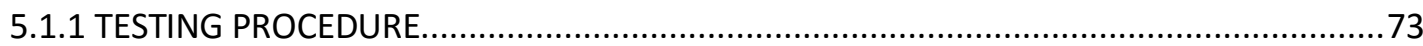

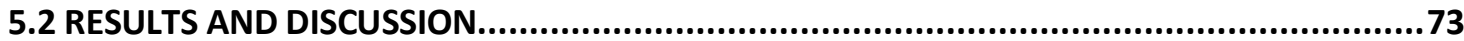

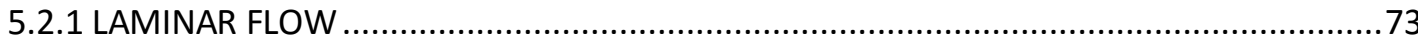

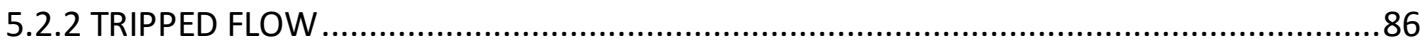

CHATPER 6: CONCLUSIONS AND FUTURE WORK ...............................................97

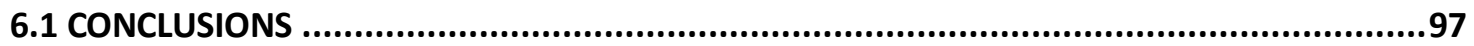

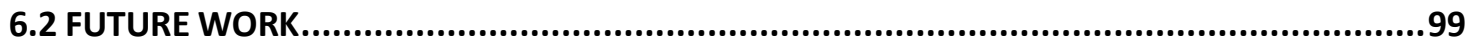

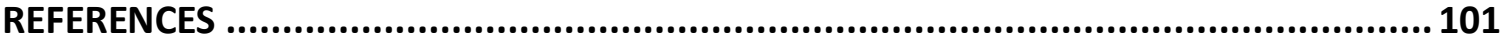




\section{List of Figures}

Figure 1 - Sir Frank Whittle and his Jet Engine [4].........................................................

Figure 2 - P-v Diagram of Ideal Brayton Cycle [6]......................................................... 3

Figure 3 - T-s Diagram of Ideal Brayton Cycle [6] .......................................................

Figure 4 - Combined Brayton \& Rankine Cycles [7] .........................................................

Figure 5 - Brayton Cycle Aircraft Engines [9] .................................................................

Figure 6 - Various Gas Turbine Engine Configurations [10] .............................................

Figure 7 - Turbofan Cutaway with $\mathrm{T}_{4}$ Location and Temperature Gradient [11] ..................8

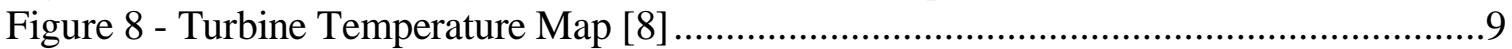

Figure 9 - Turbine Cooling Methods [13] ....................................................................

Figure 10 - Interior Cooling Cut Away [15] .............................................................11

Figure 11 - Flow Regions of Impinging Jet [16] ..........................................................12

Figure 12 - Channel Cooling [8] ...............................................................................13

Figure 13 - Static Turbine Internal Cooling [8]............................................................

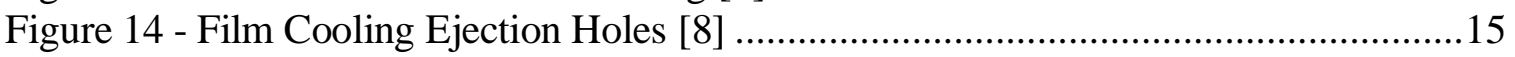

Figure 15 - Film Cooling Temperature Decay of Cooling Jet [19] …….............................16

Figure 16 - Historical Progression of Turbine Inlet Temperatures [22] ..............................18

Figure 17 - Infrared Spectrum [31] .....................................................................22

Figure 18 - Continuity Control Volume [32] …………............................................25

Figure 19: Conservation of Linear Momentum Control Volume [32] ..............................26

Figure 20 - Energy Equation Control Volume [33]........................................................28

Figure 21 - Cylinder Boundary Layer Turbulent Separation [36] .......................................34

Figure 22 - Von Karmen Vortex Shedding [37]..............................................................35

Figure 23 - Distortion of Fluid Particle Entering Boundary Layer [32] ..............................36

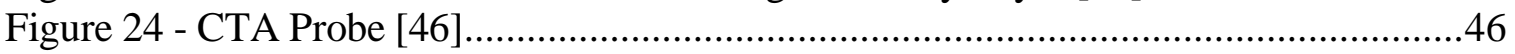

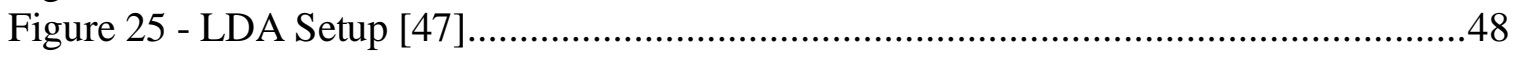

Figure 26 - PIV Setup [48] ……………………………………………………....49

Figure 27 - Time-averaged and fluctuating velocity of turbulent flow [32] .......................50

Figure 28 - Average of Fluctuations and Average Square of Fluctuations Imposed on Signal

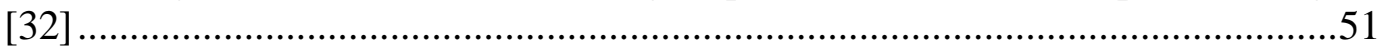

Figure 29 - WVU Wind Tunnel..................................................................................54

Figure 30 - Subsonic Low-Temperature Wind Tunnel ......................................................55

Figure 31 - WVU Subsonic Wind Tunnel at Test Section Exit...........................................56

Figure 32 - Flow through Turbulence Grid [51] ............................................................57

Figure 33 - Non-dimensional Turbulence Intensity and Length Scale for Multiple Bar

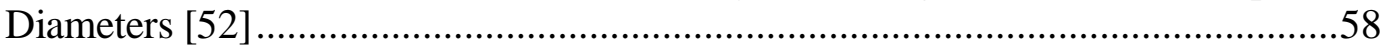

Figure $34-1 / 2$ " and 1/4" Turbulence Grid Geometries ………………….........................59

Figure 35 - 1/4" Grid Nondimensional Turbulence Length Scale [52]................................60

Figure 36 - 1/4" Grid Nondimensional Turbulence Intensity [52] .....................................60

Figure 37 - Test Section Floor ………………………..............................................62

Figure 38 - Suction Slot Setup ...............................................................................63

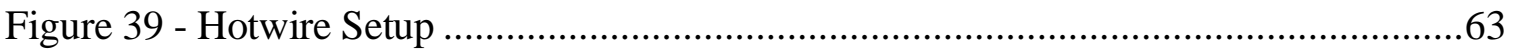

Figure 40 - Data Acquisition System ………………………………………….....65

Figure 41 - Boundary Layer Probe [53] .......................................................................67

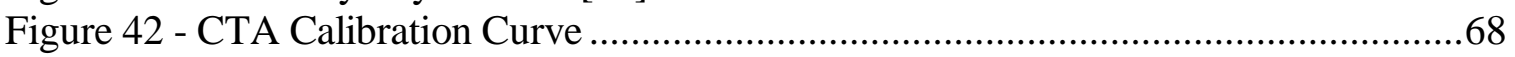


Figure 43 - Suction Slots and Test Region without Probes ............................................73

Figure 44 - Traverse Height Velocity Profile Turbulence OFF and Suction OFF...............75

Figure 45 - Traverse Height Velocity Profile Turbulence OFF and Suction ON................76

Figure 46 - Whitlow WVU Boundary Layer Velocity Profile Comparison [45].................77

Figure 47 - Inside the Boundary Layer Autocorrelation Plot No Turbulence Trip for Suction.

Figure 48 - At the Boundary Layer Autocorrelation Plot No Turbulence Trip...................78

Figure 49 - Outside the Boundary Layer Autocorrelation Plot No Turbulence Trip. .........79

Figure 50 - Power Spectral Density No Turbulence Trip Inside the Boundary Layer.........80

Figure 51 - Power Spectral Density No Turbulence Trip Near the Boundary Layer Suction.

.

Figure 52 - Power Spectral Density No Turbulence Trip Outside the Boundary Layer. .....81

Figure 53 - Averaged PSD for Scenario of No Turbulence Trip Inside the ........................81

Figure 54 - Averaged PSD for Scenario of No Turbulence Trip at the .............................82

Figure 55 - Averaged PSD for Scenario of No Turbulence Trip Outside the ......................82

Figure 56 - Normalized PSD for Scenario of No Turbulence Trip Inside the Boundary Layer

Figure 57 - Normalized PSD for Scenario of No Turbulence Trip at the Boundary Layer .84

Figure 58 - Normalized PSD for Scenario of No Turbulence Trip Outside the Boundary

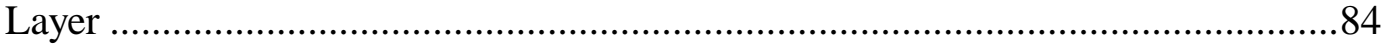

Figure 59 - Theoretical Blasius comparison to experimental scenario of boundary layer non-

tripped flow (a) without suction and (b) with suction ..................................... 85

Figure 60 - Traverse Height Velocity Height Turbulence ON and Suction OFF...............88

Figure 61 - Traverse Height Velocity Turbulence ON and Suction ON ..........................88

Figure 62 - Inside the Boundary Layer Autocorrelation Plot with Turbulence Trip for

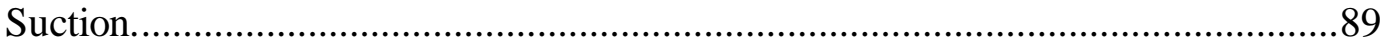

Figure 63 - At the Boundary Layer Autocorrelation Plot with Turbulence Trip for Suction.

.90

Figure 64 - Outside the Boundary Layer Autocorrelation Plot with Turbulence Trip for

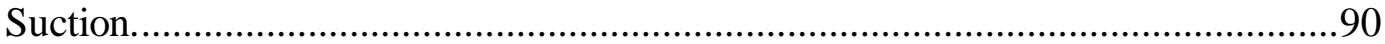

Figure 65 - Power Spectral Density with Turbulence Trip Inside the Boundary Layer......91

Figure 66 - Power Spectral Density with Turbulence Trip at the Boundary Layer.............91

Figure 67 - Power Spectral Density with Turbulence Trip Outside the Boundary Layer....92

Figure 68 - Averaged PSD for Scenario with Turbulence Trip Inside the .........................92

Figure 69 - Averaged PSD for Scenario with Turbulence Trip at the ..............................93

Figure 70 - Averaged PSD for Scenario with Turbulence Trip Outside the ......................93

Figure 71 - Normalized PSD for Scenario with Turbulence Trip Inside the Boundary Layer

Figure 73 - Normalized PSD for Scenario with Turbulence Trip Outside the Boundary Layer

Figure 74 - Theoretical Blasius comparison to experimental scenario of boundary layer tripped flow (a) without suction and (b) with suction ...........................................96 


\section{List of Tables}

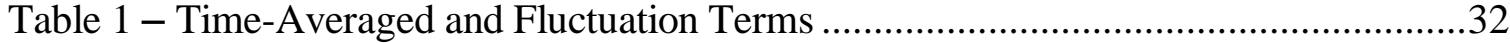

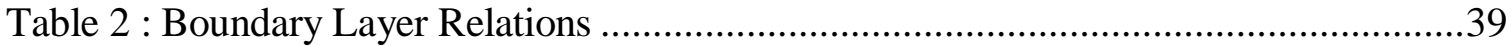

Table 3 - Boundary Layer Approximations ............................................................ 43

Table 4 - Non-dimensionalized Boundary Layer Thicknesses ......................................45

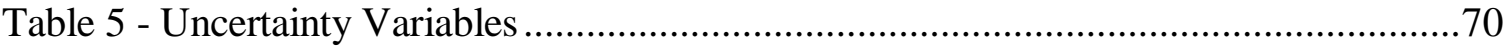

Table 6 - Non-Tripped Laminar Results ................................................................. 74

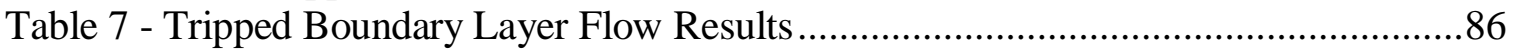


NOMENCLATURE

\begin{tabular}{|c|c|}
\hline $\mathrm{a}$ & conservation law constants \\
\hline A & turbulence length scale constant, amps \\
\hline $\mathrm{b}$ & $\begin{array}{l}\text { Kings Law calibration constant, turbulence } \\
\text { bar width }\end{array}$ \\
\hline $\mathrm{c}$ & Turbulence intensity constant \\
\hline${ }^{\circ} \mathrm{C}$ & Celsius \\
\hline$c_{p}$ & specific heat at constant pressure \\
\hline$C_{f}$ & skin friction coefficient \\
\hline $\mathrm{D}$ & Material Derivative, hole diameter \\
\hline $\mathrm{e}$ & energy \\
\hline$E_{A D}$ & $\mathrm{~A} / \mathrm{D}$ board input range \\
\hline $\mathrm{F}$ & Force \\
\hline g & gravity \\
\hline $\mathrm{h}$ & heat transfer coefficient \\
\hline $\mathrm{H}$ & Shape factor \\
\hline HP & horse power \\
\hline $\mathrm{k}$ & $\begin{array}{l}\text { thermal conductivity, Gaussian coverage } \\
\text { factor }\end{array}$ \\
\hline $\mathrm{kHz}$ & kilohertz \\
\hline $\mathrm{K}$ & kelvin \\
\hline 1 & characteristic length \\
\hline $\mathrm{L}$ & length, hole length \\
\hline $\mathrm{m}$ & meter \\
\hline $\mathrm{mm}$ & millimeter \\
\hline $\mathrm{Nu}$ & Nusselt Number \\
\hline $\mathrm{p}, \mathrm{P}$ & pressure, hole pitch diameter \\
\hline $\operatorname{Pr}$ & Prandtl Number \\
\hline q" & heat flux \\
\hline $\mathrm{Q}$ & generic variable place holder \\
\hline $\mathrm{Re}$ & Reynolds number \\
\hline $\mathrm{R}_{11}$ & autocorrelation value \\
\hline RPM & revolutions per minute \\
\hline $\mathrm{s}$ & entropy, seconds \\
\hline $\mathrm{S}$ & sensitivity factor \\
\hline $\mathrm{t}, \mathrm{T}$ & Time \\
\hline $\mathrm{T}$ & Temperature \\
\hline $\mathrm{Tu}$ & Turbulence intensity \\
\hline $\mathrm{T}_{4}$ & Turbine inlet temperature \\
\hline $\mathrm{U}$ & Velocity \\
\hline
\end{tabular}


GREEK $\mathrm{x}$ component of veolcity

Fluctuating velocity component

y component of velocity

volume, voltage

$\mathrm{z}$ component of veolocity

$\mathrm{x}$ spatial direction

y spatial direction

$\mathrm{z}$ spatial direction

partial derivative

Del Operator

freestream conditions

integral

thermal diffusivity, coolant injection angle coefficient of thermal expansion

boundary layer thickness

displacement thickness

difference of

Streamwise integral length scale

Streamwise integral length scale

Film cooling effectiveness

Adiabatic effectiveness

Kolmogorov length scale

momentum thickness

Wavelength

Dynamic viscosity

kinematic viscosity

density

normal stress, summation

wall shear stress

viscous dissipation

\section{SUBSCRIPTS}

$\mathrm{AD}$

aw

c

calibrator

$\mathrm{f}$

i data acquisition board

Adiabatic condition

Coolant conditions

calibrator value

Film

Coordinate direction parallel with $\mathrm{x}$ 


$\mathrm{j}$
lam
lin
lin
$\mathrm{m}$
$\mathrm{p}$
$\mathrm{RMS}$
static
$\mathrm{std}$
$\mathrm{T}$
total
turb
$\mathrm{w}$
$\mathrm{x}$

\section{SUPERSCRIPTS}

$\mathrm{n}$

,

Coordinate direction parallel with y laminar linearization value linearization from calibration value integral time scale constant pressure property

Root mean square local static condition Standard condition thermal total condition turbulent Wall condition

Streamwise direction

\author{
Turbulence length scale exponent \\ Turbulence intensity decay exponent, \\ Kings Law exponent \\ flux \\ time averaged
}

\title{
ABBREVIATIONS
}

AVH

BR

CAD

CFD

CTA

DAQ

DR

FFT

HPC

HPT

LDA

LED

LPC

LPT

STDV

PIV

PSD

PSP
Anit-vortex hole

Blowing ratio Computer aided design

Computational fluid dynamics constant temperature anemometry Data Acquisition density ratio fast Fourier transform High Pressure Compressor High Pressure Turbine Laser Doppler anemometry Light emitting diode Low Pressure Compressor Low Pressure Turbine standard deviation Particle image velocimetry Power spectral density Pressure Sensitive Paint 


$\begin{array}{cl}\text { RMS } & \text { Root mean square } \\ \text { TAAC } & \text { Turbine Aerodynamic and Advanced } \\ & \text { Cooling } \\ \text { TLC } & \text { Thermochromatic liquid crystals } \\ \text { TR } & \text { temperature ratio } \\ \text { TSP } & \text { Temperature sensitive paint } \\ \text { WVU } & \text { West Virginia University }\end{array}$




\section{CHAPTER 1: INTRODUCTION}

\subsection{GAS TURBINE ENGINE BACKGROUND}

Gas turbine engines have become a dependable energy source for both power generation and aircraft propulsion. As natural gas and other fuel deposits have become abundant and easily extracted, gas turbines have naturally been integrated into the power grid as well as continuing their use for aircraft propulsion. This establishes these engines as an economical standard as society continues to seek for a solution to the ever-increasing demand for energy. Therein, turbine manufacturers continue to strive for higher efficiencies, which will further ensure ongoing socioeconomic reliance on this industry. A historical development and progression of increased turbine efficiency are reviewed in the proceeding section in order to establish validation and necessity for boundary layer characterization pertaining to turbine blade cooling schemes.

\subsubsection{GAS TURBINE ENGINE HISTORY}

The origin of turbine engines can be traced to ancient Egypt where steam was used as a working fluid to rotate a sphere. While many other notable scientists such as Leonardo Da Vinci and Isaac Newton throughout the centuries have contributed toward the development of the modern turbine engine, the true first issued patent for the turbine engine belongs to John Barber in 1791 [1]. His revolutionary design underscored for the first time the fundamental components namely, the compressor, combustor, and turbine sections, and was intended for use with the rapidly advancing technology of the day, "the horseless carriage" [2]. However, due to lack of efficiency in component sections, a full working model was never produced.

Improvements on this patent were not significant until around the turn of the $20^{\text {th }}$ century in Germany when Dr. Franz Stolze mounted both the compressor and turbine on the same shaft. Each component possessed multiple stages, a first of its design [2]. Technological 
inefficiencies plagued this design, however, as the turbine inlet temperature was not able to sustain operation of the thermodynamic cycle for feasible power output.

With the Wright Brothers' development of the first engine powered aircraft and rapidly advancing technology due to military investment from the World Wars of the early to mid $20^{\text {th }}$ century, a new need for aircraft propulsion arose. While almost entirely driven by reciprocating propeller-driven aircraft, flight capabilities of planes had stagnated. During this time Frank Whittle, a British Royal Air Force pilot designed an engine based on the work of his predecessors which featured a multistage compressor and turbine, but which was able to achieve much higher efficiencies. Reassigned to a special duty list while active in the British Air Force, Whittle was able to fabricate and eventually successfully test his engine to be put into production on the Gloster-Whittle E28/39 test plane and later the Gloster-Meteor [3].

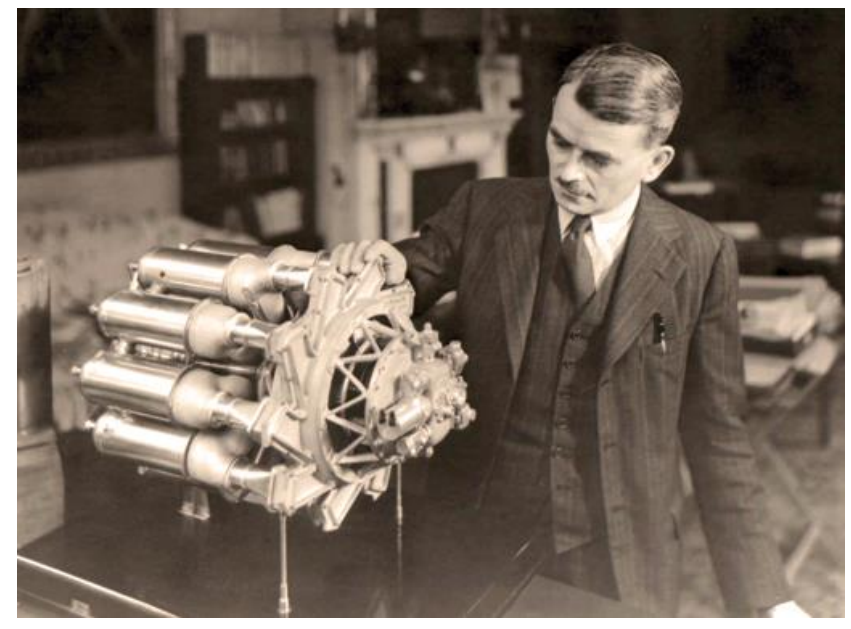

Figure 1 - Sir Frank Whittle and his Jet Engine [4]

Conveniently, and unknowingly occurring in Nazi Germany at the same time as Sir Frank Whittles work, Hans von Ohain was also in the process of developing the jet engine. He also saw the need to increase the ability of these engines by increasing the thrust to weight ratio. His jet design was tested in 1939, three years before Whittle, and was flown on the Heinkel HE 178 aircraft [3]. While von Ohain's work preceded Whittles, which is significant that since air superiority during World War II was critical, Whittle openly shared his research 
with the British Royal Air Force and the United States based company General Electric. Shown in Figure 1 is Whittle's engine prototype as looked upon by Sir Whittle himself.

Further development in turbine engines then shifted to development in the United States when Pratt and Whitney were able to solve the high fuel consumption problem by incorporating two independently rotating compressors and two turbines [5]. This idea paved the way for what is now standard on high-efficiency turbine engines where the inner compressor is powered by the inner turbine, and likewise for the outer compressor and turbines yielding required pressure ratios without sacrificing fuel and power losses.

\subsubsection{GAS TURBINE CYCLE}

Ranging in applications from aforementioned aircraft use to electric power generation, marine and locomotive propulsion, gas turbine engines have a high power to weight ratios which make them ideal for these purposes. This coupled with their ability for a variety of mounting situations and fuels allow them to operate effectively and reliably where reciprocating diesel or gasoline engines cannot.

Gas turbine engines differ fundamentally from reciprocating engines on both physical components, but chiefly on the thermodynamic cycle to which they operate. Known as the Brayton cycle, as shown in Figures $2 \mathrm{a}$ and $2 \mathrm{~b}$, this thermodynamic process describes the conditions of an ideal gas turbine engine cycle.

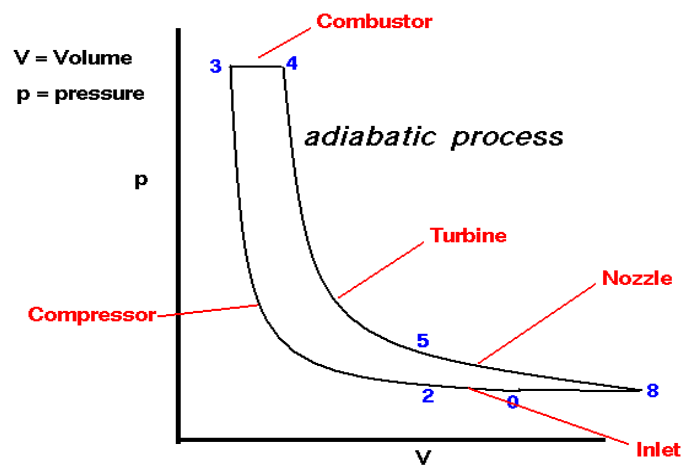

Figure 2 - P-v Diagram of Ideal Brayton Cycle [6]

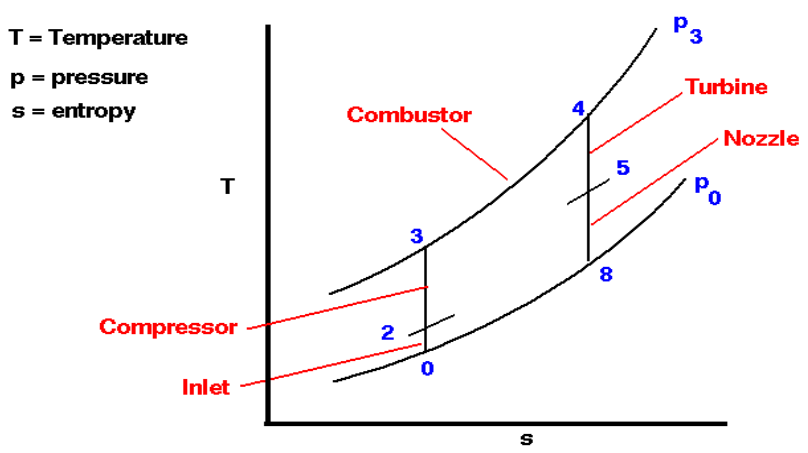

Figure 3 - T-s Diagram of Ideal Brayton Cycle [6] 
The Ideal Brayton Cycle is comprised of four internally reversible processes as follows:

1) Isotropic compression completed via the compressor

2) Constant pressure heat addition completed via the combustion chamber

3) Isotropic expansion completed via the turbine

4) Constant Pressure heat reject completed at the exit of the engine

The working fluid in this open cycle is first drawn into the compressor. After several stages of compression, energy is added to the system by adding fuel then igniting it within the combustor. Without this step, the expansion that takes place in the turbine would equal that absorbed by the compressor assuming no losses. It is due to these losses associated with friction, shaft rotation, heat and pressure drops, that the Brayton cycle in actual applications requires more work done by the compressor and less work produced by the turbine. Therefore, the goal of the most current ongoing research is to yield higher pressure ratios from the compressor reducing fuel flow required and thereby overcoming shortfalls of irreversibilities associated with gas turbine engine operation.

A similar cycle commonly used in power plant applications is the slight modification of the Brayton cycle where the addition of the Rankine Cycle is added to the exhaust. The Rankine cycle, while not important to the Brayton cycle of the gas turbine engine, it is important to note that it operates by heating a fluid, often water in power generation applications, in a boiler from the Brayton cycle exhaust. Then isotropically expanding the working fluid in an independent turbine, condensing the fluid to a compressed liquid, compressing the fluid, and then returning to the boiler where the gas turbine engine exhaust begins the cycle again. This operation is depicted in the schematic of Figure 4. 


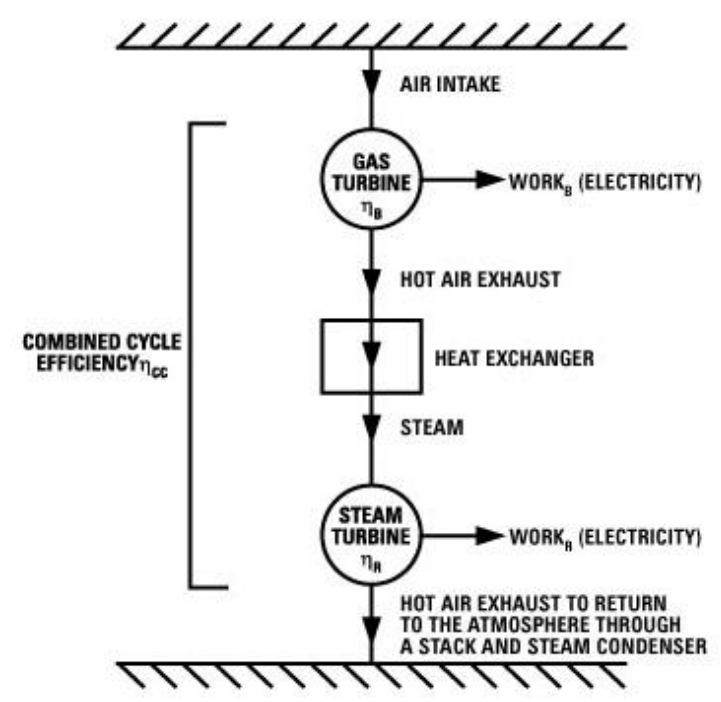

Figure 4 - Combined Brayton \& Rankine Cycles [7]

In regards to aircraft propulsion, there are three main types of engines that operate on the Brayton cycle. Illustrated are the three engines in comparison to the standard open Brayton Cycle: turbojet, turbofan, and turboprop following Figure 5b, 5c, and 5d respectively. While typically arranged in the general order as depicted in Figure 5a, there are modifications such as additional compressors and turbines that these configurations utilize. With the need for additional shaft work by the engine to drive propeller or fan, the compressor and turbine are divided into sections, i.e. a low-pressure turbine (LPT) powering a low-pressure compressor (LPC) and a high-pressure turbine (HPT) powering a high-pressure compressor (HPC). This allows the HPC to operate at a higher shaft rotational speed than the LPC spool independently [8].

The turbojet was the first to be developed and most closely models that of its predecessors, such as the early one developed by Frank Whittle. Thrust in this type of engine is exclusively through expanded gases after the turbine section and through a nozzle. Capable of producing enough thrust alone, application depending, an afterburner will be added to achieve supersonic flight. An afterburner simply adds more fuel to the exhaust nozzle stream 
and ignites increasing the energy output. In modern aviation though, this engine has become eclipsed in implementation for more favorable turbofan engines.
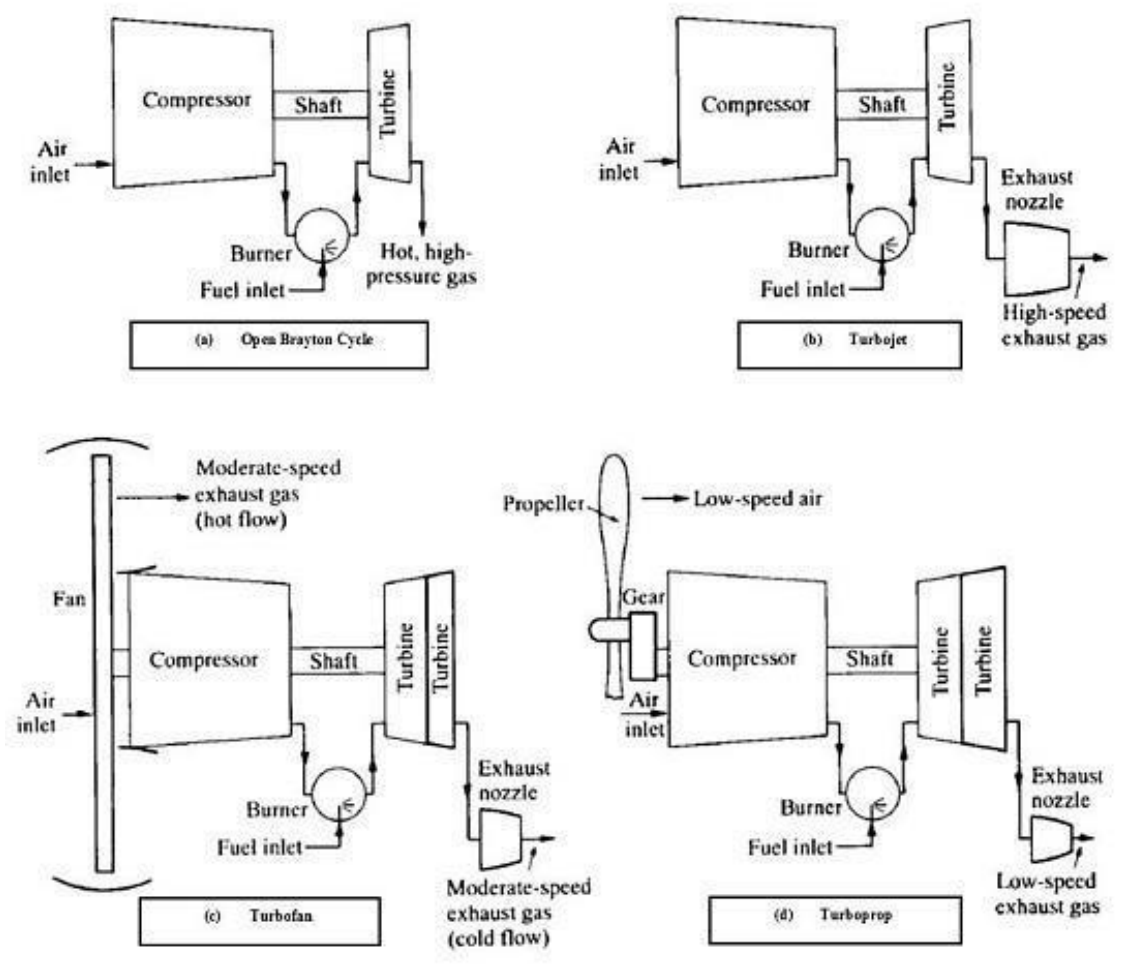

Figure 5 - Brayton Cycle Aircraft Engines [9]

The turboprop engine adds an additional turbine to the system which powers a propeller mounted on a coaxial shaft. The advantages of this engine are high propulsive efficiency, coming however at a sacrifice to limited speed applications. This limitation is due to the propeller exposure, potential for fluttering, and decreased stability occurring when the tips of the blades reach supersonic speed while the base of the blades does not. Conversely, the turboprop configuration is more fuel efficient since the propeller doesn't require a large amount of power from the turbine, and can move slower to maintain thrust due to the blade rotational surface area. 


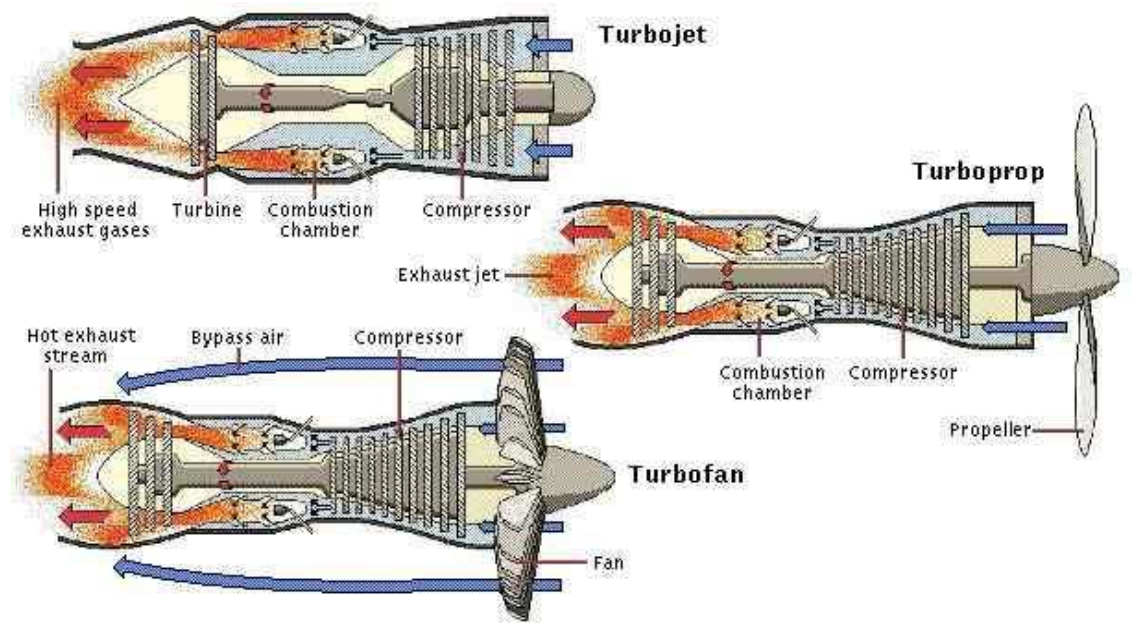

Figure 6 - Various Gas Turbine Engine Configurations [10]

As quickly alluded to, most modern aviation engines are turbofans. In this configuration, a low-pressure fan supplies air to the core but also around the core through a bypass duct. This fan is enclosed in the nacelle of the engine, with narrow tip clearance in order to maximize thrust output. The majority of the thrust of this engine is provided by the fan. The core is the same configuration as that of a turbojet, providing thrust while also powering other system components. The design reduces noise while also increasing the propulsive efficiency of the engine. This engine is more commonly used in commercial aviation with a high bypass duct and military implementation utilizing a low bypass configuration. Figure 6 depicts the differences between all three engine configurations visually.

\subsection{TURBINE COOLING}

Current research in turbine development focuses on increasing the efficiency of the turbine itself. In order to accomplish this, two primary methods are used: increase the compressor pressure ratio and increasing the exit temperature from the combustor into the turbine section. These contribute to higher efficiencies, thus promoting the ability to achieve higher operable turbine inlet temperatures. This temperature location is labeled as "T4" as demonstrated in Figure 7. Represented here is a turbofan engine detailing the temperature 
gradient through the engine until reaching the turbine inlet where expansion begins to cool the fluid.

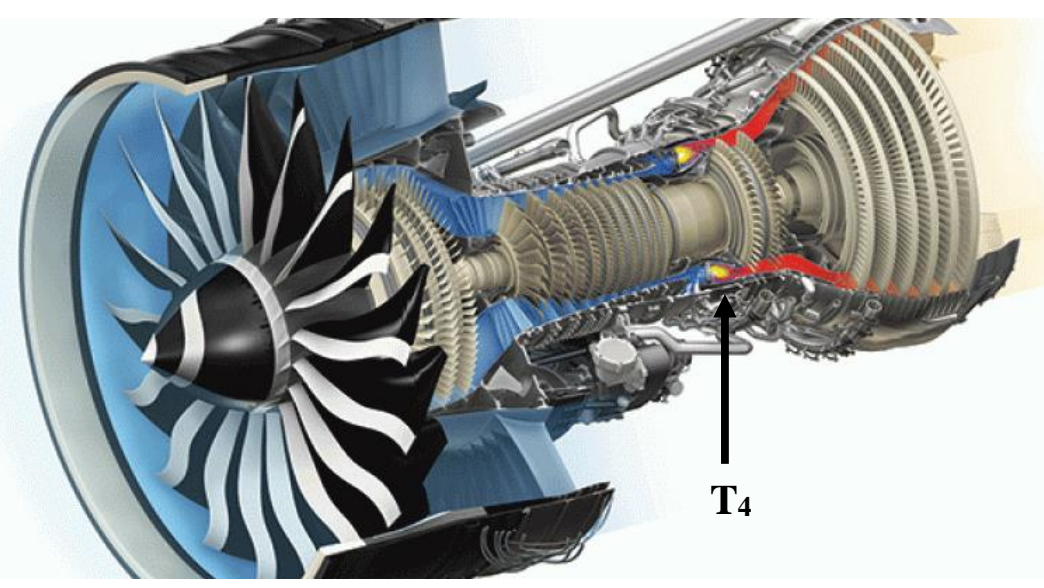

Figure 7 - Turbofan Cutaway with $\mathrm{T}_{4}$ Location and Temperature

Gradient [11]

The bluer region from left to right represents the cooler air as it enters the engine. The hotter air is depicted as the red region starting at the location of $\mathrm{T}_{4}$. It is in this region that the melting point of many component materials is exceeded in the attempt to achieve these higher efficiencies. To alleviate this issue cooling schemes must be employed to attempt to reduce the component surface temperatures. Methods such as advanced blade manufacturing with metal matrix composites also allow the ability to operate at higher temperatures, but these materials as opposed to traditional materials with thermal barrier coatings can be costly.With freestream temperatures at the turbine inlet reaching approximately $2000 \mathrm{~K}$ in advanced military engines and $1600 \mathrm{~K}$ in land-based applications, this is far above the melting temperature of any current ceramic coated super alloy [12]. Exceeding this temperature, the components can and typically do fail due to thermal and mechanical fatigue, creep, oxidation, and corrosion if not cooled. In order to ensure lasting durability in these components, determining temperature distributions to know temperature 
loading and cyclical fatigue can allow blade design to address and mitigate component degradation due to operating conditions.

While seeming trivial to cool the components, namely the turbine blades, should the cooling be improperly employed, the higher efficiency desired will not be obtained and additional losses will be experienced. It has been proven that liquid cooling systems are not practical due to impracticalities associated with corrosion and providing adequate surface cooling area exposure [13]. The only method successfully employed in production engines has been internal, forced convection, air cooling or external film cooling when used in conjunction with thermal barrier coatings.

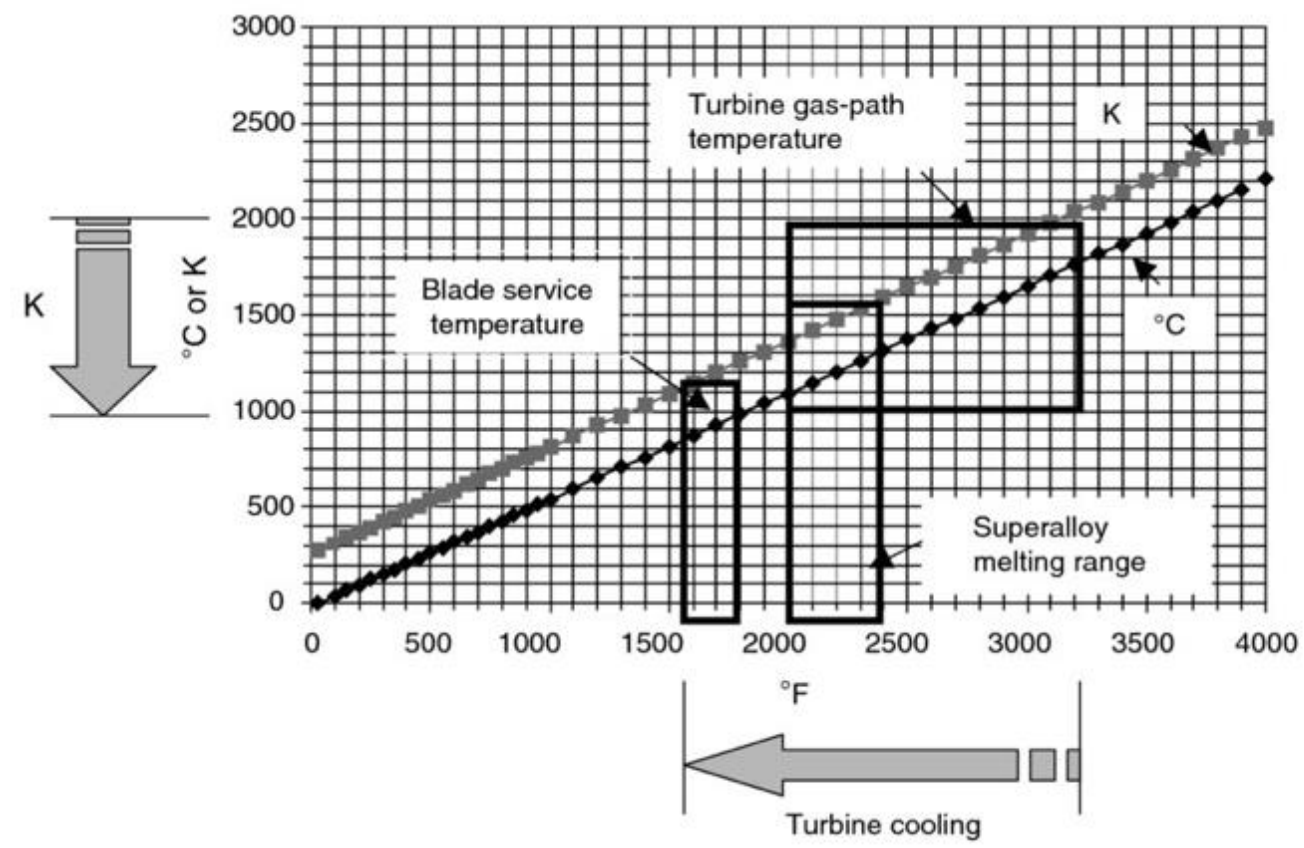

Figure 8 - Turbine Temperature Map [8]

Figure 8 details three distinct temperature ranges: the turbine gas-path temperature which is the hot gas temperature, the super alloy melting range which illustrates the melting point temperatures for modern nickel-based super alloys, and the blade service temperature representing the blade surface operating temperature. Therefore, the bleed air off the compressor is used in the first few stages of the HPT and is relatively cooler roughly 
averaging around $800 \mathrm{~K}$ upon introduction to the hot surfaces [8]. Summarized in Figure 9 below are both internal and external cooling techniques utilized and discussed in this section. More detail regarding why compressor bleed air is used for cooling is included in the proceeding section.

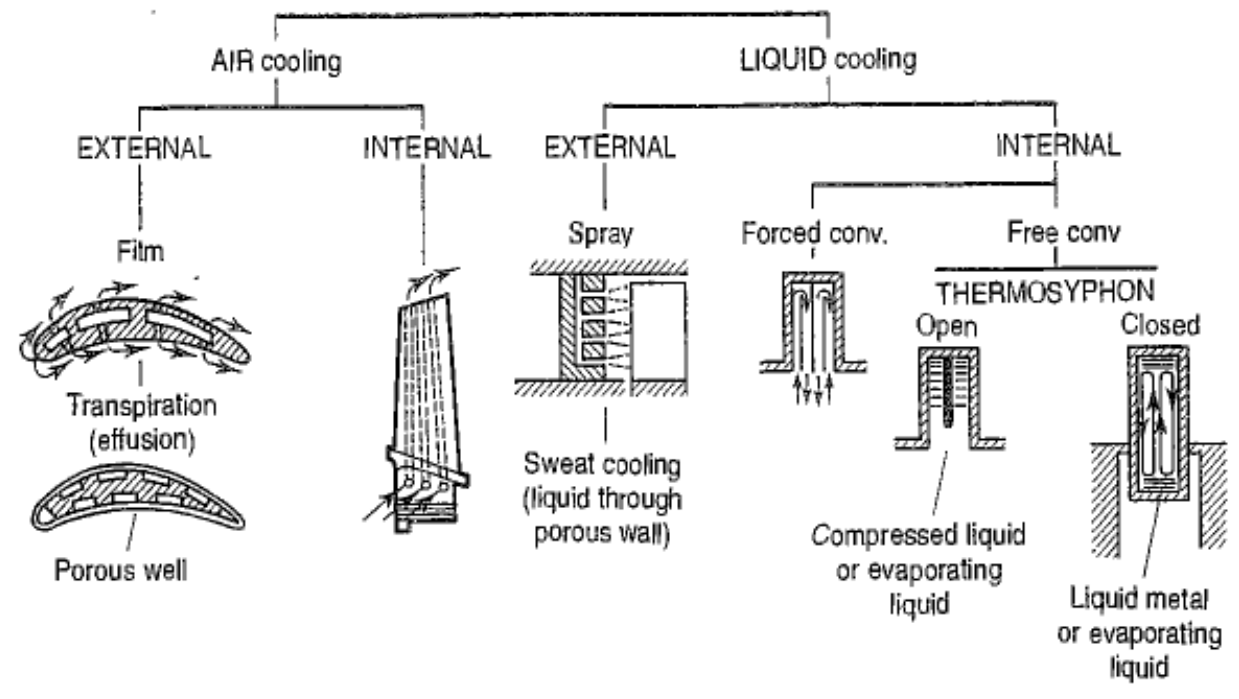

Figure 9 - Turbine Cooling Methods [13]

\subsubsection{INTERNAL COOLING SCHEMES}

Internal cooling techniques route the relatively cooler air from the compressor into the base of the turbine blades through passages in order to cool the turbine blade internally. The primary goal of this setup is to increase the convective heat transfer coefficient by introducing turbulence via turbulators, pin fins, and other structures. Turbulence has been shown to increase the heat transfer, thus is a preferred flow condition for internal cooling [14]. The techniques which incorporate this air cooling include manufacturing various channels throughout the blade commonly with dimples or interior fins, and aiming jets of coolant flow at the blade's front, inside surface. These techniques, known formally as channel and impingement cooling respectively, encompass a great amount of current turbine cooling research. An example of each cooling technique can be found in Figure 10 below with specifics of detailed in Sections 1.2.1.1-1.2.1.2. 


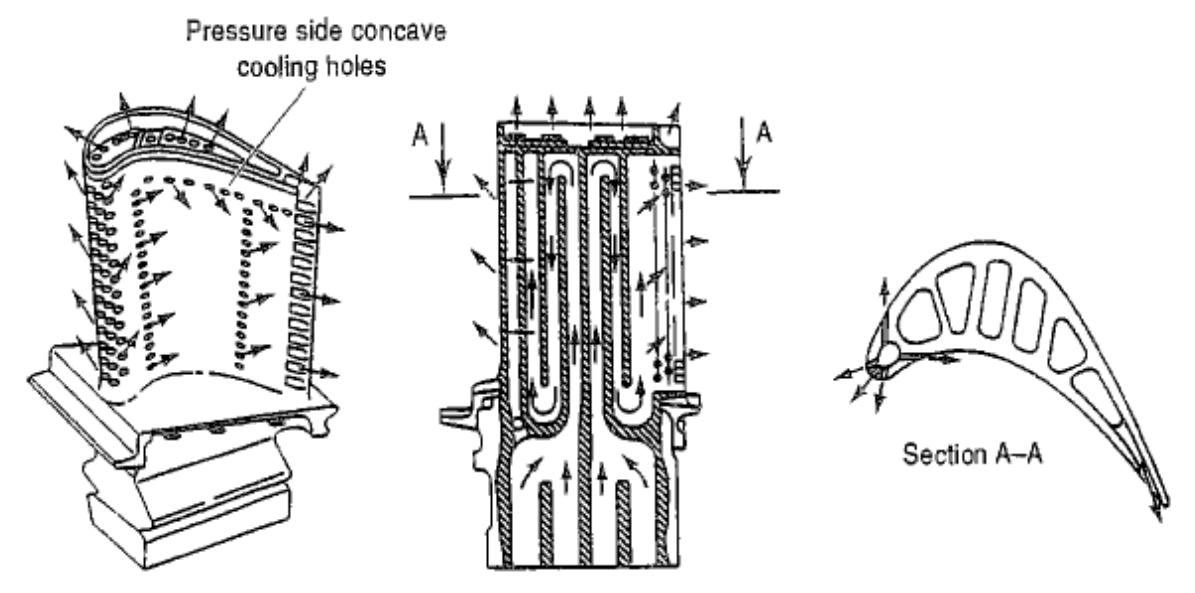

Figure 10 - Interior Cooling Cut Away [15]

\subsubsection{IMPINGEMENT COOLING}

Impingement cooling is an internal cooling technique used in modern turbines to cool the inside surface of turbine airfoils. In this method, the internal cooling air flows perpendicularly to the internal passage that it is cooling, differentiating it from the channel cooling methods that use parallel flow to the surfaces being cooled. Zuckerman, et al. claim that impingement has been shown to produce heat transfer coefficients up to three times those of parallel cooling flow schemes [16]. This is largely due to the much thinner boundary layers created during impingement, and the encouragement of turbulence in the surrounding fluid by the impinged flow [16].

Bleed flow from the high-pressure compressor (HPC) stage is routed through the turbine and into the blade. Jets are typically configured in an array using either cylindrical or slotted holes. The coolant flow is pushed through these constrictions by the higher pressure air at the HPC. The flow leaves the constriction as a free jet. The velocity profile continues to expand further from the jet as the shear layer grows and the free jet decays. Directly in-line with the jet hole, a stagnation region will form on the target surface and a wall jet will disperse radially. As the wall jet from adjacent impingement jets interacts, a fountain region forms, 
pulling the now hotter fluid away from the target. A schematic of the jet flow structure is given in Figure 11.

Jet impingement cooling is currently the most effective technique for increasing the local heat transfer at a surface [15]. Challenges to impingement cooling in turbine blades include potential degradation of structural strength due to hardware changes necessary for implementing jet impingement devices, jet-to-jet interaction based on array spacing, and loss in effectiveness due to the distance from target [15]. For these reasons, it is typically only used toward the front of the blade where the thermal loading is greatest.

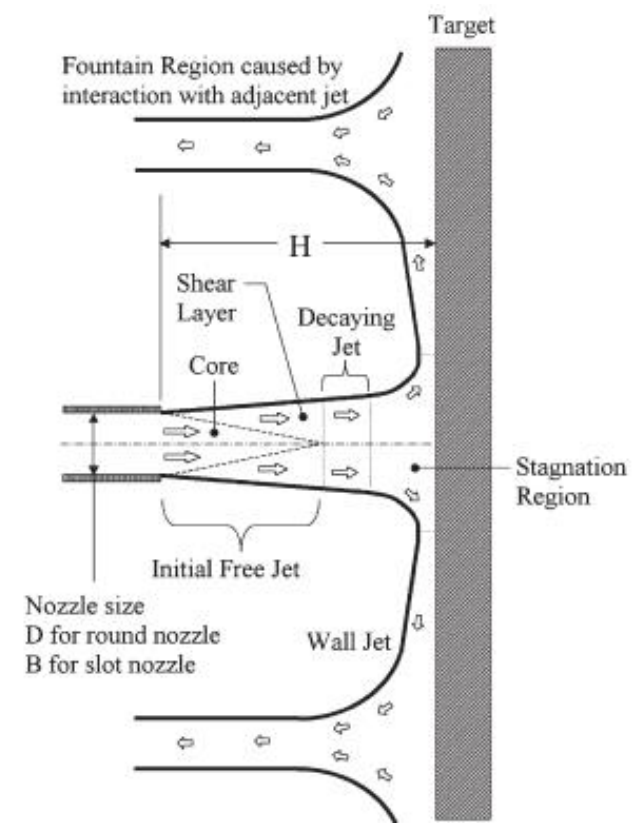

Figure 11 - Flow Regions of Impinging Jet [16]

Current research efforts in jet impingement cooling include hole shape and orientation, target shape, and jet array configuration. Brakmann, et al. found that adding cubic pin fins to the target surface increased the heat transfer rate while minimally impacting the pressure loss [17]. Many other similar experiments have been done to determine the most effective target surface. Most of these studies also incorporate variability in the jet-to-target distance, non- 
dimensionalized by jet diameter, because this detail greatly impacts the flow structure of the jet.

The largest obstacles to jet impingement research are the complexity of turbulence models, meshing difficulties on complex target surfaces, and experimental limitations. Computational studies are additionally limited in complexity due to difficulty modeling flow entrainment interaction [18].

\subsubsection{CHANNEL COOLING}

Channel cooling is among the innovative approaches currently employed for internal cooling of operational turbine airfoils [19]. Starting in 1961, the first cooled turbine blade was rather modest when compared to today's cooling channels which require advanced manufacturing techniques [13]. Channel cooling involves creating a channel within the turbine blade, hence creating an increased internal surface area of contact, for a cooling fluid to flow. In most cases, the flow will be tripped into a turbulent state to improve the heat transfer since a turbulence flow results in higher convective heat transfer coefficient values. Such an example of cooling channels is visually depicted in Figure 12 below designated with arrows. The arrangement of the passages shown in the figure dictates the amount of coolant to the desired section of the blade (i.e. front, back or trailing edge sections).

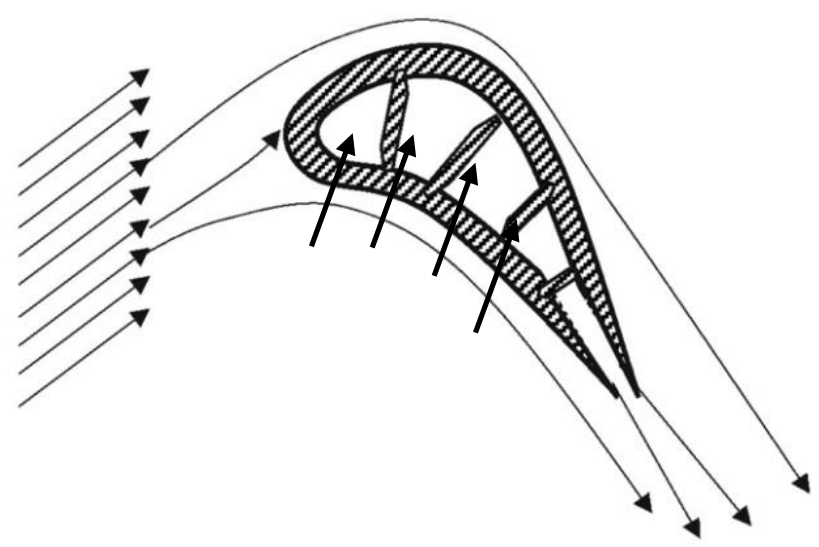

Figure 12 - Channel Cooling [8] 
Among the variations to this method of cooling involves the inclusion of specific vortex creating devices within a channel to help induce more turbulence. Turbulators placed transversely to the flow direction within a channel, are one of such ways this increased heat transfer is accomplished. Existing research on various shapes, orientation, segmentations, and sizes of these turbulators have solidified the positive impact of this device on internal convection cooling. Additionally, adding pin fins at the trailing edge of the turbine airfoil acts in a similar way as turbulators, designed to increase heat transfer by inducing turbulence and increasing conductive surface area. Reversely, it has been found that placing small dimples synonymous to the shape of a golf ball allows flow to detach from the surface and reattach with vortices to enhance the mixing and therefore the heat transfer coefficient. Shown in Figure 13 below is the temperature distribution for a cooled and uncooled turbine blade. The dashed line in the figure shows the temperature profile for the uncooled blade.

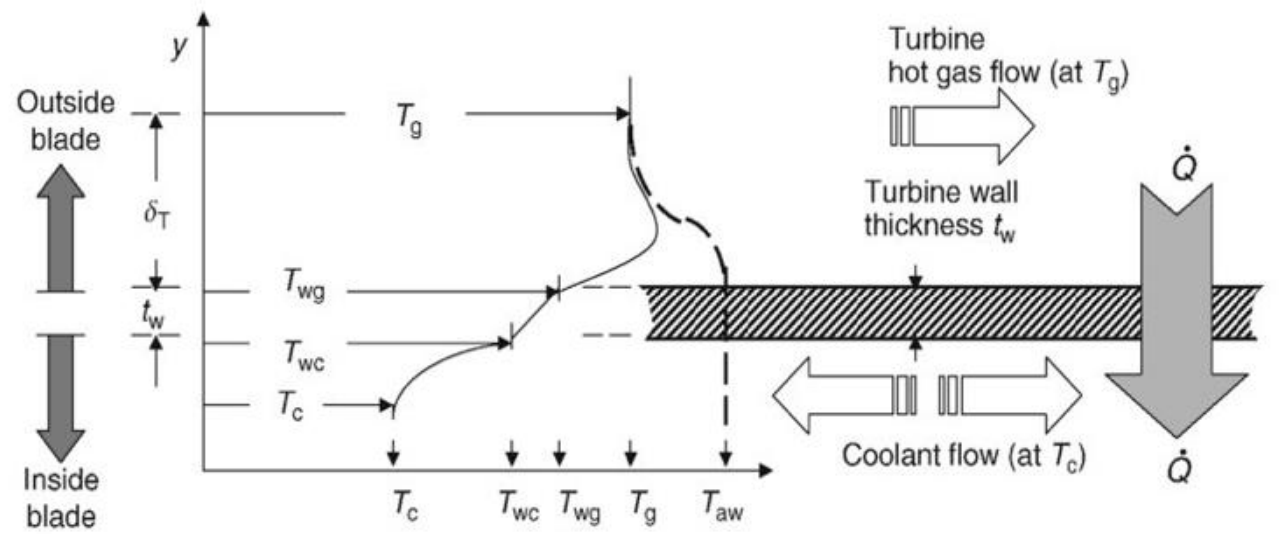

Figure 13 - Static Turbine Internal Cooling [8]

One of the main issues found with channel cooling is that of achieving adequate cooling at the trailing edge of the blade [19]. By increasing the pressure within the blade, designers can increase the flow rate of the coolant, however, this comes from detrimental effects to the performance and efficiency on the compressor. There have been many experiments conducted on how to alleviate this while still maintaining the cooling effectiveness the channels provide. Such studies include varying the flow convergence and turning in the channels, adding dimples to further trip the flow to turbulence, and enacting swirl to further 
promote propagation of cooling down the passages as previously mentioned [20]. The concept of channel cooling dovetails quite closely with that of film cooling as many of the film cooling holes bleed out of these channels or side channels.

\subsubsection{EXTERNAL COOLING SCHEMES (FILM COOLING)}

In contrast to internal cooling, the turbulence which was discussed as pivotal for increased heat transfer can become is detrimental to the component cooling by inducing a surface heat load [9]. The primary method involved with external cooling is film cooling where a stream of coolant air is ejected at specific locations through discrete holes in the surface along the turbine blade thus developing a thin layer of air along the surface. This layer acts as an insulator between the blade surface and the hotter freestream gases. Film cooling is utilized in practically every blade in the turbine section of the gas turbine engine. Such surfaces of the airfoil blades are the leading edges, blade tips, root bases, and main bodies. As found the operating temperature of the gas turbine engine often exceeds the melting temperatures of the blade metals. The goal of film cooling is to reduce the fluid temperature near the airfoil surface. Figure 14 below shows this cooler air is injected into the freestream and how the boundary layer is affected.

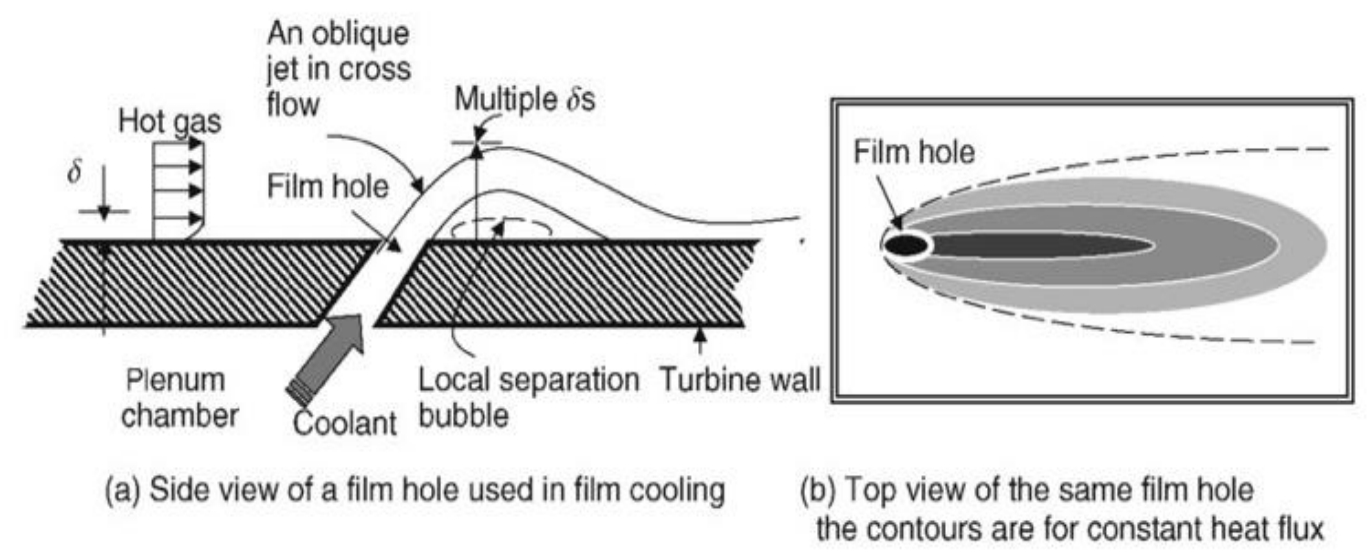

Figure 14 - Film Cooling Ejection Holes [8] 
The cooler air bled off the compressor greatly alters heat transport and momentum inside the airfoil boundary layer. Additionally, other important affecting variables include the shape of the cooling holes, freestream turbulence, and cooling jet-to-mainstream ratios of density, velocity, and mass flux [13]. For turbine blades, it is found that the surface curvature and hole shape play a key role in influencing the heat transfer effectiveness of the film cooling scheme [12]. Adiabatic film cooling effectiveness, a key measurement for low-temperature testing (in relation to actual engine conditions), is defined as the following:

$$
\eta_{a w}=\frac{T_{\infty}-T_{a w}}{T_{\infty}-T_{c}}
$$

This equation details, $T_{\infty}$, as the freestream flow temperature, $T_{a w}$, as the adiabatic wall temperature, $T_{c}$, the coolant flow temperature, and $\eta_{a w}$, the film cooling effectiveness for an adiabatic wall. This equation does not account for any radiation heat loads, and where the only energy transfer is in a work form (i.e. there is no heat transfer to the perfectly insulated wall). Figure 15 elaborates on a cooling hole's temperature profile as the hole is centered at the nondimensional length of $\mathrm{x} / \mathrm{D}$ equal to zero ( $\mathrm{x}$ representing span distance over the $\mathrm{D}$, hole diameter). Moving further away vertically from the hole nondimensionally and downstream the temperature increases (approaching red). It's clear that the temperature along the wall $(\mathrm{y} / \mathrm{D}=0)$ remains at a lower temperature (noted as blue in the figure) until the eventual decay of the boundary layer effect due to the cooling hole.

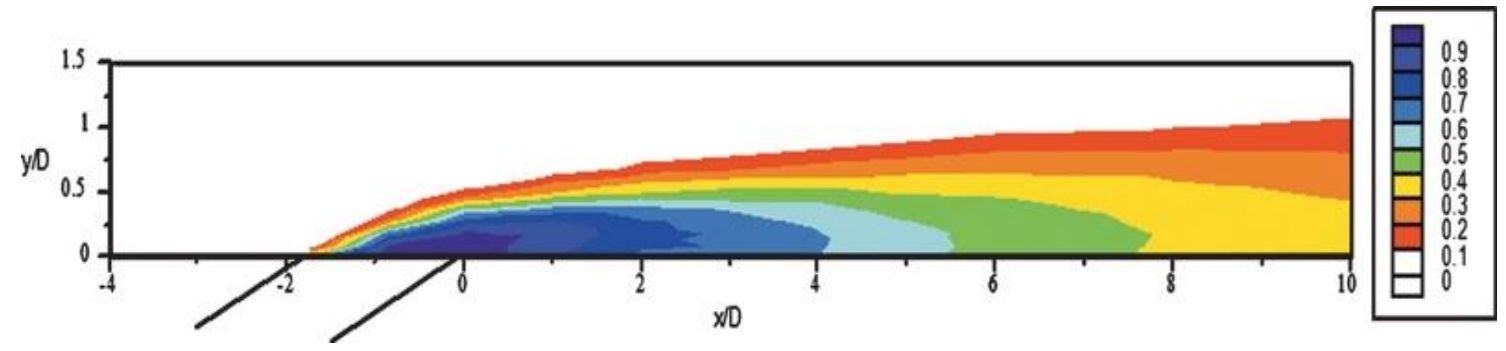

Figure 15 - Film Cooling Temperature Decay of Cooling Jet [19] 
This injected air may introduce higher turbulence in the boundary layer region thus increasing the local convective heat transfer coefficient in many, but not all, cooling scheme configurations. An ideal case of this cooling scheme would be to maintain attachment of the coolant to the surface of the airfoil and not shed into the freestream [13]. This idealization would maintain the surface airfoil blade temperature at the coolant temperature, therein minimizing the heat transfer to the blade wall. This, however, is not completely practical in demonstration and the diffusion of the coolant into the freestream is presented as in Figure 15 above.

\subsection{COOLING SCHEMES}

All the theory of the previous sections leads up toward the underlying goal of the present work of film cooling since both the freestream and boundary layer conditions greatly affect the cooling effectiveness of the turbine blade. While this investigation focuses on characterizing boundary layers to specifically understand just the fluid dynamic properties,

for the interest of expressing the purpose of the recommissioned tunnel, noting how cooling schemes utilize this fluid data and also incorporate temperature measurements is noted.

Introductory sections presented how commonly the turbine inlet temperature was a main critical factor in the thermal efficiency and therein the overall engine efficiency. Increases in the average turbine inlet temperature over the last 20 years have been roughly $8 \mathrm{~K}$ per year. Given that the general design rule many engine designers adhere to is that a $10 \mathrm{~K}$ rise in temperature will reduce the life of the component by $50 \%$, this trend of increasing inlet temperature and must be sufficiently accounted for. Figure 16 displays the progression of this increase in inlet temperatures and the spike of increase that results from the introduction of cooling methods around the mid-twentieth century [21]

INTRODUCTION 


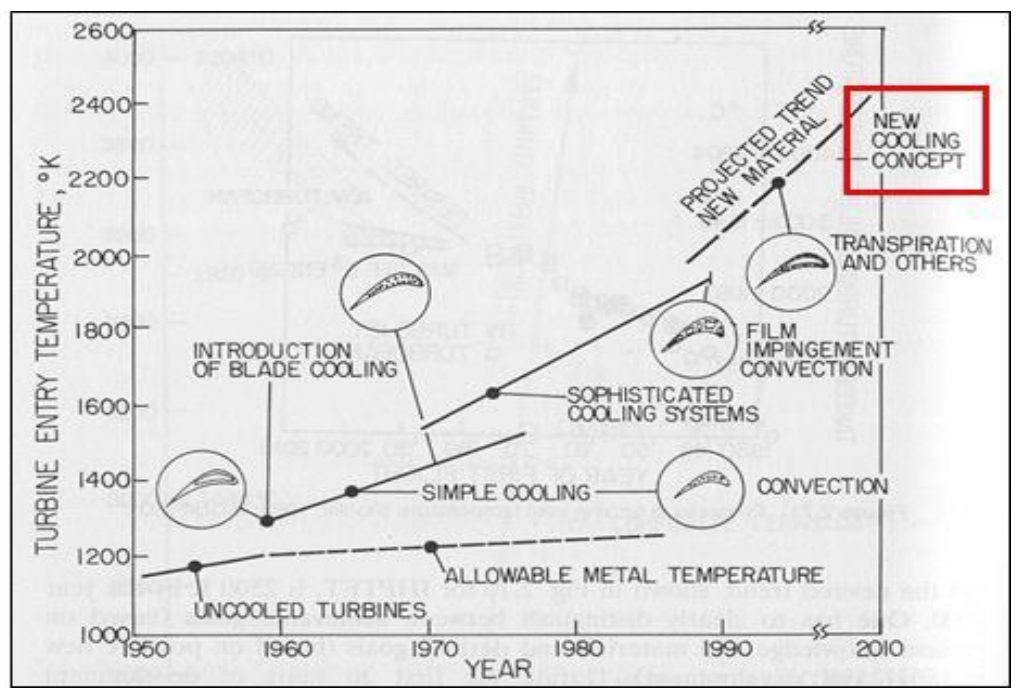

Figure 16 - Historical Progression of Turbine Inlet Temperatures [22]

A majority of experiments today non-dimensionally match conditions and enlarge the area of interest on turbine blades with the suction and pressure surfaces of turbine blades have convex and concave curvature, respectively. These investigations employ flat plate simulations and can accurately predict film cooling effectiveness. Since this review focuses primarily on characterizing the effect a cooling scheme has upon the boundary layer, introductory comments will be made discussing classical hole geometries such as straight hole geometries. More complex geometries such as angled holes and modern anti-vortex holes have shown improved cooling performance at low freestream turbulence intensities as compared to conventional straight holes [23].

Flat plate studies have investigated changing a variety of flow parameters including blowing ratio (BR), density ratio (DR), temperature ratio (TR), Reynolds number based on hole diameter, and the freestream turbulence intensity (Tu). In addition, investigations have evaluated cooling hole geometries and cooling hole parameters such as: non-dimensional values of hole pitch (P) and hole length (L) each non-dimensionalized by coolant injection angle $(\alpha)$ and hole diameter (D) to study their effects on adiabatic cooling effectiveness for a wall, $\eta_{a w}$, as previously expressed in Equation 1.1 . 
Previous research has concluded that the parameters of greatest importance when studying the variation of film cooling effectiveness were the freestream turbulence intensity, $\mathrm{BR}$, and the ratios of turbulence length scale to the hole diameter and the length scale to boundary layer thickness [24][25]. The blowing ratio (BR) is defined as the ratio of mass flow rate per unit area of coolant to the mass flow per unit area of freestream and is mathematically expressed as:

$$
B R=\frac{\rho_{c} U_{c}}{\rho_{\infty} U_{\infty}}
$$

As proposed by Han and Mechndale, the optimum blowing ratio for a straight hole geometry is roughly between $0.5-0.7$ for straight holes aligned in a straight row [26]. This range corresponds to blowing ratios achieved just before jet lift off and therein the detachment of a coolant jet from the blade surface. When jet-lift off occurs at higher blowing ratios, the coolant jets act like a solid cylinder in cross flow upon entering the freestream environment. It can be found that actual blowing ratios can exist on the order of 1.5 or even larger [9].

Other investigations have found additional issues that arise from trying to implement cooling films. One such occurs from a phenomenon named counter-rotating vortex pairs that form when downwash of these vortices draw down hot freestream gas and forces it upon the blade surface, subsequently pushing the coolant flow away from the surface due to upwashing [27]. As stated varying the blowing ratios, hole shapes, angles of hole inclination, lines of holes, all have been extensively studied and conclude that situationally cooling effectiveness can be increased with the variation of such parameters.

\subsection{FILM COOLING MEASUREMENT TECHNIQUES}

In order to measure film cooling effectiveness, there are numerous methods. While not used in this experimental investigation, mention of each is discussed in order to illuminate the motives of the presented experimental design. Four of these methods are designed to measure the surface temperature in a high spatial resolution. Such techniques are pressure 
sensitive paint, temperature sensitive paint, thermochromatic liquid crystals, infrared thermography, and thermocouples when used coherently with freestream and coolant temperature data [9].

\subsubsection{PRESSURE SENSITIVE PAINT (PSP)}

This method of measuring film cooling is nonintrusive and is utilized for steady-state and transient applications. This is an optical measurement method that consists of applying a luminescent coating that is oxygen permeable held together with a polymer binder which responds to localized wall pressure excitations. This response occurs when exposed to a short wavelength illumination source. This illumination source is typically a laser or light emitting diode (LED) which sends photons toward the painted surface. The captured local surface emission light intensity is inversely proportional to the region's surface pressure. Used in conjunction with a high-resolution specialized camera, the generated pixelated image essentially corresponds each pixel recorded as a pressure transducer by the paint luminescence sampling. Using intensity of reflected light, the coolant film effectiveness can be found after calibration, as well as the local velocity from pressure gradients. Since additional errors due to conduction are not present, the main purpose of this technique is film cooling [28].

\subsubsection{TEMPERATURE SENSITIVE PAINT (TSP)}

Quite similar to PSP, TSP utilizes luminescent molecules in a binder polymer instead reacting to the laser radiation from the LED by the process of thermal quenching. Thermal quenching allows the paint to reduce luminosity intensity as temperature increases corresponding to increases in local pressures or temperatures. This method operates with correspondence to the visible band of the electromagnetic spectrum, offering a large bandwidth. TSP only operates at an increasing temperature and is only operable for a single experiment needing reapplication for each subsequent experiment [29].

INTRODUCTION 


\subsubsection{THERMOCHROMATIC LIQUID CRYSTALS (TLC)}

These crystals are materials that change reflected color in the electromagnetic spectrum in response to temperature changes when illuminated by a white light. Each step change in temperature correlates to a hue and color change in the crystal yielding a transient observation of the film cooling effectiveness. A camera captures the light reflected and is then post-processed to reflect changes in temperature. These devices are available in a bandwidth of $0.1^{\circ} \mathrm{C}$ to $30^{\circ} \mathrm{C}$ [30]. This technique requires diligent cleaning of the substrate surface and normally a black underlayment for optimal results. Then after, the coating is applied in two suspensions encapsulated or capsulated. The encapsulated type is a liquid crystal bound in a roughly 10-micron sphere suspended in a water based binder material, whereas the capsulated is the material in a natural form and is susceptible to contamination. While operable at various camera angles, each repositioning requires recalibration.

\subsubsection{INFRARED (IR) THERMOGRAPHY}

Infrared thermography, like each other method previously discussed, is a nonintrusive measurement option, however, requires less substrate preparation. Utilizing an infrared camera, the transient emitted infrared energy from the object is recorded and subsequently converted into a temperature distribution map covering the area of the camera's viewing aperture. Operating in the thermal band of the electromagnetic spectrum, it can operate in any field without interference from other sources. This spectrum can be seen in Figure 17 below ranging from short to very long infrared wavelengths. IR cameras have a larger bandwidth than many of the other techniques with high accuracy and reliability when ideally calibrated.

INTRODUCTION 


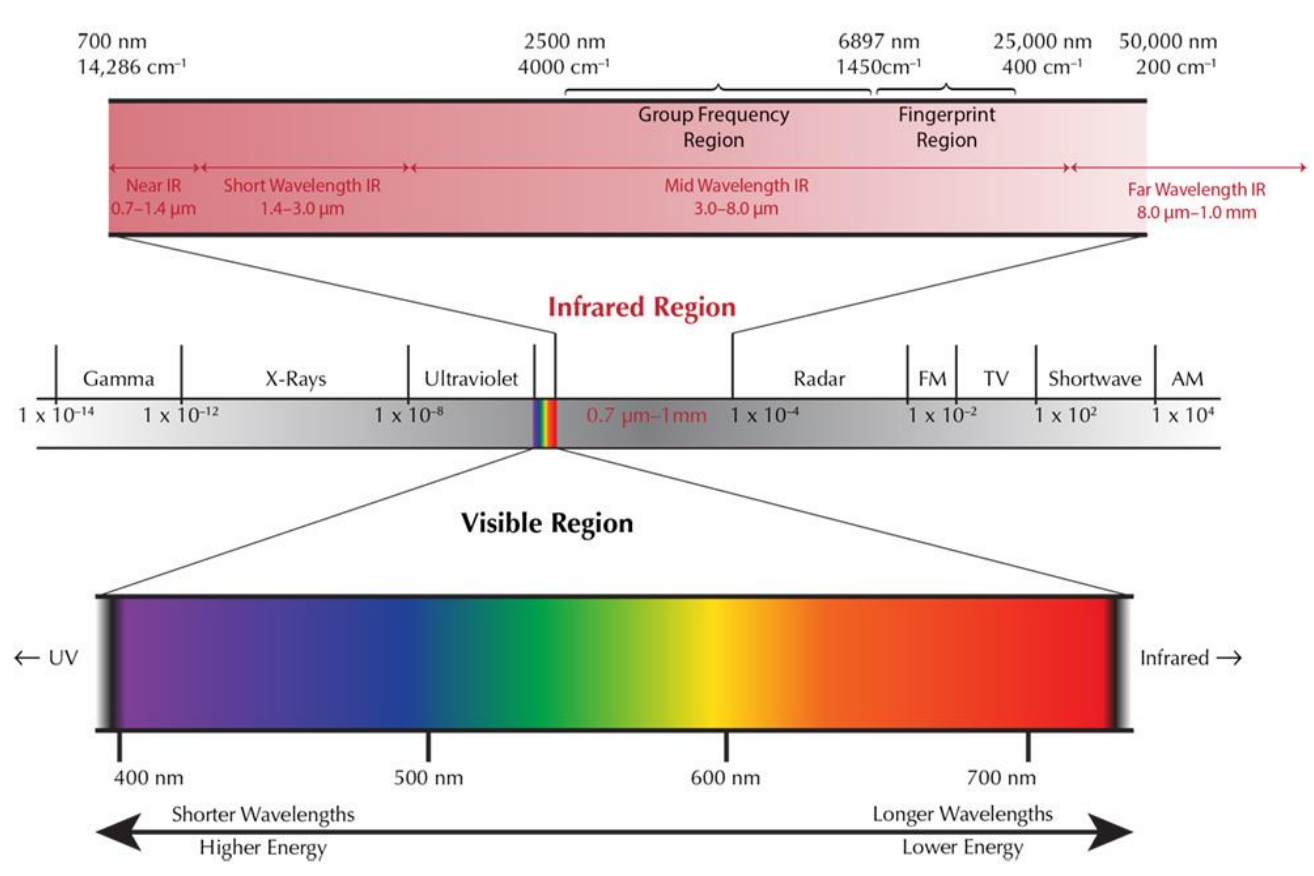

Figure 17 - Infrared Spectrum [31]

\subsubsection{THERMOCOUPLES}

Extensively used in film cooling testing as well as a variety of other applications, this intrusive method predates the other measurement techniques and yields less accuracy. Operating on the Seebeck effect which relates the voltage caused by a temperature difference on each side of a metal substrate, thermocouples output temperature readings depending on their discrete locations, mounted flush with the surface. Since each thermocouple only measures one location, dissimilar to the other techniques, this temperature measurement does not offer an overview of the entire operable test section. In order to do so would unreasonably require a large number of thermocouples covering the entire surface, which simply isn't practical, and degrades the quality of surface underrepresenting the end goal of testing.

\subsection{APPLICATIONS TO FUTURE WORK}

The above-mentioned film cooling measurement techniques are included to provide initial research on how this investigation's findings will establish a starting point for future cooling 
studies. The current facility is now capable of utilizing infrared thermography for film cooling studies as started at WVU by Hayes, et al., but never completed in the entirety, detail, and complexity as desired. Future work will include testing various cooling hole geometries with data acquired by a FLIR A655sc IR camera using a 45-degree lens. This camera provides a high spatial resolution and prevents interference or disturbances within the tunnel flow. Controlled via FLIR ResearchIR software in conjunction with Labview @ and Matlab@, surface temperature data will be collected above the cooling holes. Using IR thermography as well as hotwire data, future experimental investigations will be directly compared to Computations Fluid Dynamic (CFD) results for validation. 


\section{CHAPTER 2: BOUNDARY LAYERS \& FLOW CLASSIFICATION}

The following section details a mathematical derivation and expression for boundary layer equations stemming from the governing laws of fluid dynamics. This is provided in the carefully devised investigation progression to provide insight on what are the defining characteristics of a boundary layer in both laminar and turbulent states, but also to compare this experiments results to theoretical data (e.g. the Blasius solution). Furthermore, in addition to attempting to achieve a complete explanation of characteristics and theoretical representations, the following derivations are explained in order to also fundamentally explain the setup for how film cooling affects boundary layer energy and momentum transfer; which will be useful in future investigations by the author.

\subsection{GOVERNING EQUATIONS}

All fluid motion is governed by the fundamental laws of physics, namely the conservation of mass, the first and second laws of thermodynamics, and Newton's second law of motion. Since this investigation is concerned with experimental boundary layer work and not numerical CFD, the proceeding governing equations are mentioned, but not fully derived in order to achieve completeness in investigation discussion while maintaining brevity. The conservation of mass principle states that the time rate of change of a system's mass equals zero or expressed below mathematically in differential form and vector notation respectively:

$$
\begin{gathered}
\frac{\partial \rho}{\partial t}+\frac{\partial \rho u}{\partial x}+\frac{\partial \rho v}{\partial y}+\frac{\partial \rho w}{\partial z}=0 \\
\nabla \cdot V=0
\end{gathered}
$$

This expression is found in a control volume considering each direction of the coordinate plane and utilizing a Taylor series expansion as seen in Figure 18 below.

BOUNDARY LAYERS \& FLOW CLASSIFICATION 


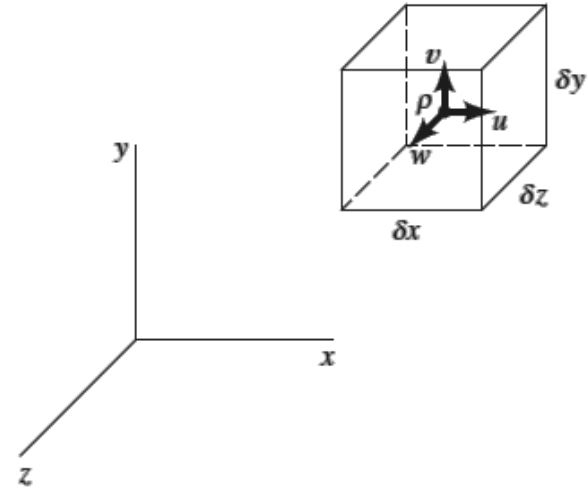

(a)

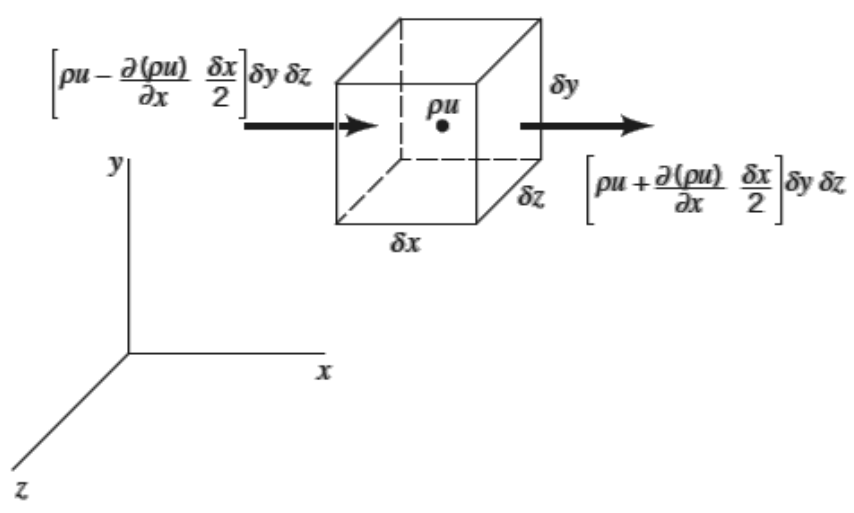

(b)

Figure 18 - Continuity Control Volume [32]

The continuity equation above expresses changes in the three spatial components of velocity with the $\mathrm{x}, \mathrm{y}$, and $\mathrm{z}$ directions (u,v,w respectfully) and the density $(\rho)$ with time when summed, must equal zero. In others words, the summation of the time rate of change of the mass in the control volume and the mass flow in and through the control surfaces must equal zero.

Equally important in understanding flow is the adaptation of Newton's second law where the time rate of change of the linear momentum of the system equals the sum of the external forces acting on the system. In a similar fashion to the continuity equation above, by performing a control volume analysis, as shown in Figure 19, the conservation of momentum can be derived and is applicable to any flow. 


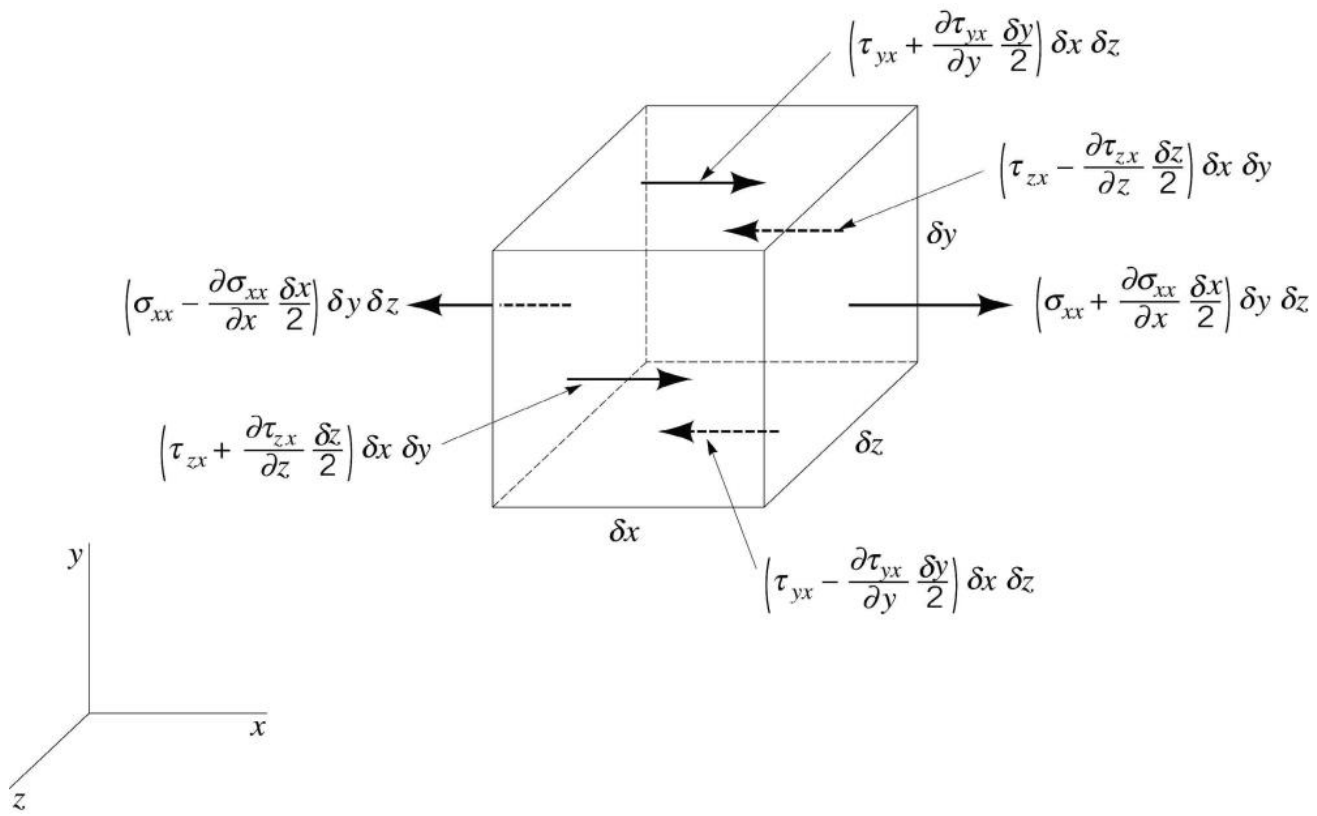

Figure 19: Conservation of Linear Momentum Control Volume [32]

The equation sums all the external forces in each spatial direction (e.g. body and surface) and equals to the rate of change of momentum yielding the following equations for $\mathrm{x}, \mathrm{y}$, and $\mathrm{z}$ directions respectfully:

$$
\begin{aligned}
& \rho g_{x}+\frac{\partial \sigma_{x x}}{\partial x}+\frac{\partial \tau_{y x}}{\partial y}+\frac{\partial \tau_{z x}}{\partial z}=\rho\left(\frac{\partial u}{\partial t}+u \frac{\partial u}{\partial x}+v \frac{\partial u}{\partial y}+w \frac{\partial u}{\partial z}\right) \\
& \rho g_{y}+\frac{\partial \sigma_{y y}}{\partial y}+\frac{\partial \tau_{x y}}{\partial x}+\frac{\partial \tau_{z y}}{\partial z}=\rho\left(\frac{\partial v}{\partial t}+u \frac{\partial v}{\partial x}+v \frac{\partial v}{\partial y}+w \frac{\partial v}{\partial z}\right) \\
& \rho g_{z}+\frac{\partial \sigma_{z z}}{\partial z}+\frac{\partial \tau_{x z}}{\partial x}+\frac{\partial \tau_{y z}}{\partial y}=\rho\left(\frac{\partial w}{\partial t}+u \frac{\partial w}{\partial x}+v \frac{\partial w}{\partial y}+w \frac{\partial w}{\partial z}\right)
\end{aligned}
$$

In these equations, the only body force considered is gravity, $\mathrm{g}, \sigma_{i, j}$ represents the normal forces and $\tau_{i, j}$ represents the shear forces of the $\mathrm{i}^{\text {th }}$ and $\mathrm{j}^{\text {th }}$ directions, and all other variables as defined in the continuity equations. In order to solve this set of nonlinear differential equations, simplifying assumptions must be made such as introducing the stress deformation relationships for a Newtonian fluid (not shown) and assuming incompressibility yielding the Navier-Stokes equations for the $\mathrm{x}, \mathrm{y}, \mathrm{z}$ directions respectively. 


$$
\begin{aligned}
& \rho\left(\frac{\partial u}{\partial t}+u \frac{\partial u}{\partial x}+v \frac{\partial u}{\partial y}+w \frac{\partial u}{\partial z}\right)=-\frac{\partial p}{\partial x}+\rho g_{x}+\mu\left(\frac{\partial^{2} u}{\partial x^{2}}+\frac{\partial^{2} u}{\partial y^{2}}+\frac{\partial^{2} u}{\partial z^{2}}\right) \\
& \rho\left(\frac{\partial u}{\partial t}+u \frac{\partial v}{\partial x}+v \frac{\partial v}{\partial y}+w \frac{\partial v}{\partial z}\right)=-\frac{\partial p}{\partial y}+\rho g_{y}+\mu\left(\frac{\partial^{2} v}{\partial x^{2}}+\frac{\partial^{2} v}{\partial y^{2}}+\frac{\partial^{2} v}{\partial z^{2}}\right) \\
& \rho\left(\frac{\partial u}{\partial t}+u \frac{\partial w}{\partial x}+v \frac{\partial w}{\partial y}+w \frac{\partial w}{\partial z}\right)=-\frac{\partial p}{\partial z}+\rho g_{z}+\mu\left(\frac{\partial^{2} w}{\partial x^{2}}+\frac{\partial^{2} w}{\partial y^{2}}+\frac{\partial^{2} w}{\partial z^{2}}\right)
\end{aligned}
$$

or written in vector notation as:

$$
\rho\left(\frac{D V}{D t}\right)=-\nabla p+\rho g+\mu \nabla^{2} V
$$

The Navier-Stokes equation utilizes the constitutive relations and introduces a pressure term, $\mathrm{p}$, from the normal stresses and the dynamic viscosity, $\mu$, from the shear stress relationships. Each equation (Continuity and Navier-Stokes) is general in form and is applicable to any continuum with an inertial reference frame. This equation, when used in conjunction with the continuity equation, provides a complete depiction of incompressible Newtonian fluid flow, and both apply to laminar and turbulent flows, the two classifications characterizing fluid motion.

With a thermodynamic perspective brought into view, a similar energy balance on the control volume yields for two-dimensional heat transfer with the control volume shown in Figure 20 below: 


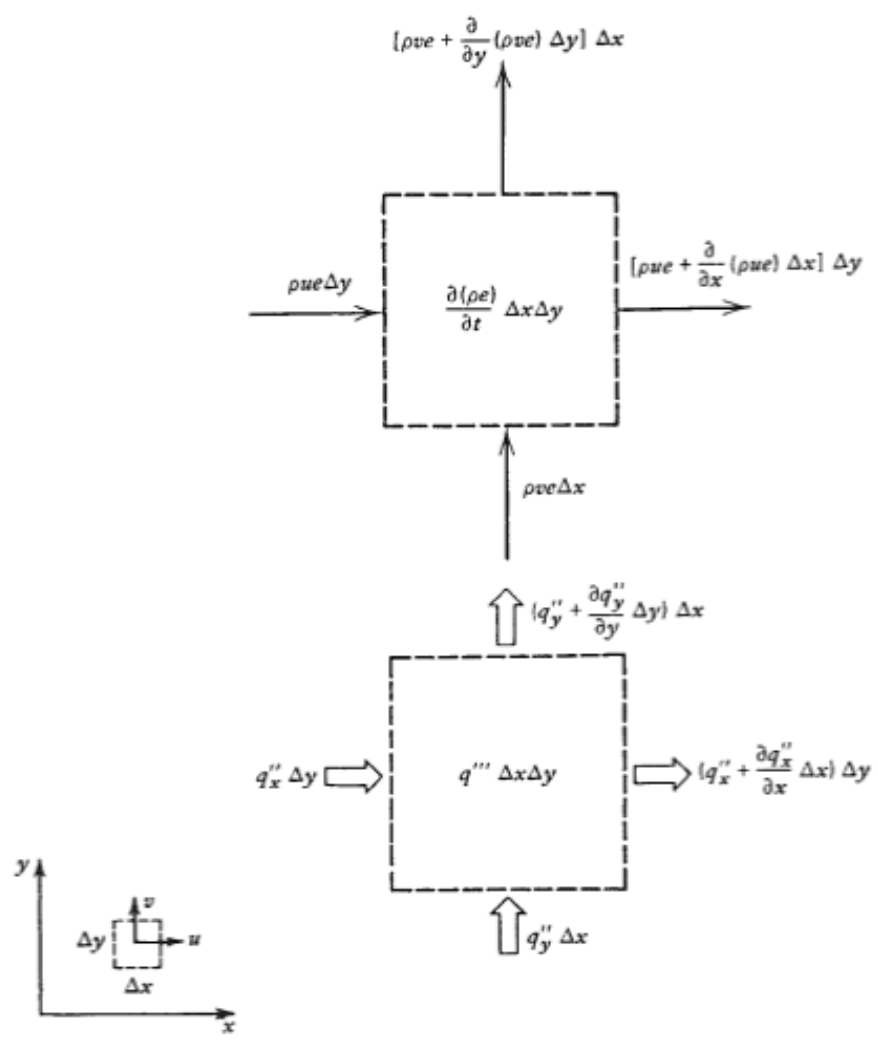

Figure 20 - Energy Equation Control Volume [33]

Thus yielding the energy balance equation:

$$
\frac{\partial \rho e}{\partial t}=-\left[\frac{\partial \rho u e}{\partial x}+\frac{\partial \rho v e}{\partial y}\right]-\left[\frac{\partial q^{\prime \prime} x}{\partial x}+\frac{\partial q^{\prime \prime} y}{\partial y}\right]+q^{\prime \prime}+\sigma_{x} \frac{\partial u}{\partial x}+\sigma_{y} \frac{\partial v}{\partial x}-\tau_{x y} \frac{\partial u}{\partial y}-\tau_{y x} \frac{\partial v}{\partial x}
$$

Likewise, when plugging in the constitutive equations to account for stresses, the continuity equation, and definition of enthalpy the equation written in vector notation is reduced to:

$$
\rho c_{p} \frac{D T}{D t}=\nabla \cdot(k \nabla T)+q^{\prime \prime}+\beta T \frac{D P}{D t}+\mu \Phi
$$

where $\rho$ is the density of the fluid, $c_{p}$ is the specific heat of the fluid, $\frac{D T}{D t}$ and $\frac{D P}{D t}$ are the substantial derivatives of temperature, $\mathrm{T}$, and pressure, $\mathrm{P}$, with respect to time, $\mathrm{t}$. $\nabla$ represents the gradient operator of the temperature field, $q^{\prime \prime}$ accounting for heat generation, $\beta$ is the 
coefficient of thermal expansion derived from Maxwell's relations, $\mu$ accounting for heat associated with the dynamic viscosity, and $\Phi$ containing the stresses which release heat due to the viscosity of the fluid, or viscous dissipation as defined here for incompressible fluids:

$$
\Phi=2 \mu\left[\left(\frac{\partial u}{\partial x}\right)^{2}+\left(\frac{\partial v}{\partial y}\right)^{2}\right]+\left(\left(\frac{\partial u}{\partial x}\right)+\left(\frac{\partial v}{\partial y}\right)\right)^{2}
$$

When further assumptions are employed such as constant thermal conductivity, negligible viscous dissipation, and no heat generation, the equation drastically simplifies to:

$$
\rho c_{p} \frac{D T}{D t}=\mathrm{k} \nabla^{2}(T)
$$

Fluids flows are classified into two categories: laminar or turbulent. Laminar flow is a flow that is undisturbed by any disruptions in the flow field and is much easier to study than turbulent flows. This is due to the fact that laminar flows behave more predictably and have less randomization of fluid motion to describe. The Navier-Stokes equation in turbulent flow as mentioned is valid; however, the randomness in respect to time that occurs for each velocity components adds complexity making an analytical solution unavailable. A muchstudied phenomenon, turbulence is investigated out of necessity to truly understand a complete picture of fluid flow. As a flow encounters more instability downstream from the initial point of contact, the laminar attributes of the flow being to transition to turbulent. This transition point is generally expressed in terms of the nondimensional flow parameter called the Reynolds number as defined below.

$$
R e_{l}=\frac{\rho V l}{\mu}
$$

The Reynolds number is physically interpreted as the ratio of inertial forces to viscous forces found from the Buckingham Pi theorem. As the velocity, V, becomes larger, the Reynolds number also increases where the viscous forces are eventually unable to dampen disturbances caused by the inertial forces. The results are instability, the growth of velocity fluctuations, and tumbling vortex motions in three-dimensional Cartesian space. 
Additionally, vortex shedding off the immersed object develops vortex stretching leading to a vast range of turbulent eddies. For freestream flow, turbulence is defined as $\operatorname{Re}>5 \times 10^{5}$.

\subsubsection{TRANSITION TO TURBULENCE}

Turbulent flows, due to their inherent chaotic behavior, are much harder to fully define than laminar flows. As most flows occurring in nature are inherently turbulent, such as the conditions experienced in a gas turbine compressor or turbine, further knowledge is required to properly account for and predict control volume outlet conditions. To completely define turbulence though is rather difficult in one brief statement. According to Mathieu and Scott, there are 11 defining attributes that most completely conceptualize turbulent flows [34].

1. Turbulence is/appears to be stochastic or chaotic in both the time and space domains. Though there is structure to eddies in the flow, the Navier-Stokes equations which govern all incompressible Newtonian flow are deterministic; there is a sensitive dependence on initial conditions yielding a clear average behavior but irreproducible in detail.

2. Occurs always in three-dimensional space. Without such consideration, dissipation scales and stretching of vortices is not considered.

3. Vorticular development of a large scale range of fluctuating vorticity in random generation.

4. Dissipative with energy when at the smallest eddy scales (Kolmogorov length scale $\left(\eta_{k}\right)$ in the form of heat through a cascading effect.

5. Obeys the Navier-Stokes Laws, however, is unlikely to be comprehensively predicted by a solution.

6. Non-linear phenomena due to the convective acceleration term Navier Stokes equations and leading to nonlinear Reynolds Stress development.

7. High Reynolds number phenomena resulting from instability at higher Re values.

BOUNDARY LAYERS \& FLOW CLASSIFICATION 
8. Continuum Phenomena where the smallest eddy scale is determined at the Kolmogorov scale greater than the mean free path. The Kolmogorov scales tend toward being homogenous, isotropic, and universal as Reynolds number is increased.

9. Large ranges of length and time scales for eddies that become independent of Reynolds number for large Reynolds numbers. At these high Reynolds numbers, large eddies become almost inviscid.

10. Behavior is often intermittent.

11. When concerning mass transfer, turbulence is a diffusive phenomenon.

Once the general characteristics that define turbulence are detailed, further elaboration into the types of turbulence is necessary. Dewan details that there are three basic simplifications that allow further study of turbulence categorizing into three types [35].

1. Homogeneous turbulence. Is defined where the same behavior is experienced in all parts of the flow.

2. Isotropic turbulence. In the case that the statistical characteristics of the flow be the same in all directions.

3. Stationary turbulence. Exists when the time average of all flow properties do not vary with time.

However, in practicality, no actual turbulent flow is perfectly homogenous or isotropic. While a flow region may macroscopically be laminar and stable, when examining infinitesimal distances from the surface, miniscule perturbations are experienced only dampened out by viscous effects.

Although numerical simulations are valid options for modeling turbulent flow, most research has utilized the concept of time averaging. By applying time averaging to the equations of motion, the Reynolds equations are found, as is done similarly for the energy equation.

BOUNDARY LAYERS \& FLOW CLASSIFICATION 


$$
\bar{Q}=\frac{1}{T} \int_{t_{0}}^{t_{o}+T} Q d t
$$

The expression above is valid for any variable $\mathrm{Q}$ resolved into a mean value. Turbulence parameters are expressed as a combination of this mean value, $\bar{Q}$, and a fluctuations term $Q^{\prime}$. Considering incompressible turbulent flow with constant transport properties with respective fluctuation terms are listed in Table 1.

Table 1 - Time-Averaged and Fluctuation Terms

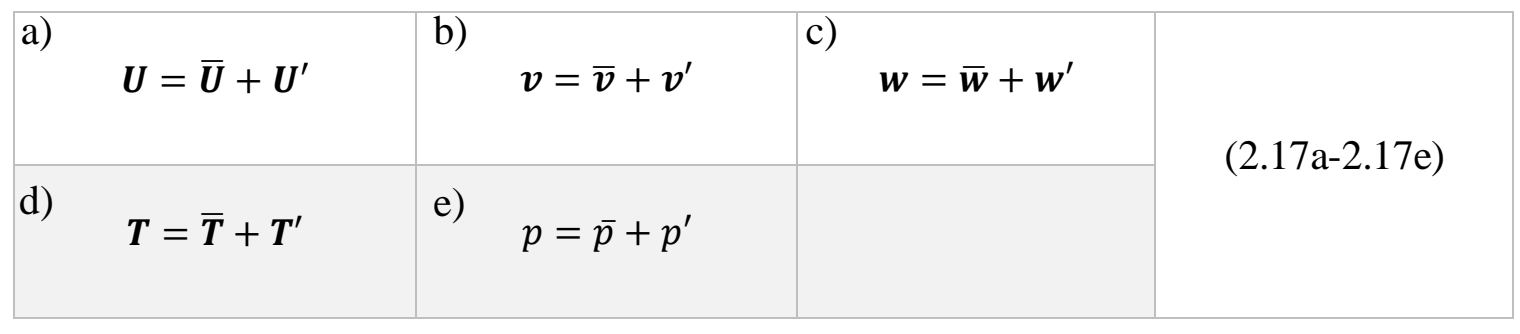

Plugging these relations of averaging and fluctuations into the continuity and Navier Stokes equations (respectively listed), the turbulence of the flow has been dealt with, and the equations each still satisfy the underlying laws of physics.

$$
\begin{gathered}
\frac{\partial \bar{u}}{\partial x}+\frac{\partial \bar{v}}{\partial y}+\frac{\partial \bar{w}}{\partial z}=0 \\
\frac{\partial u \prime}{\partial x}+\frac{\partial v^{\prime}}{\partial y}+\frac{\partial w \prime}{\partial z}=0 \\
\rho\left(\frac{D \bar{V}}{D t}\right)+\rho \frac{\partial}{\partial x_{j}}\left(\overline{u_{\jmath}^{\prime} u_{\jmath}^{\prime}}\right)=-\nabla p+\rho g+\mu \nabla^{2} \bar{V}
\end{gathered}
$$

Both the fluctuation and time-averaged continuity equations are valid except for varying density, which would result in additional coupled terms. The turbulent momentum equation is complicated by the introduction of the turbulent inertia tensor, $\overline{u_{\jmath}{ }^{\prime} u_{\jmath}{ }^{\prime}}$, which cannot be neglected. Rearranging the Navier Stokes equations to reflect the turbulent inertia terms as if they were stresses, yields slightly more insight into how these terms behave if the total stress on the system were composed of Newtonian viscous stresses plus the turbulent stress tensor. This simplification is shown as follows: 


$$
\rho\left(\frac{D \bar{V}}{D t}\right)=-\nabla p+\rho g+\nabla \cdot \tau_{i j}
$$

where

$$
\tau_{i j}=\mu\left(\frac{\partial u_{j}}{\partial x_{j}}+\frac{\partial v_{j}}{\partial y_{j}}\right)-\rho \overline{u_{\jmath}^{\prime} u_{\jmath}^{\prime}}
$$

The time-averaged energy equation for incompressible flow with constant properties then takes the form of:

$$
\rho c_{p} \frac{D \bar{T}}{D t}=-\frac{\partial}{\partial x_{i}} q_{i}+\bar{\Phi}
$$

where the viscous dissipation term transforms to:

$$
\bar{\Phi}=\frac{\mu}{2} \overline{\left(\frac{\partial \bar{u}_{l}}{\partial x_{l}}+\frac{\partial u_{\imath}{ }^{\prime}}{\partial x_{\imath}}+\frac{\partial \bar{u}_{J}}{\partial x_{J}}+\frac{\partial u_{J}^{\prime}}{\partial x_{J}}\right)^{2}}
$$

and a new term, $\mathrm{q}_{\mathrm{i}}$, is introduced reflecting a collection of conduction and turbulent convection terms (shown respectively below) into a total heat flux vector:

$$
q_{i}=-k \frac{\partial \bar{T}}{\partial x_{i}}+\rho c_{p} \overline{u_{\imath}^{\prime} T^{\prime}}
$$

Given as Mathieu and Scott propose the example of an infinitely long circular cylinder submerged inside a uniform, steady flow perpendicular to the axis of the cylinder, one can see how the development of turbulence initiates for external flows as shown in Figure 21 below. 


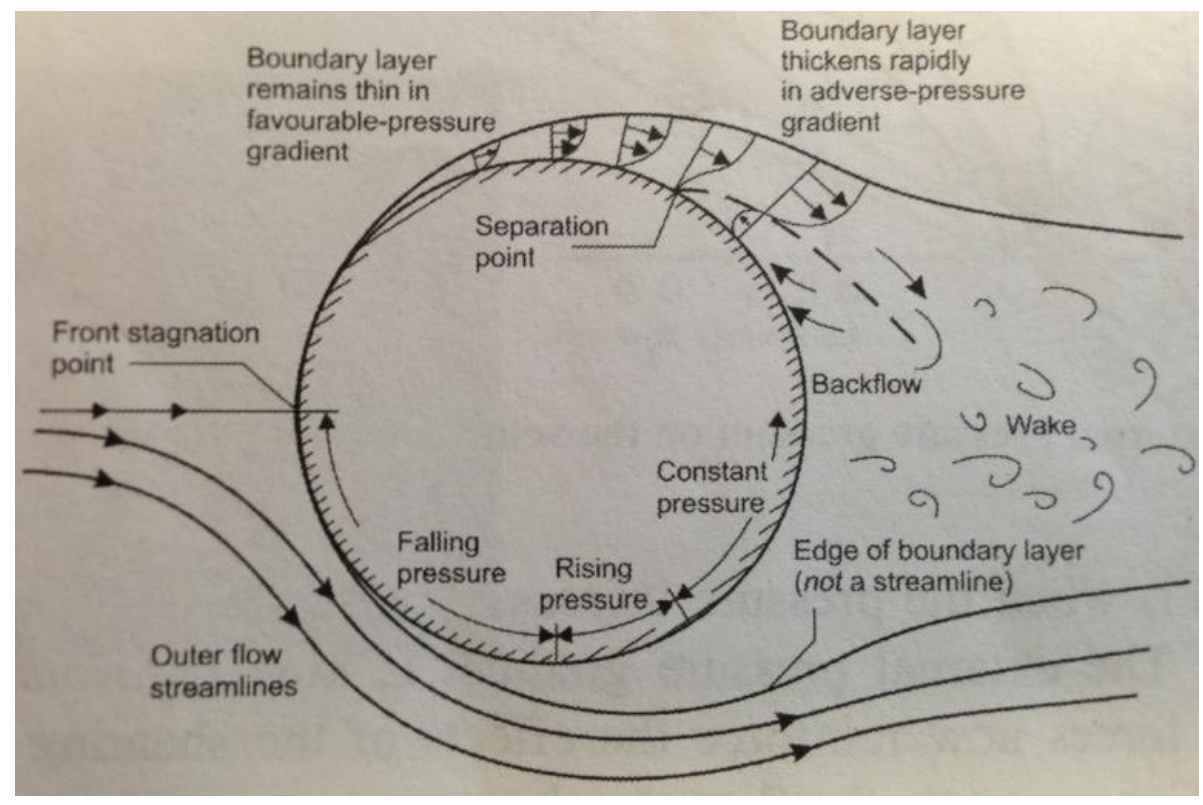

Figure 21 - Cylinder Boundary Layer Turbulent Separation [36]

As the flow progresses around the object, boundary layers near the cylinder surface emerge, stretching from the stagnation point at the leading edge of the cylinder to symmetrically positioned separation points on the cylinder sides. It is from these points that shear layers result in boundary layer separation generating a wake region where circulating eddies exist and continuously feed the vorticity. Once the critical Reynolds number value is reached, the flow becomes completely unstable even within infinitesimal perturbations [34]. The vortices previously mentioned and that are shed off as shown in Figure 22 below constitute a large driver of turbulence caused by the existence of the body in the flow. These vortices are shed within the wake in time-periodic wavelike oscillations. Up through the levels of very high Reynolds numbers, the region of turbulence gradually moves closer to the cylinder until the entire wake becomes turbulent. The flow is then aperiodic yet still retains a cyclical vortex shedding behavior. Outside the wake region, the flow is still characterized by a laminar state as it has been unaffected by the effects of the blunt body's presence. 


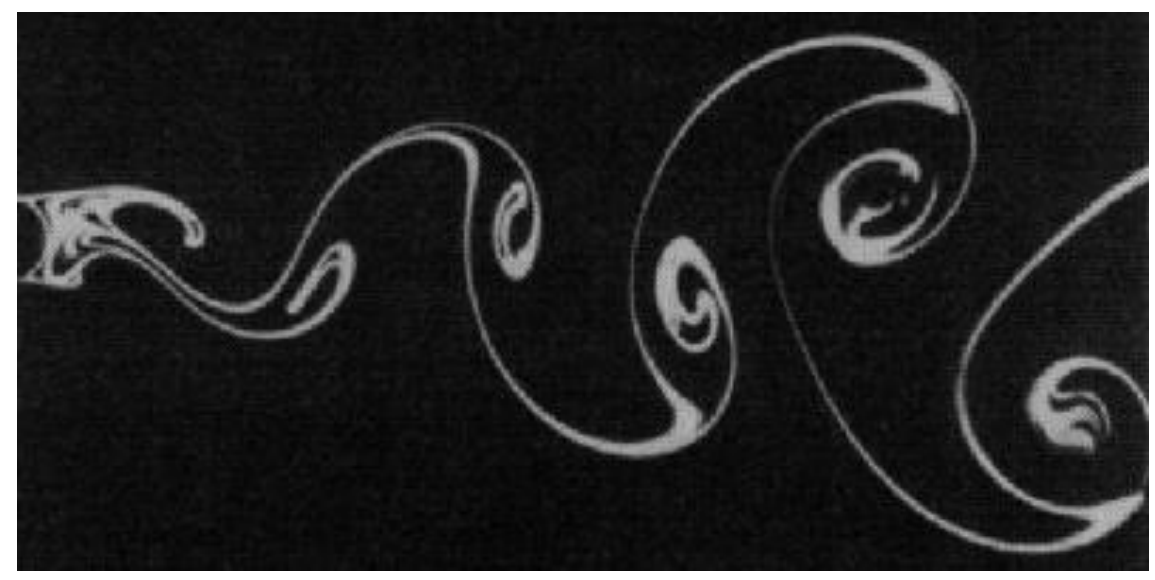

Figure 22 - Von Karmen Vortex Shedding [37]

These vortices can be disastrous as notoriously epitomized by the Tacoma Narrows Bridge disaster; where the vortex shedding frequency matched the natural frequency of the bride thus causing failure and catastrophe.

As detailed the cause of turbulence in the wake of an object is caused by boundary layer separation from the object's surface. With that, boundary layer characterization is crucial in identifying how long the flow will remain "attached" to the surface which affects everything from drag, pressure, and in the case of a turbine blade, heat transfer design.

\subsection{BOUNDARY LAYER IMPORTANCE}

When considering any flow, the area of most interest is where parameters are changing. This area is near the surface, such as the blade of a turbine, in the region called the boundary layer, and is defined to be a very thin region with a nearly constant pressure in the direction normal to the surface. It is the region of the flow where viscous effects are important such that the velocity profile develops from the stream-wise zero point $(x=0)$ to the end of the entrance length where the velocity profile no longer varies. The boundary layer is a theoretical region that allows researchers an approximation method of simplifying the partial differential governing equations of conservation of mass, energy, and momentum as there does not exist a general analytical or uniqueness theorem to solve these equations [38]. As 
previously stated, this region is where the external cooling schemes affect the performance and efficiency of the gas turbine engine itself. Film cooling in particular involves knowing the state of the boundary layer in order to appropriately estimate the heat transfer to the blade. When the flow is turbulent an increase in heat transfer occurs providing better insulation on the turbine blade.

\subsubsection{LAMINAR BOUNDARY LAYERS}

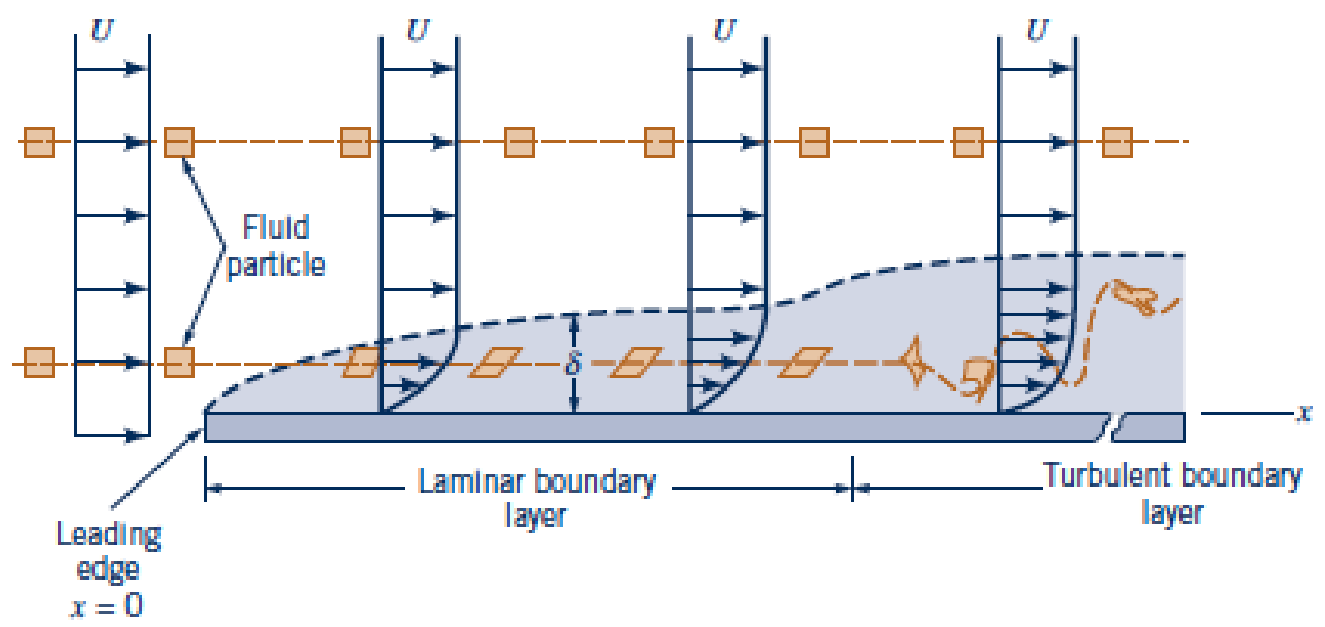

Figure 23 - Distortion of Fluid Particle Entering Boundary Layer [32]

The concept of a boundary layer can be better explained from a Lagrangian flow field description i.e. tracking a particle as it enters a boundary layer from the freestream flow, as visualized in Figure 23. The particle maintains shape with no rotation in the freestream flow. The flow outside the boundary layer is said to be irrotational; while once a fluid particle enters the boundary layer, the particle obtains a nonzero vorticity. Upon entering the boundary layer, it begins to distort due to the velocity gradient imposed by a velocity in freestream and the no-slip condition imposed at the surface. The no-slip condition is the imposed boundary condition that is necessary for solving the momentum equation of motion. Boundary layer characteristics chiefly depend on: both the state of the freestream flow and the distance downstream from initial flow contact. Standard for laminar flow boundary layers, at some location downstream of the flow along the surface of the immersed body, the 
flow will eventually trip to the turbulent state as the fluid particles become distorted due to irregular and random motion associated with a disruption in the flow. This transition is due to the onset of instability characterized by the Reynolds Number. Separation of the boundary layers occurs when the flow quickly decelerates, i.e. when the outer pressure gradient is positive or increasing, referred to as an adverse pressure gradient. Due to viscous effects, not all the pressure field is converted to kinetic energy leading to the flow reversing around the separation point. Classical linear theory which uses a parallel flow approximation predicts instability increasing with streamwise distance measured by the thickness or displacement thickness of the boundary layer. Boundary layer thickness, $\delta$, is defined as the distance, $y$, from the immersed surface where the local velocity, u, reaches $99 \%$ of the free-stream velocity, U. Therefore, making this the first important description of a boundary layer.

$$
\delta_{.99}=y \text { where } u=.99 U
$$

The reason $99 \%$ of freestream is chosen is an arbitrary value set by the definition of the boundary layer displacement thickness. The displacement thickness represents the amount that the submerged body thickness must be increased so that the fabricated uniform inviscid flow has the same flow properties as an actual flow as defined below:

$$
\delta^{*}=\int_{0}^{\infty}\left(1-\frac{u}{U}\right) d y
$$

where $\delta^{*}$ denotes the displacement thickness as the integral of one minus the ratio of local velocity and the freestream velocity. This allows for conceptualizing the displacement of streamlines the viscous effects on the plate cause. Additionally, another boundary layer thickness, known as the boundary layer momentum thickness, $\Theta$, is used when the drag of an immersed body is desired. This value represents the distance where the boundary layer should be displaced to compensate for reduction in the momentum of the fluid due to the formation of the boundary layer. The mathematical representation for such is expressed as:

$$
\Theta=\int_{0}^{\infty} \frac{u}{U}\left(1-\frac{u}{U}\right) d y
$$


A term called the shape factor, $\mathrm{H}$, is expressed as the ratio of the momentum thickness to the displacement thickness and is useful in boundary layer analysis:

$$
H=\frac{\delta^{*}}{\Theta}
$$

The previous three boundary characteristics are used in boundary layer analyses in association with another, discussed later, defining the thermal properties of the boundary layer region; all of which definitions are provided under the assumption that the boundary layer is thin.

Having now defined the three boundary layer thicknesses associated with fluid motion, it is important to now express the boundary layers mathematically in terms of the general equations of fluid motion for the cases of laminar and turbulent states. Detailed previously as the continuity and the Navier-Stokes equations, these laws hold true for boundary layer regimes yet are currently impossible to solve solely analytically. However, using the approximations as introduced and employing various substitutions, the boundary layer equation for momentum and energy as derived from the previously noted momentum and energy equations respectively for two-dimensional flow are:

$$
\begin{gathered}
\left(u \frac{\partial u}{\partial x}+v \frac{\partial u}{\partial y}\right)=-\rho \frac{\partial p}{\partial x}+\rho g_{x}+v\left(\frac{\partial^{2} u}{\partial y^{2}}\right) \\
\left(u \frac{\partial T}{\partial x}+v \frac{\partial T}{\partial y}\right)=\alpha \frac{\partial^{2} T}{\partial y^{2}}
\end{gathered}
$$

Completing a simple scale analysis we can further redefine the boundary layer thicknesses and introduce the thermal boundary layer thickness, $\delta_{T}$, to complete the description of both the momentum and thermal properties of the region near the surface wall. Considering the variables on the scales of:

$$
x \sim L \quad: \quad y \sim \delta \quad: \quad u \sim U_{\infty}
$$

inertial, pressure, and friction terms are rewritten respectively as: 


$$
U_{\infty} \frac{U_{\infty}}{L}, v \frac{U_{\infty}}{\delta}: \frac{P}{\rho L} \quad: \quad v \frac{U_{\infty}}{L^{2}}, \frac{U_{\infty}}{\delta^{2}}
$$

thus allowing for rearranging the boundary layer thicknesses, wall shear stress, skin coefficient, thermal boundary layer thickness, and heat transfer coefficient in a more convenient non-dimensional form as respectively shown in Table 2.

Table 2 : Boundary Layer Relations

\section{Momentum Relations:}

\begin{tabular}{|l|l|l|l|}
\hline & b) & c) \\
\hline$\frac{\delta}{\boldsymbol{x}}=\boldsymbol{a}_{1} \boldsymbol{R} \boldsymbol{e}_{x}^{-1 / 2}$ & $\tau=\rho U_{\infty}^{2} R e_{x}^{-1 / 2}$ & $C_{f}=\frac{\tau}{\frac{1}{2} \rho U_{\infty}^{2}}=a_{2} R e_{x}^{-\frac{1}{2}}$ & $(2.34 \mathrm{a}-2.34 \mathrm{c})$
\end{tabular}

The coefficients $a_{1,2}$ are significant when looking at the various methods of derivation via either the similarity solution where the constants equal one, or the integral solutions where a control volume is used for derivation and the constants equal $\mathrm{a}_{1,2}=4.640,0.646$ respectively. In the case of the exact solution through the similarity derivation the variables $\mathrm{a}_{1,2}$ are 4.92, 0.664 respectively. These equations are generally mostly accepted at lower Reynolds numbers, since at higher values randomization increases and deviate from correct solutions.

As previously stated this section seeks to establish a path for detailing how a boundary layer and its characteristics are defined. While each term is not specifically used in great detail for the purpose of this report. It was the authors intend to provide this analytical description in order to completely understand boundary layer theory and establish a guide for an experimental description. This outline will also serve useful for future work detailing film cooling and influences of boundary layer cooling holes. Therefore, the purpose of Table 2 achieves this snapshot summary of defining boundary layer parameters and their definitions. For the simplified case of flow over a flat plate, as this investigation is simplified 
down to, the defining characteristics for laminar flow is given by the Blasius solution later detailed and compared to turbulent boundary layer approximations given in Table 3.

\subsubsection{TURBULENT BOUNDARY LAYERS}

The differences between the laminar and turbulent states vary in both regards to momentum and heat transfer. Increased momentum transport due to turbulence from the free stream flow to the flow near the wall makes turbulent boundary layers more resistant to flow separation. The turbulent flow does enhance mixing which can be beneficial or detrimental depending on the boundary layer thickness. There is a higher momentum deficit in a turbulent boundary layer. As with the cylinder example, if it should occur that a boundary layer is laminar, there is insufficient momentum exchange occurring, making the flow unable to adjust to the increasing pressure thereby inducing separation from the surface. Likewise, when considering the case where the flow is turbulent, the increased transport of momentum due to Reynolds stresses, as previously noted from the free-stream flow toward the wall, adds to the momentum stream-wise within the boundary layer allowing the flow to overcome the adverse pressure gradient. Even still, the flow eventually does separate nevertheless, but further downstream [39].

Additionally, unlike laminar boundary layer flow, the various properties (mass, momentum, energy) are also transported across the boundary layer by the turbulent eddies. Since there is not a precise expression for turbulent shear stress, solutions are not available for turbulent flow analytically. The preferred modeling right now is with computational fluid dynamics.

Similar to boundary layers as freestream conditions the integral momentum, energy, and other relations for turbulent flow can utilize the time averaging approach and use continuity to simplify unnecessary cluttering terms. The integral momentum equation for turbulent momentum boundary layers containing momentum, displacement thicknesses, and shape factor are respectively: 


$$
\begin{gathered}
\frac{d \theta}{d x}+(2+H)\left(\frac{\theta}{U_{3}}\right)\left(\frac{d U_{e}}{d x}\right)=\frac{\tau_{w}}{\rho U_{e}^{2}}=\frac{C_{f}}{2} \\
\Theta=\int_{0}^{\infty} \frac{\bar{u}}{U_{e}}\left(1-\frac{\bar{u}}{U_{e}}\right) d y \\
\delta^{*}=\int_{0}^{\infty}\left(1-\frac{\bar{u}}{U_{e}}\right) d y \\
H=\frac{\delta^{*}}{\Theta}
\end{gathered}
$$

This form proposed by Karman (1921) is similar to the laminar flow relation; however, the three thicknesses cannot be related as easily with one term as in the laminar equation [38]. The shear stresses take on an additional time averaging solution form where the random velocity components that constitute this momentum transfer (shear force) are $u$ ' and $v^{\prime}$ for the $\mathrm{x}$ and $\mathrm{y}$ directions respectively.

$$
\tau=\mu \frac{d \bar{u}}{d y}-\rho \overline{u^{\prime} v^{\prime}}=\tau_{\text {lam }}+\tau_{\text {turb }}
$$

Clearly, the shear stress is the addition of the turbulent term to the previously known expression for laminar flow. This turbulent component is always found to be positive, always yielding a greater stress for turbulent flow than laminar, as fundamentally makes sense. In the boundary layer, the dominant term is the turbulent term, except very near the wall, ideally named the turbulent shear.

\subsection{BLASIUS AND TURBULENT APPROXIMATIONS}

Using the expressions listed above for various cases, simplifications can be made to allow easier estimation of these boundary layer defining parameters. Listed below in Table 3 are simplifications of mentioned equations for the case of flow across a smooth, flat plate aligned parallel to a uniform stream. Its noted that laminar values are exact for this particular case and are listed to three significant digits. These were first derived by Blasius and are dubbed the Blasius solution. These equations are only valid for laminar flow subjected to the 
assumptions made in the Blasius Solution. The Blasius Solution stems from introducing a similarity variable, $\eta$, defined as:

$$
\eta=y \sqrt{\frac{U}{2 v x}}
$$

where $\mathrm{y}$ is the surface normal direction, $\mathrm{U}$ is the freestream velocity, $v$ is the kinematic viscosity, and $\mathrm{x}$ is the stream-wise distance from the flow entry point. By additional substitution with the stream function given as:

$$
\psi \sqrt{2 v U x} f(\eta)
$$

where the variable $f(\eta)$ is a function that is later determined. After considerable manipulation and rearrangement, the boundary layer momentum equation takes the form:

$$
f^{\prime \prime \prime}+f f^{\prime \prime}=0
$$

then further solving with the boundary conditions of this third order equation as follows:

$$
f^{\prime}(0)=f(0)=0 \text { and } f^{\prime}(\infty)=1
$$

Accompanying the Blasius Solution in Table 3 are Power Law approximations that more closely modify the laminar Blasius Solution to account for turbulence in the flow more closely aligning with empirical data trends. The power laws displayed involve increasing the power of the root containing the Reynolds number. Though not used in the investigation, the now nonzero normal wall velocity could also be expressed in the positive direction, developing a "blowing" into the freestream. This blowing is characterized in terms of film cooling by the blowing ratio as previously defined in Equation 1.1. This type of boundary layer manipulation has been previously and will be used in the future by the author for cooling hole analysis, further justifying the need for this numerical explanation and description. 
Table 3 - Boundary Layer Approximations

\begin{tabular}{|l|c|c|c|c|}
\hline Property & \multicolumn{1}{|c|}{$\begin{array}{c}\text { Laminar } \\
\text { (a) }\end{array}$} & $\begin{array}{c}\text { Turbulent via } \\
\mathbf{1} / 7 \text { Power Law } \\
\text { (b) }\end{array}$ & $\begin{array}{c}\text { Turbulent via } \\
\mathbf{1} / 5 \text { Power Law } \\
\text { (c) }\end{array}$ & $\mid$ \\
\hline $\begin{array}{l}\text { Boundary Layer } \\
\text { Thickness }\end{array}$ & $\frac{\delta}{x}=\frac{4.91}{\sqrt{R e_{x}}}$ & $\frac{\delta}{x} \cong \frac{0.16}{\sqrt[7]{R e_{x}}}$ & $\frac{\delta}{x} \cong \frac{0.38}{\sqrt[5]{R e_{x}}}$ & $(2.43 \mathrm{a}-2.43 \mathrm{c})$ \\
\hline $\begin{array}{l}\text { Displacement } \\
\text { Thickness }\end{array}$ & $\frac{\delta^{*}}{x}=\frac{1.72}{\sqrt{R e_{x}}}$ & $\frac{\delta^{*}}{x} \cong \frac{0.02}{\sqrt[7]{R e_{x}}}$ & $\frac{\delta^{*}}{x} \cong \frac{0.48}{\sqrt[5]{R e_{x}}}$ & $(2.44 \mathrm{a}-2.44 \mathrm{c})$ \\
\hline $\begin{array}{l}\text { Momentum } \\
\text { Thickness }\end{array}$ & $\frac{\theta}{x}=\frac{.664}{\sqrt{R e_{x}}}$ & $\frac{\theta}{x} \cong \frac{0.016}{\sqrt[7]{R e_{x}}}$ & $\frac{\theta}{x} \cong \frac{0.37}{\sqrt[5]{R e_{x}}}$ & $(2.45 \mathrm{a}-2.45 \mathrm{c})$ \\
\hline $\begin{array}{l}\text { Local Skin } \\
\text { Friction } \\
\text { Coefficient }\end{array}$ & $C_{f, x}=\frac{.664}{\sqrt{R e_{x}}}$ & $C_{f, x} \cong \frac{0.016}{\sqrt[7]{R e_{x}}}$ & $C_{f, x} \cong \frac{0.059}{\sqrt[5]{R e_{x}}}$ & $(2.46 \mathrm{a}-2.46 \mathrm{c})$ \\
\hline
\end{tabular}

The turbulent equations approximations are expressed via a common empirical approximation for the time-averaged velocity profile called the one-seventh-power and onefifth-power laws. Turbulent values however due to large uncertainty associated with turbulence in general, are listed to only two significant digits.

Again, while attempting to not be over repetitive, the purpose of Tables 2 and 3 are to provide the derivation and summary of defining boundary layer equations. This sets a good theoretical datum for the author in future boundary layer investigations, where detail will be required to completely define flow parameters. While presented with exhaustible detail, this information is not crucial to this investigation's proceedings, but does offer significance into how the boundary layer is mathematically defined and utilized for this experiment, therefore deemed pertinent to include. 


\subsection{BOUNDARY LAYER SUCTION}

As incorporated in this study, boundary layer suction is used as a means of controlling the boundary layer height. Continuing the progression of mathematical derivations, by modifying the previously introduced Blasius Solution equation from the previous section, the case to nonzero wall velocity is recognized. In this case, the boundary conditions just need to change in order to reflect the change to a nonzero value as follows:

$$
f^{\prime}(0)=0 \text { and } f(0) \neq 0 \text { and } f^{\prime}(\infty)=1
$$

The streamwise wall velocity is still zero from the no-slip condition, and other assumptions previously made are still valid. Shown previously in Table 3 are the Blasius Solution equations used later for theoretical comparison with experimental results.

As the boundary layer encloses an area of lower momentum than the rest of the freestream flow, adding suction is a means of drawing out this low momentum fluid therein reducing the height of the boundary layer. The suction develops the velocity profile in a strong negative curvature (favorable pressure gradient), and delays turbulence transition due to its stability. Ultimately this simply means adding a negative surface normal velocity to account for. The amount of suction can be adjusted via suction fan speed setting to directly correlate with the amount of air drawn out of the boundary layer. Literature conducting similar testing on film-cooling effectiveness have published boundary layer thicknesses nondimensionalized by the cooling hole diameter $(\delta / D)$. Table 4 below details previously published boundary layer thicknesses and respective sources.

BOUNDARY LAYERS \& FLOW CLASSIFICATION 
Table 4 - Non-dimensionalized Boundary Layer Thicknesses

\begin{tabular}{|c|c|}
\hline Author & $\boldsymbol{\delta} / \boldsymbol{D}$ \\
\hline Yuen [40] & 1 \\
\hline Sung Jung [41] & 1.5 \\
\hline Meador [42] & 0.56 \\
\hline Lutum [43] & 0.45 \\
\hline Gritsch [44] & 0.5 \\
\hline
\end{tabular}

The cooling hole diameter for the current facility setup at WVU is 0.5 inches, so it was decided that a value between 0.5 inch to 1.0 inch for $\delta / D$ would be a target of desired boundary layer thickness. Data collected by Whitlow, et al. prior to the author recommissioning the facility at WVU will be used for comparison and for further validation of this investigation's findings as detailed in the proceeding experimental description section [45].

Detail regarding how this suction manipulation of the boundary layer is utilized in this investigation are as follows. Results from trials of no suction ("open") and "full" suction are compared and analyzed. "Full" suction refers to the fact that the fan used to draw air from within the boundary layer is no constricted in any way, as for instance monitored with a value. Further details explaining setup and configuration of experimental operation are later expressed in proceeding sections.

\subsection{MEASURING TURBULENCE}

As previously noted turbulence is an important phenomenon in fluid flow contributing significantly to the mass, momentum, and energy transport. In addition, turbulence also plays a significant role in fluid friction losses and induced noise generation. There are three primary experimental methods of turbulence measurement: constant temperature hotwire anemometry (CTA), laser-Doppler anemometry (LDA), and particle-imaging velocimetry 
(PIV). One of the most accepted and useful methods of quantifying turbulence is the method of hotwire anemometry, as this investigation employs.

\subsubsection{HOTWIRE ANEMOMETRY}

Hotwire anemometry operates on the working principle of the cooling effect of a flow across a heated body, in this case, a cylinder. Utilizing a Wheatstone bridge and servo amplifier, the sensor is maintained at a constant resistance, and hence constant temperature, fed by a varying current. This current runs through a probe support to a 5-millimeter-long tungsten filament several microns thick at the end of the probe. Following the laws of convection heat transfer, as a velocity blows across the probe, the bridge voltage is increased in order to maintain that temperature. The corresponding voltage therefore required to maintain this temperature is directly correlated to the velocity of the flow the probe experiences. The system is unique in that it can support high temporal resolution of data sensitive to fluctuations up to several hundred kilohertz $(\mathrm{kHz})$ without sacrificing spatial resolution capable of measuring eddies down to $1 \mathrm{~mm}$ or less.

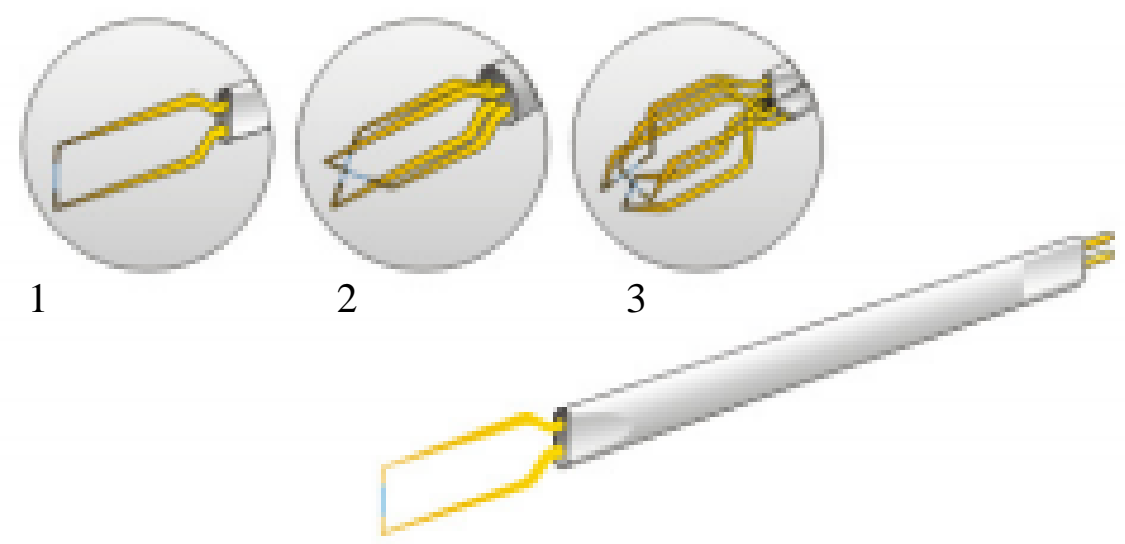

Figure 24 - CTA Probe [46]

Figure 24 above shows the various probe heads that can be used in hotwire applications. The single wire probe as shown in circle 1, top left, of Figure 23, is the probe used in this investigation. This probe is capable of examining a one-dimensional, uni-directional flow 
mounting the probe perpendicular and the prongs holding the filament parallel to the flow. Probes depicted in Figure 23 circles 2 and 3 are for measuring two-dimensional flows; selection of which is determined from the onward orientation of the velocity vector. These more complex probes require advanced software data signal processing units in order to handle the presented unique flow conditions (e.g. fully reversed flow).

The combination of low thermal inertia and high gain enable this significant response time to flow fluctuations. Outfitted with a variety of orientation and number of probe configurations, the sensor can simultaneously record velocity components in each spatial direction. This method does require calibration and correction for temperature sensitivity.

\subsubsection{LASER DOPPLER ANEMOMETRY}

Unlike CTA, LDA is a nonintrusive optical measurement technique. This method records a high spatial resolution, with increments distances anywhere from centimeters to meters in each spatial dimension. This is coupled with a high temporal resolution which permits instantaneous time averaged information. LDA utilizes a laser, beam splitter, transmitting/receiving optics component, and a photo detector to operate this technique. A vibrating piezoelectric crystal is attached generating acoustical waves acting similar to an optical grid. A Bragg cell, used as the beam splitter, outputs two beams focused into optical fibers delivering to a probe containing transmitting/receiving optics. In the probe, the beams from the fibers exit parallel, and are focused by a lens where the beams intersect inside a measurement volume. The light intensity is modulated due to interference between the beams, producing places of high light "fringes". The velocity can then be extracted by the light scattered by tiny "seeding" particles pumped into the flow field carried by the fluid as it moves downstream. A receiving lens collects the scattered light and focuses it on a photo detector, producing a signal at the Doppler frequency. Using the fringe distance and the Doppler frequency the velocity value is achieved. Doppler bursts are filtered and amplified using Fast Fourier Transform (FFT) [47]. Figure 25 shows the setup schematic for an LDA system.

BOUNDARY LAYERS \& FLOW CLASSIFICATION 


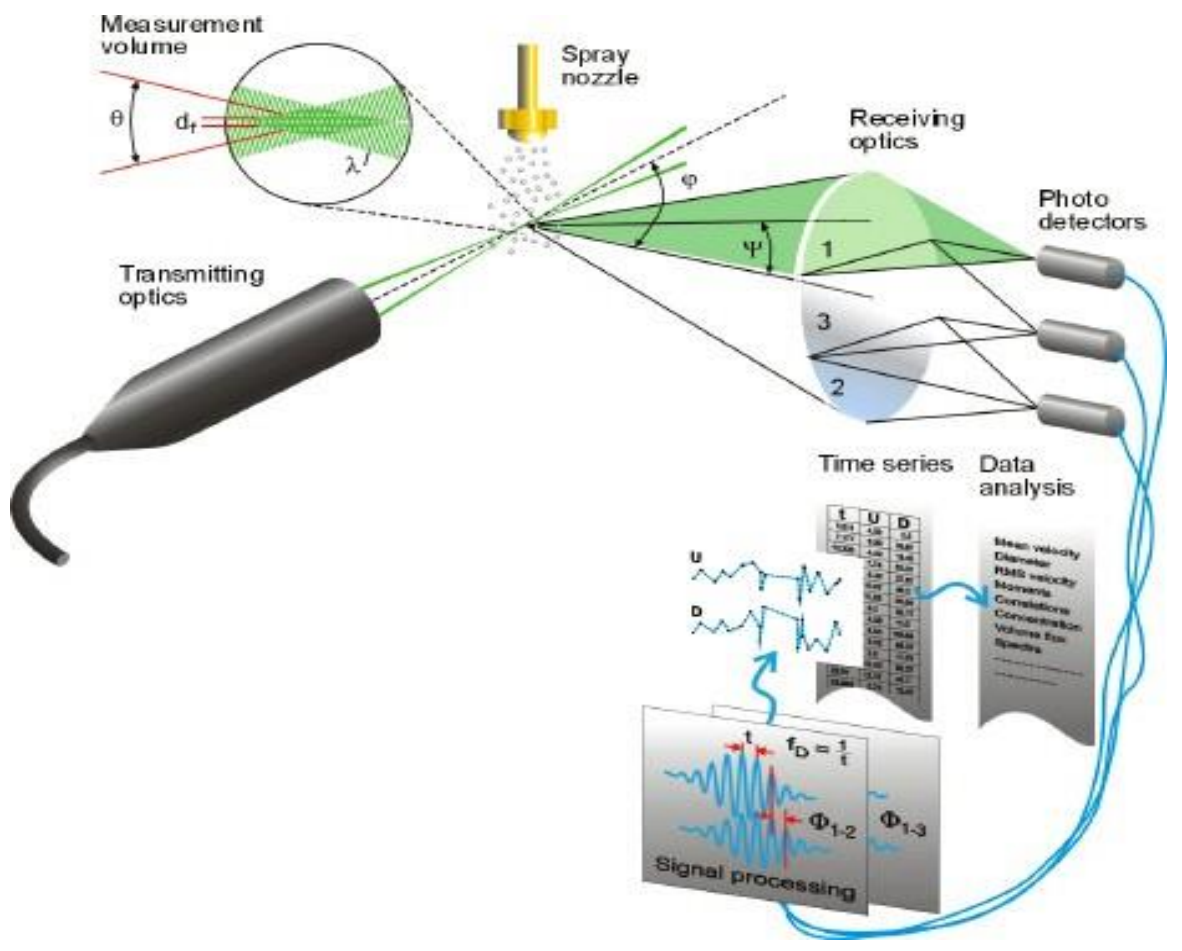

Figure 25 - LDA Setup [47]

The seeding particles are primarily used only in gas flow where they need to be large enough to scatter light but small enough not to disturb the characteristics of any object in the flow. Typical size range is around 1 micron to 10 microns either in solid or droplet forms. This method does not require calibration; however, a frequency shift correction is applied to account for particle flow reversals.

\subsubsection{PARTICLE IMAGE VELOCIMETRY}

This is a whole flow field measurement technique which provides instantaneous velocity vector measurements in a planar field. This method measures only two velocity components; however, it can record all three spatial dimensions with the use of a stereoscopic approach. Similar to LDA, this method is nonintrusive and measures the velocities of micro-sized particles seeded in the flow. Limited only by the spatial resolution of an imagining camera, this option yields high resolution in both spatial and temporal dimensions. Deriving the velocity vectors from subsections of a focused cross-section measuring the distances 
instantaneously and dividing by a known interval of time. A camera captures an illuminated seeded particle from two short duration laser light pulses noting the time between each pulse. The images are dividing into regions called interrogation areas where each image frame is cross-correlated with each other. This correlation yields a signal peak where average particle displacements are found through sub-pixel interpolation. Dividing this displacement by the pulse time between the lasers produces a velocity.

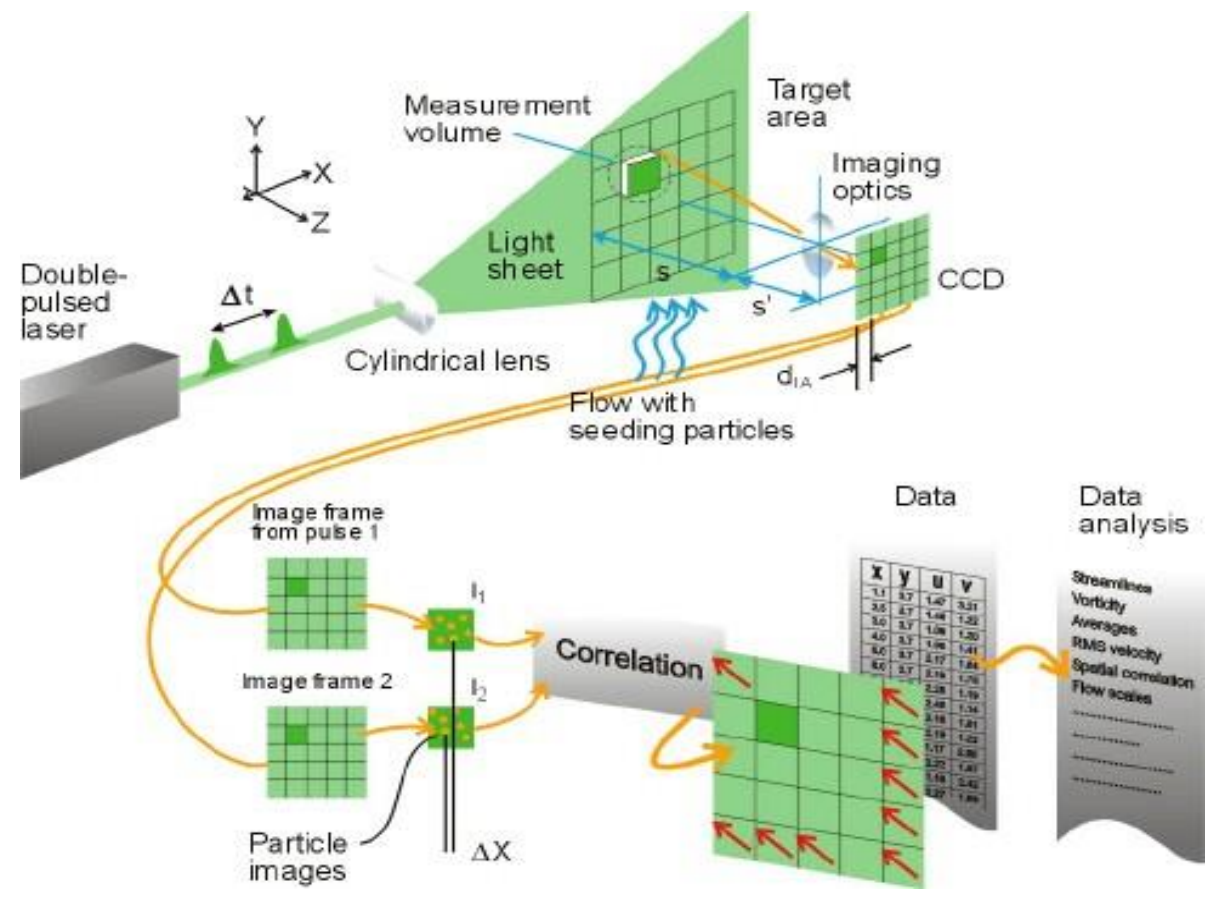

Figure 26 - PIV Setup [48]

Repeating this methodology for multiple interrogation windows gives a whole flow vector field of velocity. Results of the practice are similar to CFD simulations of large eddy calculations and velocity maps [48]. Figure 26 details how PIV system would process data.

\subsection{PROCESSING MEASURED TURBULENCE}

When processing turbulence data from any of the discussed methods, the flows must be first expressed in terms of mean values $(\bar{U})$ as shown in Figure 27 below with superimposed fluctuations $\left(U^{\prime}\right)$. White details that there are two methods of turbulent-flow analysis: one 
using a statistical theory of turbulent correlation functions and the other a semiempirical turbulent modeling of mean quantities [38]. The first method utilizes the frequency, oscillations, and other statistical interactions with each other. The second employs more engineering relevance with mean velocity, temperatures, wall friction, and heat transfer, shear thicknesses and root-mean-square fluctuations.

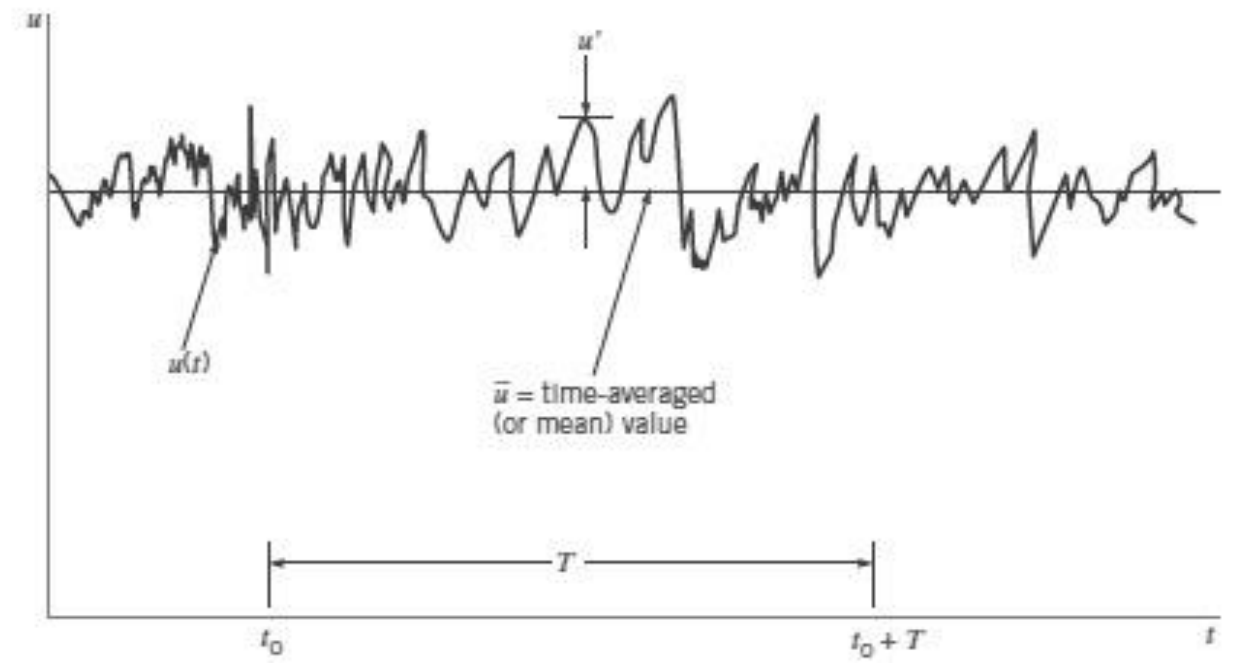

Figure 27 - Time-averaged and fluctuating velocity of turbulent flow [32]

The equation for a measured value of velocity at a given sample time, $\tilde{u}$, can be expressed as a summation of the mean velocity, $\bar{U}$, and the fluctuating velocity component, $u^{\prime}$ as follows [49]:

$$
\tilde{u}=\bar{U}+u^{\prime}
$$

The time mean value for velocity similar to the equation previously discussed is represented by the expression:

$$
\bar{U}=\frac{1}{T} \int_{t_{0}}^{t_{o}+T} U(x, y, z, t) d t
$$

with the time interval, $\mathrm{T}$, representing a considerably longer time than that of the longest fluctuation period, but shorter than an average velocity unsteadiness. The fluctuating part of 
the velocity is the time-varying part that varies from the average value as previously expressed in Section 2.1.1 where the time average of the fluctuations is zero. Assuming an evenly distributed range of fluctuations on either side of the average, and realizing that the square value of the fluctuation mean cannot be zero as expressed in Figure 28 below, the level of turbulence or turbulence intensity can be found.

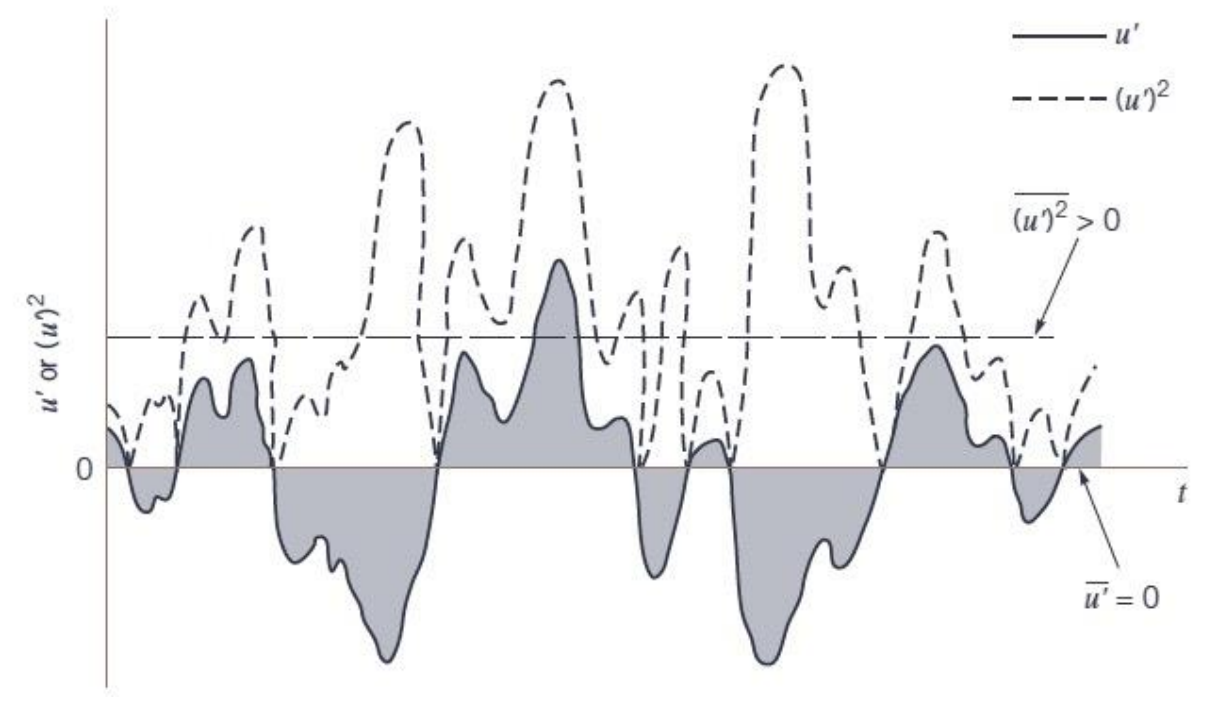

Figure 28 - Average of Fluctuations and Average Square of Fluctuations Imposed on Signal [32]

The turbulence intensity varies from one flow situation to another and can be large in a strong headwind or smaller in a lighter yet still turbulent breeze. This value quantifies this scenario mathematically as the root mean square of the average of the fluctuation squared over the average velocity:

$$
T u=\frac{\sqrt[2]{\overline{\left(U^{\prime}\right)^{2}}}}{\bar{U}}=\frac{U_{r m s}^{\prime}}{\bar{U}}
$$

This equation can reflect both the time and frequency domains. The larger the turbulence intensity value is contributed to the larger fluctuations in velocity. This value for the investigation attempted to be controlled, as is commonly done, by the use of a passive bar grid design placed upstream of the test section later described. 
As noted, the value of $U_{r m s}^{\prime}$, is the root mean square (RMS) for the fluctuating velocity signal can also be determined in the frequency domain using the power spectral density (PSD) for the fluctuating velocity component. The PSD yields an exemplification of the energy at each frequency of turbulent fluctuation, also known as the eddy scale. Integrating this spectrum would yield the fluctuating energy. Utilizing a fast Fourier transform (FFT), the fluctuating velocity component of the signal is decomposed, and the RMS value can be found thus providing the turbulence intensity.

The turbulent eddy time-scale is an additional term used to characterize turbulence. This turbulent integral length scale is found based on the peak to peak distance of the measured maximum turbulence intensity using an autocorrelation technique. The autocorrelation $\left(\mathrm{R}_{11}\right)$ of the fluctuating velocity component can be used to calculate the integral time scale $(T)$ by integrating under the curve to the first zero crossing as the lag time $(\tau)$ goes to zero, the RMS value of the velocity signal squared yields the unnormalized value of the autocorrelation.

The integral turbulence length scale is found by using the integral time scale, $T_{m}$, found from time integral of the autocorrelation curve of the fluctuating part of the velocity, $R_{11}$ as shown in Equation 2.50 below.

$$
R_{11}(\tau)=\frac{\overline{u(t) \cdot u(t+\tau)}}{u^{\prime 2}}=\frac{1}{N}\left(\frac{\sum_{1}^{N} u_{i} \cdot u_{i+j}}{u^{\prime 2}}\right)
$$

where $\tau$ is the total autocorrelation lag time equal to the product of the number of total time steps, $\mathrm{j}$, with the time step, $\Delta \tau$, going toward zero.

$$
T_{m}=\int_{0}^{\infty} R_{11}(\tau) d \tau=\sum_{i}^{N_{0}} R_{11} \Delta \tau
$$

Expressed in integral form first, the expression for the time integral scale can be approximated in summation notation until the first zero crossing, $N_{0}$, via the right-hand side of the above equation. Summation is only to the first zero crossing due to noise that exists in the auto-correlation. Using this value the integral length scale, $\Lambda_{x}$, representative of the 
largest eddies in the turbulent flow field, is found from the product of the integral time scale and the mean velocity as shown:

$$
\Lambda_{x}=\overline{\bar{U}} * T_{m}
$$

Equation 2.53 is representative of Taylor's hypothesis of frozen turbulence, expressing $\Lambda_{x}$ to represent the distance between the largest subsequent turbulence eddies corresponding to recorded "hits" of the data acquisition system as vortices are generated.

BOUNDARY LAYERS \& FLOW CLASSIFICATION 


\section{CHAPTER 3: EXPERIMENTAL SETUP}

\subsection{EXPERIMENTAL FACILITY}

The goal of this study is to characterize boundary layer parameters in both laminar and turbulent situations and then compare to additional experimental trials where a boundary layer suction fan acting as an active suction system is implemented and utilized. As stated the fan will draw out low momentum fluid from the surface and is expected to decrease the boundary layer height along with other effects to boundary layer parameters. In order to accomplish this, an experimental facility was recommissioned at West Virginia University (WVU). The wind tunnel is a subsonic, low temperature, continuous flow, open loop setup. This facility was previously utilized for blade cooling schemes and had fallen into a need of update and relocation. Detail of the refabrication, reassembly, modifications, and updates to this facility are discussed in the proceeding sections as well as the tunnel component makeup.

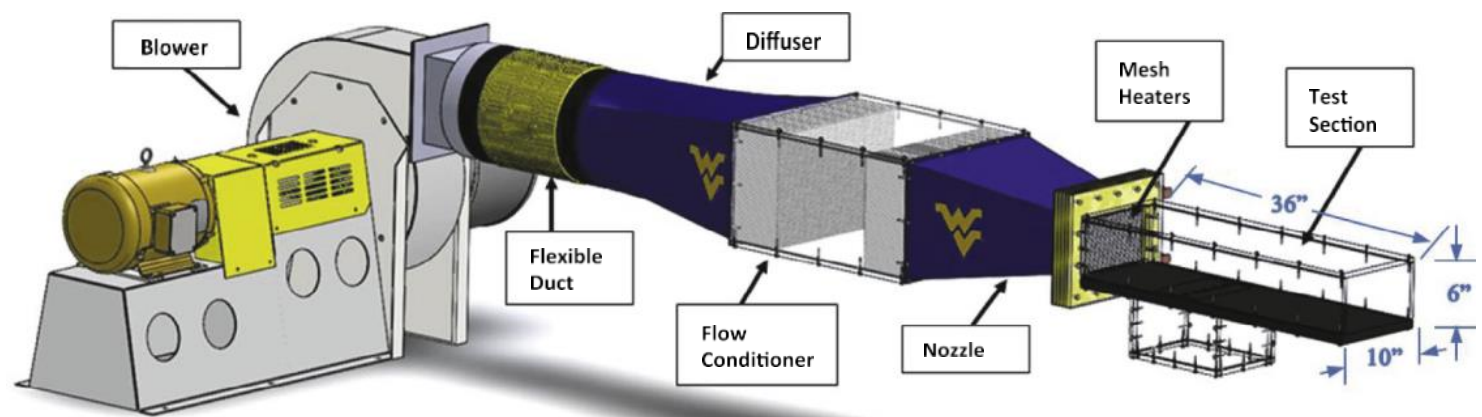

Figure 29 - WVU Wind Tunnel

\subsubsection{FLOW GENERATION \& CONDITIONING}

Shown in Figures 29 above and 30, 31 below is the experimental film cooling facility where the investigated boundary measurements were completed. Originally commissioned and completed by Hayes et al., it was designed to non-dimensionally simulate the aerothermal environment typically experienced within the first vane stage of a gas turbine engine [23]. Due to the low curvature nature of actual turbine blades, flat plate models 
accurately represent cooling holes manufactured in turbine blades while allowing easier data acquisition [23].

The flow is driven by a Cincinnati 7.5 HP radial blade exhauster fan on a direct drive motor configuration. This fan's intake is outfitted with a fine paper screen to prevent dirt and debris particles from entering the freestream flow. Controlling the fan is a Bardac Optidrive E2 controller providing the operator variety in testing RPMs and therefore a range of test section velocities. Loaded to capacity, the fan/motor combination is capable of reaching 3625 RPM thereby yielding a test section freestream velocity of approximately $25 \mathrm{~m} / \mathrm{s}$. A flexible duct at the radial fan exit is a flexible duct used to dampen out any vibrations within the flow.

Next, a diffuser with an expansion ratio of 2:1 is used to slow the flow speed, and aims at developing a uniform cross-sectional velocity. The expansion ratio of the diffuser is also designed at such an angle in the attempt to mitigate boundary layer separation [50] while converting the flow from a circular cross-section to a larger rectangular cross-section; important for the downstream stages. The diffuser leads into a flow straightener with plastic Plascore honeycomb sections on either side in order to develop the most controlled laminar flow possible before introducing controlled turbulence. This comb structure reduces swirl, while preparing the flow to enter the nozzle section.

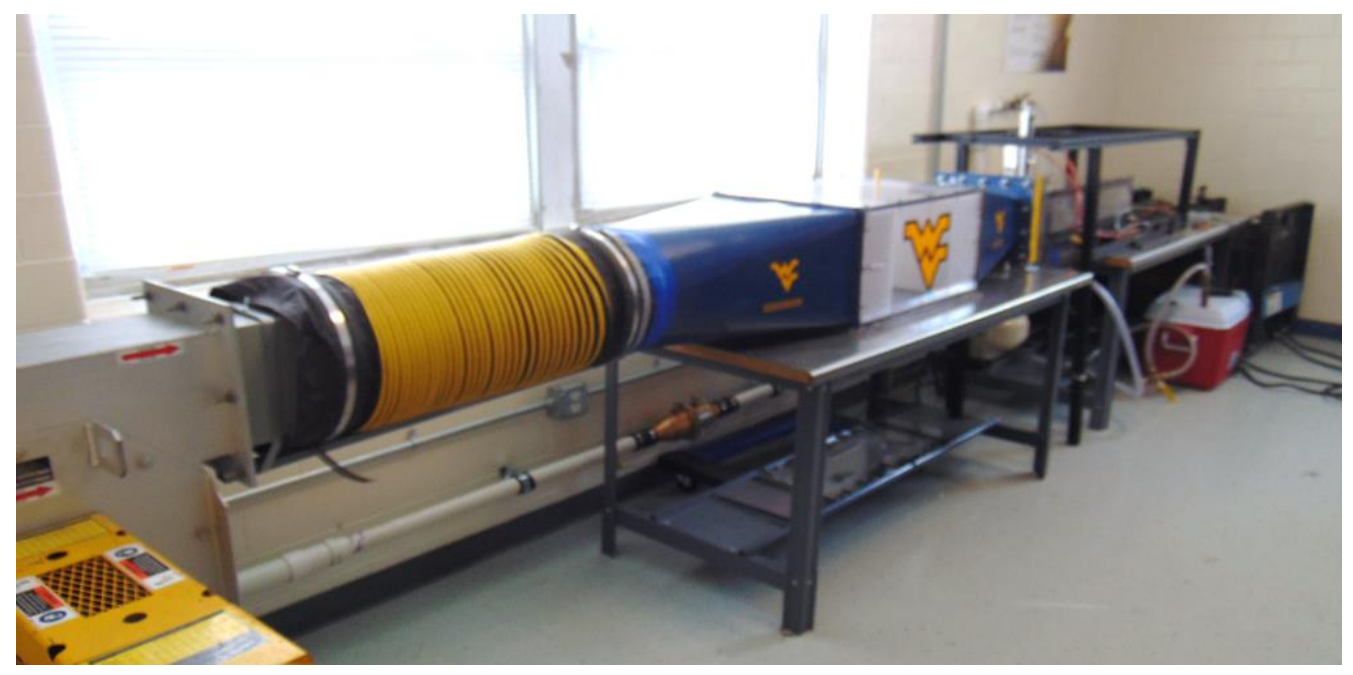

Figure 30 - Subsonic Low-Temperature Wind Tunnel 


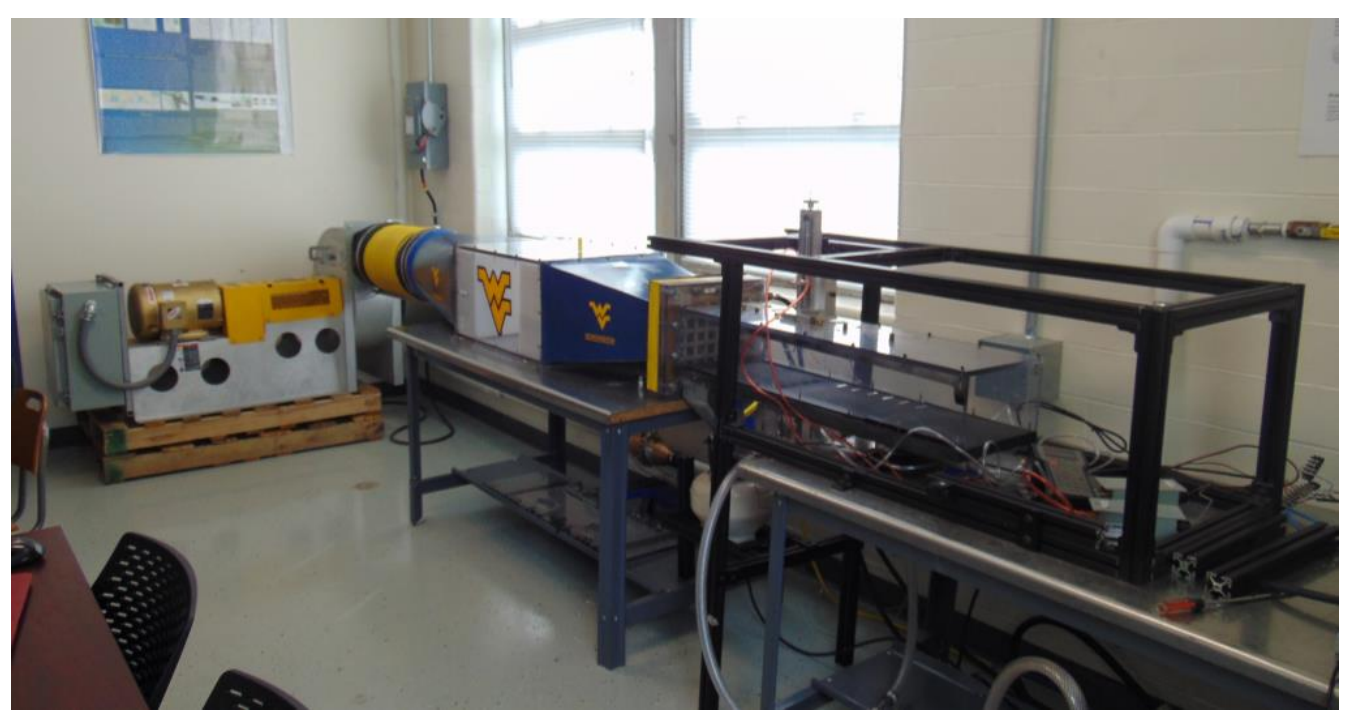

Figure 31 - WVU Subsonic Wind Tunnel at Test Section Exit

Downstream of the flow conditioner is a nozzle with a contraction ratio of 3.6:1 actively accelerating the flow depending on the fan revolutions per minute (RPM) setting. The flow is then further straightened as it passes through two 304 stainless steel wire mesh screens. These screens are connected in series to a Miller Deltaweld 451 MIG welder which provides the current necessary to increase the temperature of the screens thereby increasing the temperature of the freestream flow. This mesh screen is a $200 \times 200$ wire per square inch configuration with a 0.0016 -inch wire diameter and a 0.0034 inch on center wire spacing, thus yielding a resulting open area of $46 \%$ for each screen. Again these values were established and previously benchmarked by Hayes, et al. [23]. Hayes details that this setup yielded a nearly uniform temperature profile within the test section entrance. The mesh screen is connected via bus bar leads attached to the welder terminals and are capable of producing an increase of $10^{\circ}-20^{\circ} \mathrm{C}$ above ambient temperature conditions.

\subsubsection{TURBULENCE GRIDS AND TURBULENCE DECAY}

As mentioned, the flow before it reaches the turbulence grids approximately laminar. The turbulence grids allow a method to generate various levels of freestream turbulence intensities. This is accomplished by two lattice style passive grids which trip the flow to 
turbulent, based on data presented by Baines and Peterson [51]. As flow passes through the grid the bars act like bars in cross flow shedding specific sized eddies corresponding to the size of the grid bars.

Accurate prediction of grid performance in the tunnel is given by the theoretical decay of turbulence and theoretical turbulence length scale calculations.

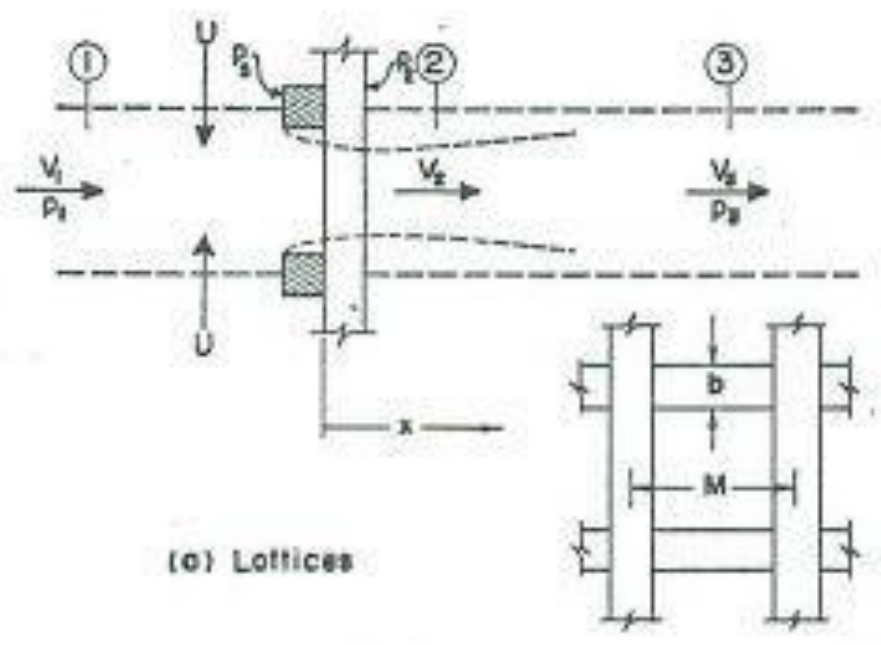

Figure 32 - Flow through Turbulence Grid [51]

Figure 32 above illustrates how flow through the grid would propagate to produce a series of jets through each opening between the bars that combine with the others resulting in quasicontrolled turbulent eddies developed from the shear over the bars. The decay of turbulence and the turbulent length scale, respectively shown below, can be predicted utilizing the following relations using the same terminology as in Chapter 2.

$$
\begin{aligned}
& T u_{x}=c\left(\frac{x}{b}\right)^{n} \\
& \Lambda_{x}=a b\left(\frac{x}{b}\right)^{m}
\end{aligned}
$$

Both the turbulence intensity decay and length scale decay are functions of the normalized stream wise distance, $\mathrm{x}$, as shown. The constants expressed in these equations 
are set as: $\mathrm{a}=1.12, \mathrm{c}=0.20, \mathrm{n}=0.53$, and $\mathrm{m}=-5 / 7$. The value of $\mathrm{b}$ then represents the bar diameter. This allows what can be considered the development of eventual isotropic turbulence as moving further away from the grid with decrement of turbulence intensity. Closer to the grids though the flow will still result in a laminar to transitional flow.

Based on previous work completed by Hayes, et al., documentation details various sized bar diameters and the resulting turbulence intensity vs a normalized streamwise length were found in the WVU facility before being decommissioned. These results shown in Figure 33initialized original turbulence bar fabrication and baseline turbulence values.
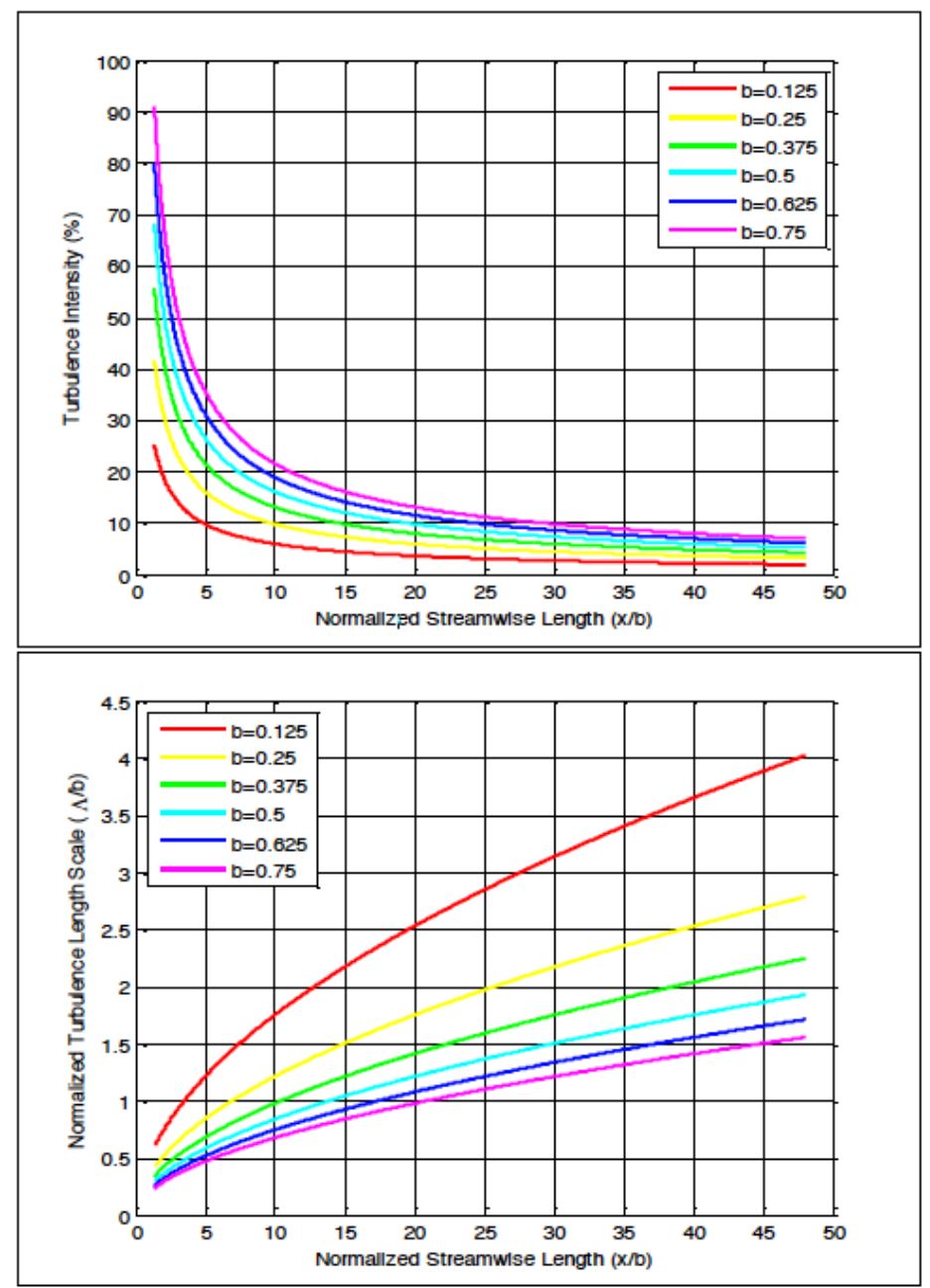

Figure 33 - Non-dimensional Turbulence Intensity and Length Scale for Multiple Bar Diameters [52] 
The bar grid diameter utilized in this investigation was set to $1 / 4 \mathrm{inch}$, and turbulence levels were evaluated with a unidirectional hotwire anemometer system. A $1 / 2$ inch bar section is also currently compatible with the test section mount as intended for flexibility in tunnel operations. It has been noted that the safe region for assuming isotropic turbulence is 5-10 bar diameters downstream of the grid section [51]. This location is where the facility allows various cooling hole geometries to be interchanged within the test section floor itself, for more rapid testing of various hole geometries. As previously noted the original facility construction noted data of the $1 / 2$ inch grid produced $11.7 \%$ freestream turbulence intensity at the cooling hole leading edge with a turbulence length scale of 1.4 nondimensionalized by the cooling hole diameter of 0.5 inch. The $1 / 4$ inch grid produced a freestream turbulence intensity of $7.5 \%$, with a turbulence length scale of 2.24 nondimensionalized by the cooling hole diameter. The turbulence intensity without turbulence grids was measured to be $1 \%$ and had a nondimensional length scale of 6.4 at the same respective location. Shown in Figure 34 below are the detailed geometric specifications of the grid sections.

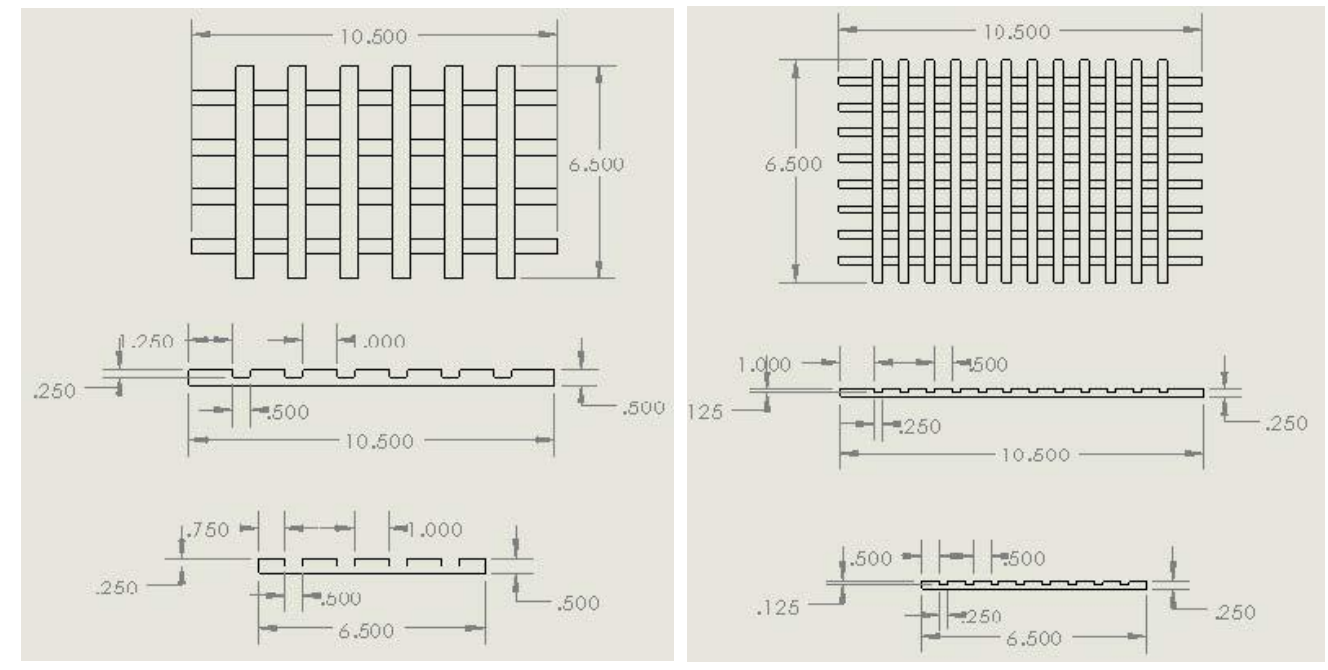

Figure $34-1 / 2 "$ and 1/4" Turbulence Grid Geometries

Figures 35 and 36 originally computed by Hayes et al. display freestream intensity and nondimensionally freestream turbulence length scale of trials completed experimentally at 
WVU and compared to Baines and Peterson's numerically predicted at nondimensional distances downstream of the grid bars for the $1 / 4$ " bar diameter [52].

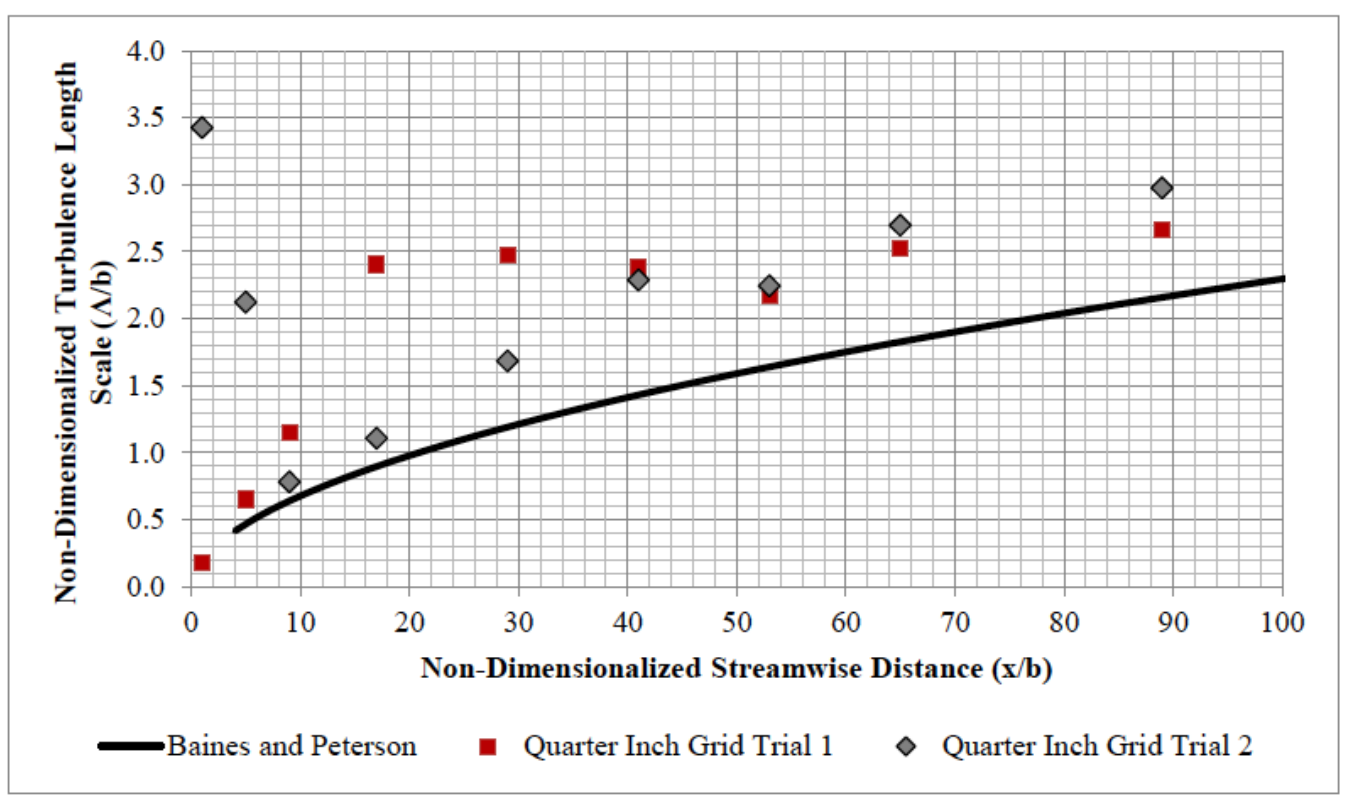

Figure 35 - 1/4" Grid Nondimensional Turbulence Length Scale [52]

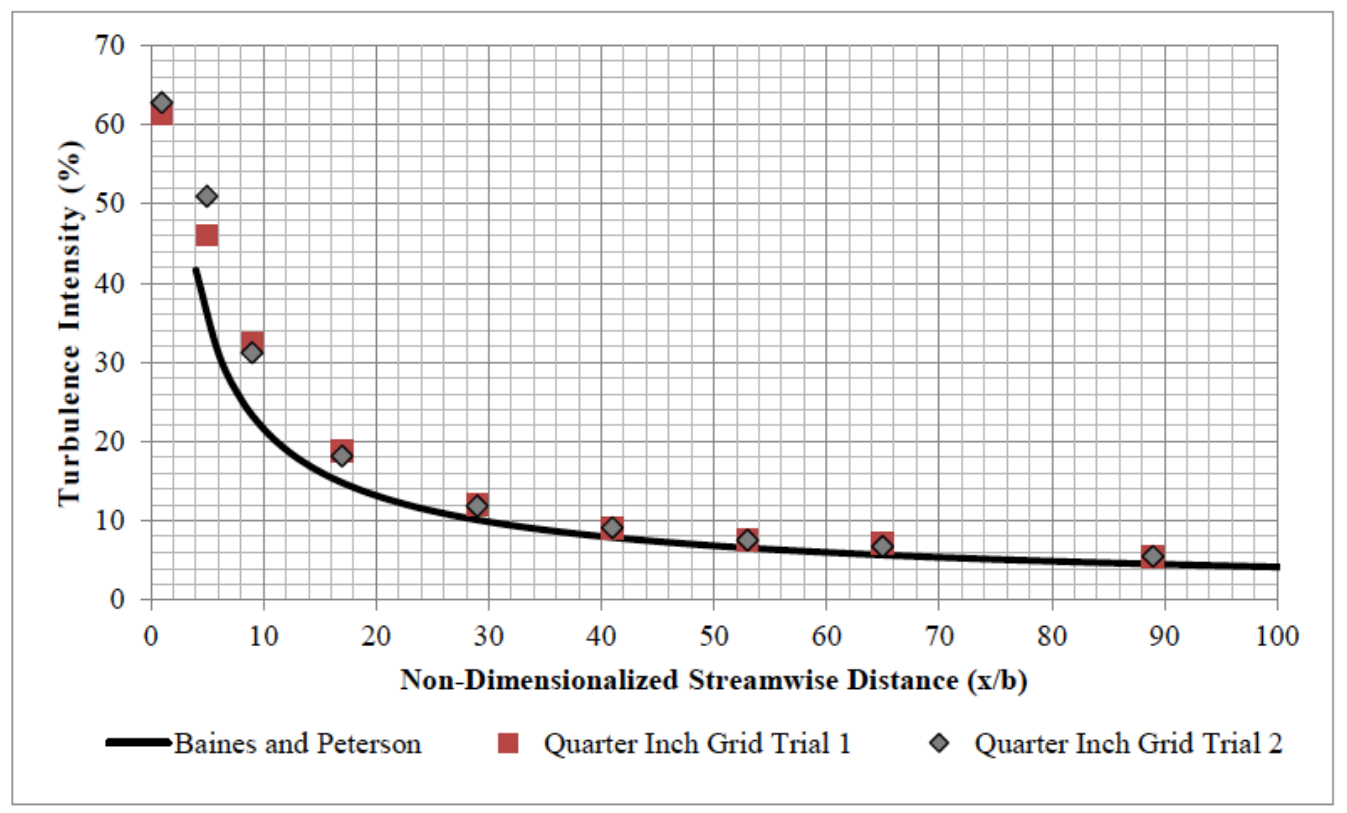

Figure 36 - 1/4" Grid Nondimensional Turbulence Intensity [52] 
The turbulence length scale is slightly underestimated by the Baines and Peterson approximation, whereas the turbulence decay values quite close to the given approach at the leading edge of the coolant holes. Hayes continued this evaluation for other bar diameters, however for the interest of this investigation, no further detail of proceeding results is included.

\subsection{TEST SECTION}

The test section of this wind tunnel is 6 inches by 10 inches in cross-section with a maximum flow velocity of $25 \mathrm{~m} / \mathrm{s}$. This section represents the region near the cooling holes on the suction and pressure sides of turbine airfoils. This section includes a section for various film cooling plates to be installed above an air supply plenum allowing multiple cooling hole geometries to be tested in future research. The plenum located under the cut-out section in the test section floor is also designed for future work when coolant air is required to simulate compressor bleed air for injection into cooling holes.

There are currently two different "lids" (top of test section) which may be used for different measurements that can be easily interchanged. The test section is comprised of $1 / 2$ inch Lexan side walls as well as two different $1 / 2$ inch Lexan lids. The first lid is entirely solid but is fitted with nine tapped holes for hotwire and pitot-static measurements at streamwise locations. The second lid is used for infrared thermography measurements possessing a milled slot replaced by a polyethylene window. The floor of the test section is comprised of 1inch thick ABS black plastic as Figure $37 \mathrm{CAD}$ drawing depicts below. 


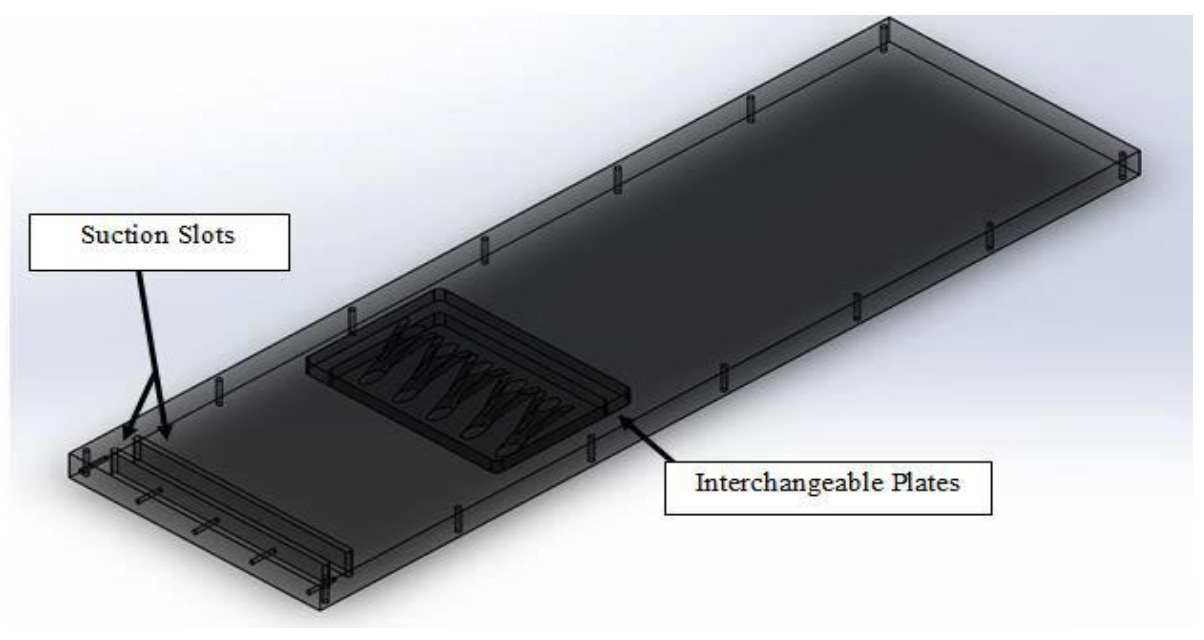

Figure 37 - Test Section Floor

The main feature of the test section base are the previously mentioned suction slots used for boundary layer thickness control. These suction slots run the width of the test section floor and allow a method of boundary layer thickness control. The WVU facility is able to match Reynolds numbers, but not realistically match the Mach number. For this reason, boundary layer suction is crucial to understand since the tunnel will produce a boundary layer not representative of conditions inside an actual gas turbine engine. The trailing edge of the last slot is 19 cooling hole diameters upstream of the plate insert where various hole geometries will be placed for future work. The slots are 0.5 -inch-wide in the streamwise direction spanning the width of the tunnel. The distance between the slots in the streamwise direction is approximately 0.75 inches. These slots are connected via an HVAC vent to a 4 inch PVC coupling to a RadonAway RP145 radon fan providing the necessary suction. Shown in Figure 38 (a) and (b) is the setup of the suction slots where Figure 38 (a) specifically shows the suction slots in the test section floor, and Figure 38 (b) depicts the Radon fan and HVAC/ PVC hookup with the fan disconnected. 


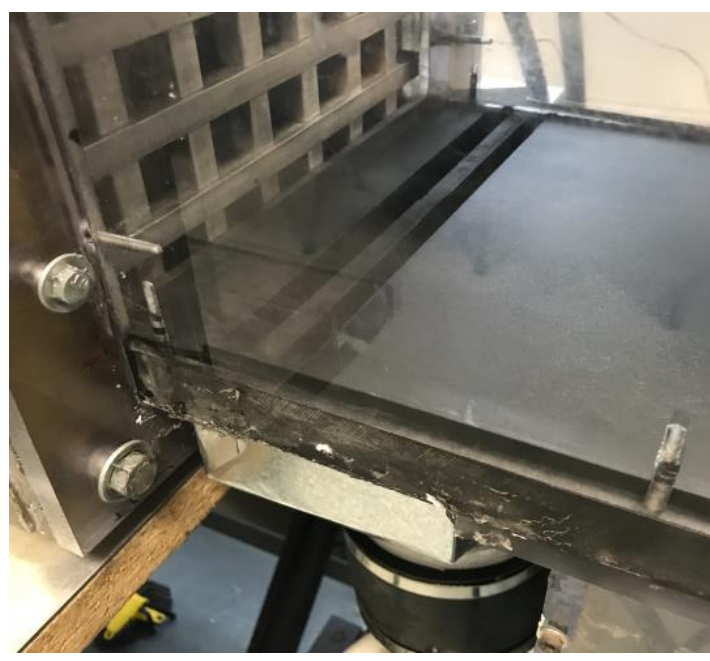

(a)

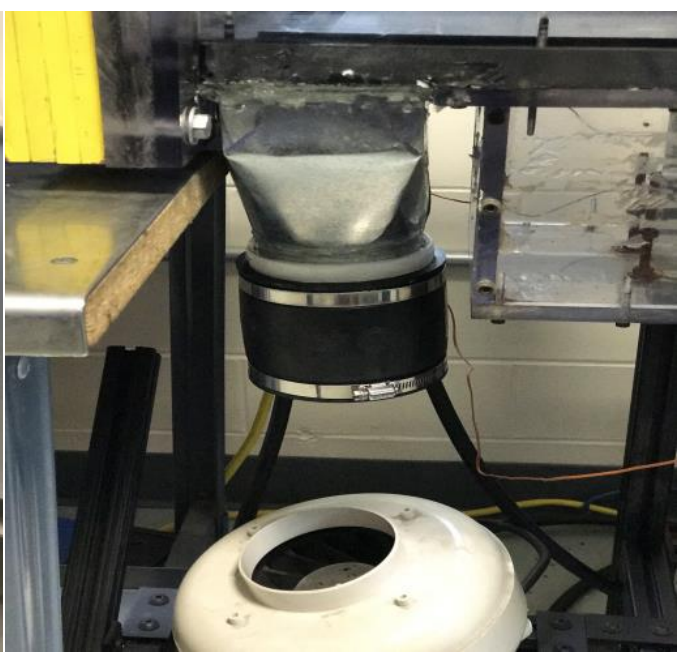

(b)

Figure 38 - Suction Slot Setup

Several refined increment pitot-static probe traverses were vertically completed at varying locations along the length of the tunnel to determine the freestream velocity profile, and along with knowing the test section dimensions, the mass flow rate as well. Figure 39 shows the setup required for completing the traverse with a hotwire anemometer and a pitotstatic probe. The traverse is a Velmex unislide A2509P40 fine increment traverse system with measurements given by combining the readings from the linear scale and engraved knob. The resolution of the knob measures position from 0.0001 inch. 1 complete revolution of the knob equates to $1 / 40$ linearly measured inch.

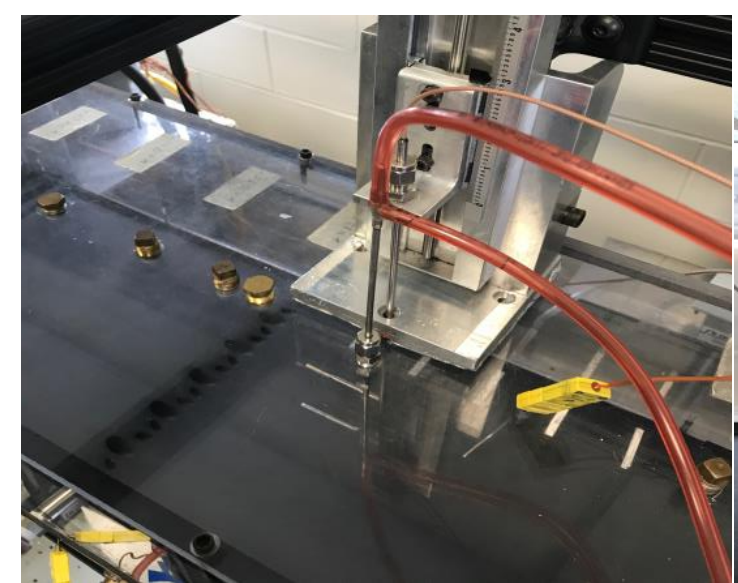

(a)

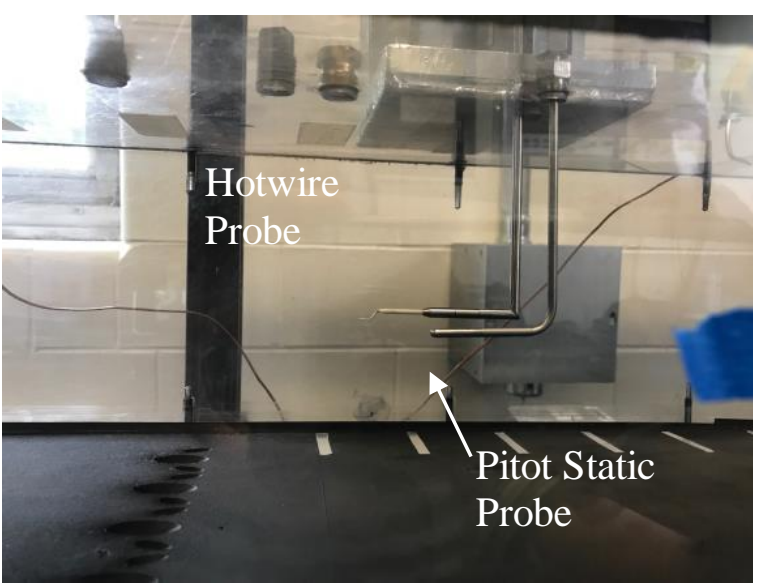

(b)

Figure 39 - Hotwire Setup 
While this experimental setup previously was operational at West Virginia University, the components had to be disassembled, transported to a new facility, and then reassembled in a new location. This was a long process involving running new piping lines, repairing and replacing damaged equipment, and reactivating the data acquisition system. Additionally, new data acquisition code was written in order to compensate for the modifications in changed data ports. Continuous improvement is ongoing at the conclusion of this investigation to upgrade the boundary layer suction fan, implement an electronic controller for the suction fan to vary the amount of suction, and implement an electronic connection to interface control of the blower into Labview $®$ code directly. 


\section{CHAPTER 4: DATA COLLECTION \& CALIBRATION}

\subsection{DATA AQUISITION COMPONENTS}

All collected data including hotwire, pressure, and temperature data was recorded through a series of National Instruments LabVIEW ${ }^{\circledR}$ codes. In Figure 40 the four module Ethernet connected, compact $9184 \mathrm{DAQ}$ is shown. The pressure and temperature data are collected simultaneously during the transient test. The module furthest to the left is the four channel NI 9215 BNC connection module used to collect hotwire data. The middle two modules are NI 9211 thermocouple modules each with four channels. The furthest right module is a four channel NI 9215 module with a screw terminal for connection to the pressure sensors.

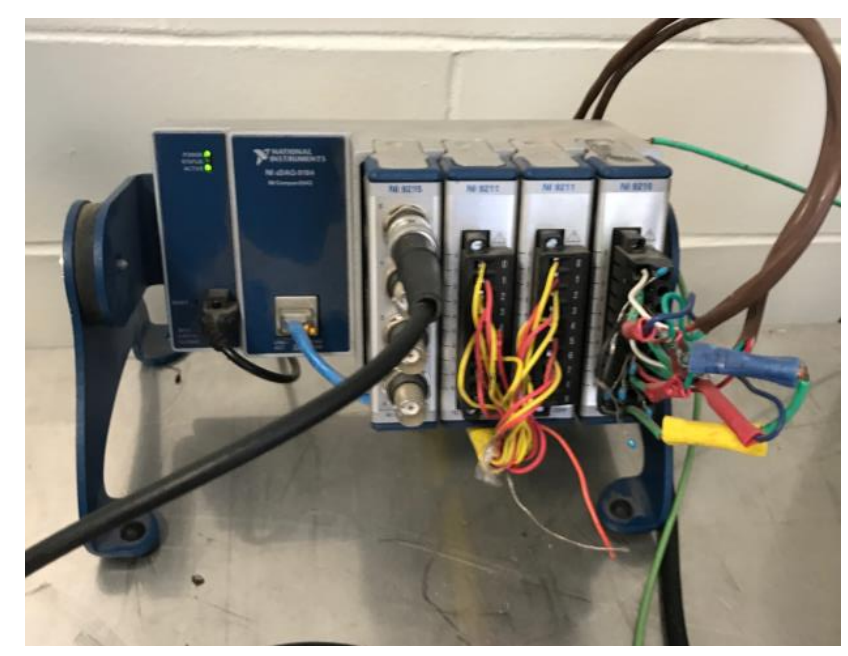

Figure 40 - Data Acquisition System

\subsubsection{OMEGA THERMOCOUPLES}

Setup of the recommissioned tunnel includes thermocouples mounted in various locations in the test section surface and air plenum sections. The thermocouples used in this investigation were Omega Engineering KMQSS-020E-6. These K type thermocouples are comprised of dissimilar metals, of CHROMEGA® and ALOMEGA®, which when experiencing a temperature difference produce a voltage correlating to the temperature. This 
type is commonly used in lab-based settings with a variety of applications. This facility employed the thermocouples in a manner that exposed the tips allowing for a minute connection of $<0.020$ " between both metals. This allows for a faster response time with a time constant of less than half a second to changes in temperature than a covered tip.

\subsubsection{OMEGA PRESSURE TRANSDUCERS}

The differential pressure transducer used for this experiment was an Omega PX27705D5V transducer for pressure measurements by the pitot-static probe for velocity and dynamic pressure for the boundary layer probe as the low-pressure side remained open at atmospheric pressure. The accuracy of this transducer was $\pm 1 \%$ of the selected field. This pressure transducer has an accuracy of $\pm 0.25 \%$ of the "frequency response best straight line". Connection for this data process was through an NI 9215 four-channel analog input card. Power was supplied to the transducer through a 0-30 Volt (V), 0-3-amp (A) variable benchtop power supply; where the set voltage was established at $20 \mathrm{~V}$ to maintain necessary current.

The pressure transducer was calibrated using a linear relationship between the output voltage of the sensor and the corresponding pressure. This system channel calibration is conducted using two known points, one at zero and the other at an arbitrary value found using a digital pressure manometer. Solving this pair of linear equations yields the unknown slope and intercept value for the linear relationship. This calibration equation was then inserted into the LabVIEW ® code.

\subsubsection{DANTEC CTA HOTWIRE}

Turbulence data was collected using a Dantec Dynamics $5 \mu \mathrm{m}$ one-dimensional gold plated boundary layer hotwire anemometer. The probe and Wheatstone bridge setup, rated for a maximum sampling rate of $400 \mathrm{kHz}$, was connected to the National Instruments 9215 BNC 4 channel analog input module with a maximum sampling rate of $100 \mathrm{kHz}$. In order to satisfy the Nyquist frequency (defined as half the maximum sampling rate to avoid 
aliasing), data was collected at $50 \mathrm{kHz}$. This sampling rate was chosen based on previous tests conducted in the facility prior to recommissioning, and was previously established on the theoretical eddy integral length scale concluded from turbulence grid bar diameters and the mean flow velocity. The advantage of the boundary layer probe is that it is bent slightly to allow access to the boundary layer without creating a pressure front greatly disrupting the flow closer to the test section surface. The exact model boundary layer probe used was a Dantec Dynamics 55P15 probe, shown in Figure 41 below.
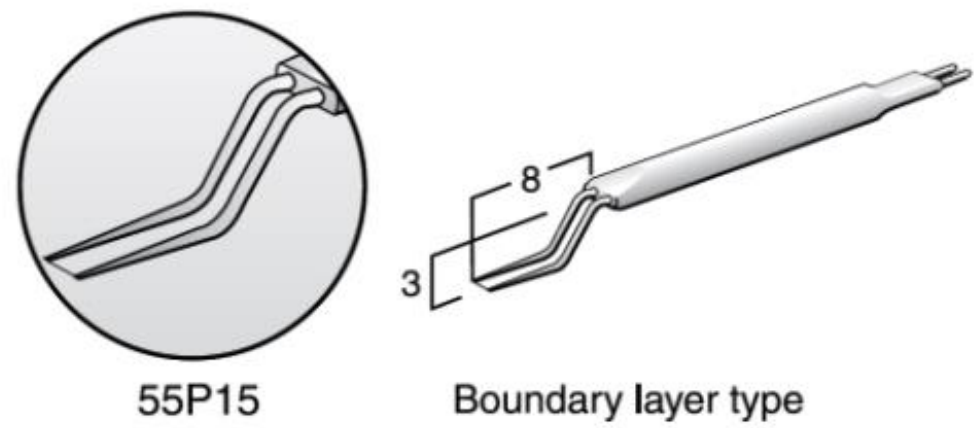

Figure 41 - Boundary Layer Probe [53]

Calibration of the boundary layer hotwire was conducted by comparing data with the pitot-static probe. This was conducted in a large range of data and applied to King's Law equation as shown [53]:

$$
V^{2}=m U^{n}+b
$$

The freestream velocity, $\mathrm{U}$, is raised to the power $\mathrm{n}$ representing the Kings Law exponent, $\mathrm{n}$, typically between 0.45 and 0.5 ; chosen here as 0.45 . This relates to the squared voltage, $\mathrm{V}$, by constants $\mathrm{m}=.139$ and $\mathrm{b}=-.230$, the slope of the calibration curve and the calibration constant respectively. The calibration curve for this experiment is shown in Figure 42 below. The velocity range that is covered by this calibration goes from $0 \mathrm{~m} / \mathrm{s}$ to $\max$ rotational setting of the motor/fan which is roughly $25 \mathrm{~m} / \mathrm{s}$. 


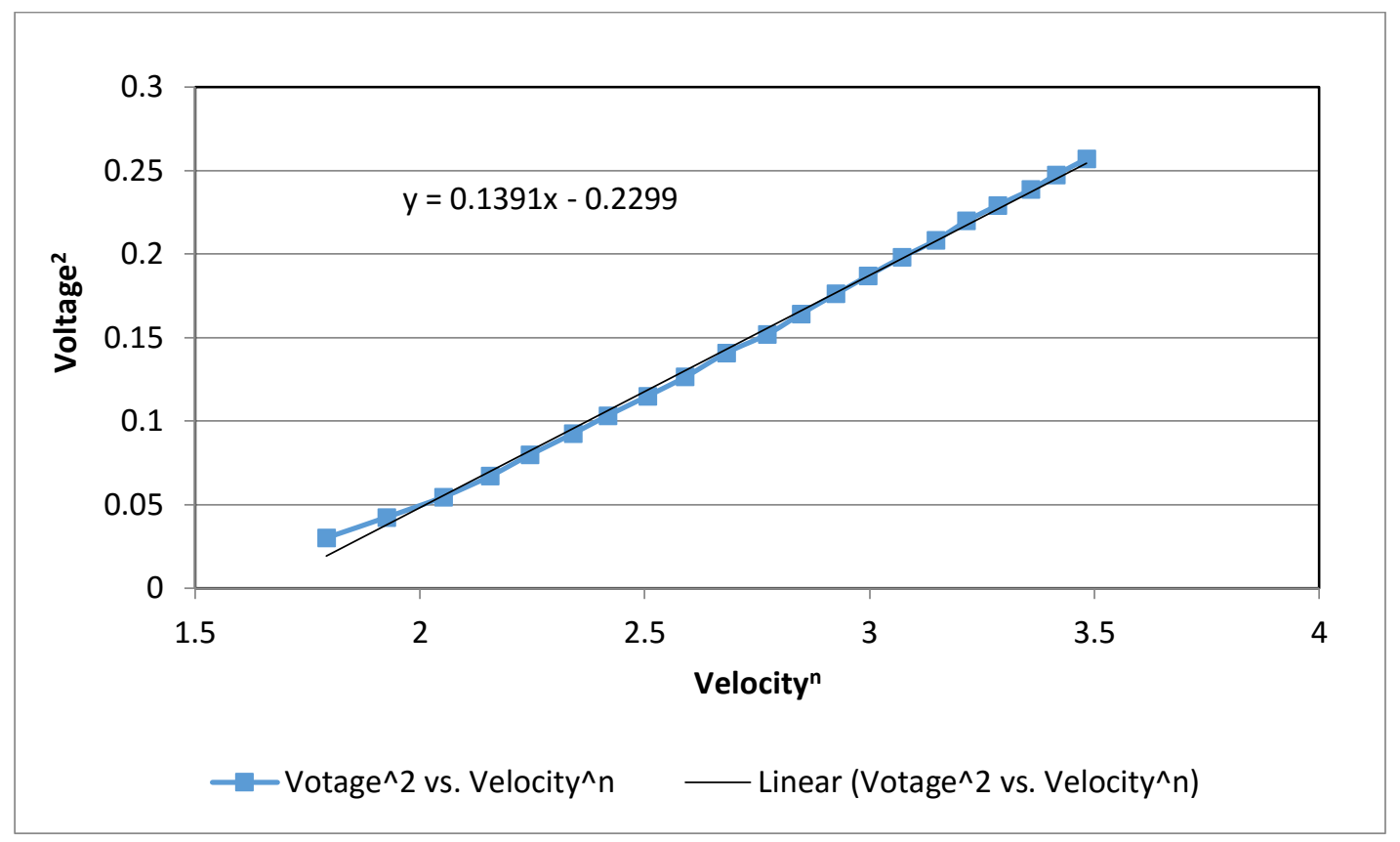

Figure 42 - CTA Calibration Curve

The freestream velocity is found through the use of the pitot-static probe by using Bernoulli's equation to relate the dynamic pressure and the static pressure:

$$
P_{\text {static }}+\frac{\rho U^{2}}{2}=P_{\text {total }}
$$

leading to the useful equation solving for $\mathrm{U}$ :

$$
U=\sqrt{2 * \frac{\Delta P}{\rho}} \quad \text { where } \Delta P=P_{\text {total }}-P_{\text {static }}
$$

All of the calibration data was obtained at the centerline position inside the wind tunnel test section at various blower rotational speeds. Upon completing the calibration, the hotwire CTA is used to measure both the average velocity and the high-frequency velocity fluctuations of the freestream flow, then from these values the turbulence intensity and integral eddy scale is determined. 


\subsection{UNCERTAINTY}

The uncertainty of data obtained with the anemometer is a combination of the statistical analysis of the velocity arrays collected, and that of the individually acquired voltages then converted into velocity values. The uncertainty of each velocity sample can be found based on knowledge of instrumentation specifications, the equipment used for calibration, and conditions experienced during the experiment. The uncertainty of each velocity sample can be found based on knowledge of instrumentation specifications, the equipment used for calibration, and conditions experienced during the experiment. The anemometer itself has low drift and noise, with high repeatability, ideal for experiments such as the present work.

As considered a general norm with engineering applications, a Gaussian error distribution combining the uncertainties obtained from voltages and the statistical analysis is used [53]. The total relative uncertainty is defined as:

$$
U=2 \sqrt{\sum u(y)^{2}} \text { where } u(y)=\frac{1}{y_{i}} S\left(\frac{\Delta x_{i}}{k_{i}}\right)
$$

where $\mathrm{u}(\mathrm{y})$ is the relative standard uncertainty, $\mathrm{S}$ is the sensitivity factor defined for this experiment as $0.125^{-1}$, and ki equals 2 representing the coverage factor related to the Gaussian input variance.

The calibration uncertainty, $U_{c a l}$, is defined as the percent of the standard deviation of the calibration velocity:

$$
U\left(U_{\text {cal }}\right)=\frac{1}{100} \operatorname{STDV}\left(U_{\text {calibrator }}(\%)\right)
$$

A linearization uncertainty, $U_{\text {linear }}$, associated with the curve fitting errors in the calibration points expressed as the standard deviation in percent:

$$
U\left(U_{\text {linear }}\right)=\frac{1}{100} S T D V\left(\Delta U_{\text {lin }_{\text {calibration }}}(\%)\right)
$$


The data acquisition board has an uncertainty, $U_{\text {res }}$, due to manufactured resolution expressed as a function of the sensitivity factor of the inverse calibration curve, $\frac{\partial U}{\partial E}$, the A/D board input range, $\mathrm{E}_{\mathrm{AD}}$, and the resolution in bits, $\mathrm{n}$.

$$
U\left(U_{\text {res }}\right)=\frac{1}{\sqrt{3}} * \frac{1}{U} * \frac{E_{A D}}{2^{n}} * \frac{\partial U}{\partial E}
$$

Uncertainty contributed by probe positioning, temperature variations, ambient pressure variations, and gas composition caused by changes in humidity can be neglected for this study as the facility is climate controlled to mitigate variations in these parameters. The described values are detailed in Table 5 below, along with a total uncertainty, $U_{t o t}$.

\section{Table 5 - Uncertainty Variables}

\begin{tabular}{|c|c|}
\hline Parameter & Value \\
\hline $\boldsymbol{E}_{\boldsymbol{A D}}[$ volts] & 21 \\
\hline $\mathrm{N}[\mathrm{bits}]$ & 32 \\
\hline$\frac{\partial U}{\partial \boldsymbol{E}}[\mathrm{m} / \mathrm{s} /$ volts] & 7.19 \\
\hline$\left(\boldsymbol{U}_{\text {cal }}\right)[\%]$ & \pm 2 \\
\hline $\boldsymbol{U}_{\text {linear }}[\%]$ & 0.5 \\
\hline $\boldsymbol{U}_{\text {total }}[\%]$ & $\approx 4$ \\
\hline
\end{tabular}

This value, $\mathrm{U}_{t o t}$, is arrived at via quadrature, and does not include errors generated by user measurement taking data called systematic error, nor does it account for randomization errors. However, the systematic error is accounted for upon consideration that the traverse increment motion through each step change was performed by the same individual with the same reading methodology. This allows for the data to at least be accurate if not necessarily precise. Additionally, the random error associated with measuring instrumentation or environmental conditions is not deemed significant as Chauvenet's criterion is applied. This 
criterion provides a procedure allowing the removal of any inconsistencies in general overall data trends as per the criterion algorithm further detailed below.

During data collection, there were spikes in data that appeared systematic appearing regularly in intervals of every 11 testing samples. This frequency is most likely attributed to the vibration of the probe in the tunnel as the probe support drag is orders of magnitude greater than the probe itself. In order to process these "outliers" out from the majority of the data, a code was written utilizing Chauvenet's criterion. This statistical methodology allows "negation" of data points which lay outside a probability band, centered on the mean of a normal distribution. In other words, should data lay outside a bandwidth multiplier of standard deviation normally a multiplier of 2 , it can be regarded as a "false" data point leaving $95 \%$ of the data, as is customary, within two standard deviations of the mean. Since procedural data collection occurred in the same fine increment step sizes of each case, it was determined that in order to keep data array lengths consistent among experiments a linear interpolation was conducted to substitute in these values deemed outside of Chauvenet's criterion. 


\section{CHAPTER 5: EXPERIMENTAL RESULTS}

\subsection{EXPERIMENT DESCRIPTION \& SETUP}

The aim of this investigation is to document boundary layer properties within four scenarios. The first scenario is the case of little to no turbulence (dubbed laminar trials) within the boundary layer and the suction fan off. This is followed by the scenario of maintaining the existing flow but introducing the boundary layer suction. Scenarios three and four follow the same procedure of suction on and off respectively, however introduce a turbulence generator within the boundary layer on the test section floor. The turbulence generator was a piece of tape inserted at the trailing edge of the second suction hole and raised 0.3150 inches from the test section floor into the flow. The turbulence generator was located therefore approximately 19 cooling hole diameters ( 9.5 inches) upstream of the cooling hole test plate. This location from another reference is approximately 4.75 inches upstream from the hotwire probe. A disturbance such as this within the boundary layer should generate wakes which will turn into turbulence downstream near the probe location. All four scenarios experienced similar freestream turbulence so as to not vary freestream effects on the boundary layer. The freestream turbulence was generated by passive turbulence bars at the entrance in the tunnel as detailed in Chapter 3: Experimental Setup.

The testing commenced with calibrating the boundary layer hotwire CTA system and pressure transducer for use with a pitot-static probe. Detail of calibration for both the pitotstatic probe pressure transducer and the hotwire CTA module are described in Chapter 4 at the centerline tunnel location. Following calibration MATLAB ${ }^{\circledR}$ and Labview ${ }^{\circledR}$ codes were written for data acquisition and post processing.

The flow measurements were started along the bottom surface of the test section and traversed up as further detailed in the proceeding section. Each test was conducted at a blower setting of $2400 \mathrm{RPM}$, producing test section average velocities of roughly $10 \mathrm{~m} / \mathrm{s}$. Shown in Figure 43 below is the test section near the suction slots. 


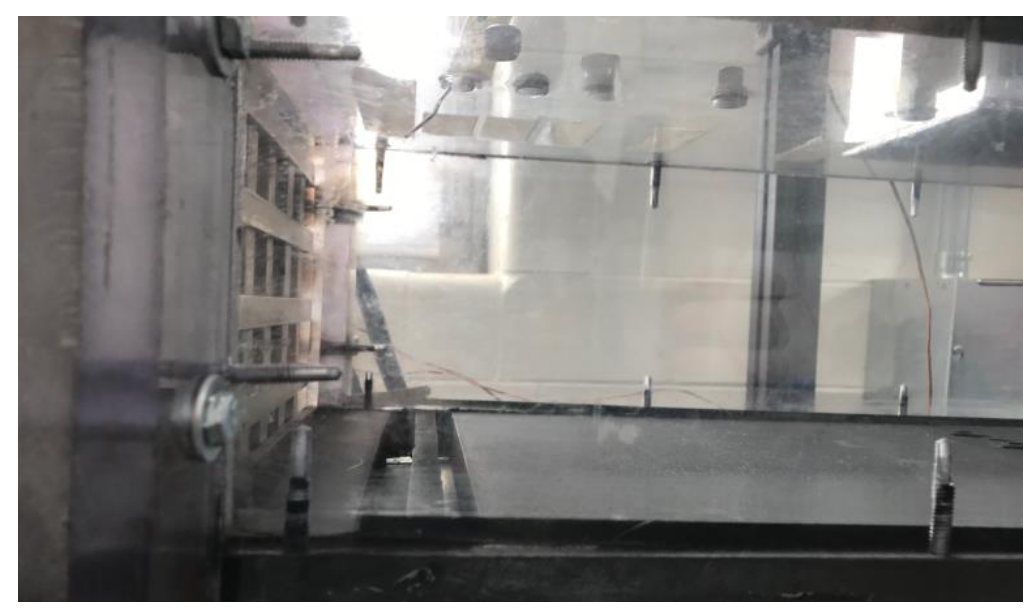

Figure 43 - Suction Slots and Test Region without Probes

\subsubsection{TESTING PROCEDURE}

The position of the hotwire was horizontally centered in the test section to prevent influence from the vertical walls on either side. The flow measurements near the test section floor were taken 9.625 inches downstream of the turbulence grid location. The first suction slot is $1 \mathrm{inch}$ from the test section entrance with a second suction slot leading edge 2.25 inches from the test section entrance. Using a broken probe to set the starting position just above the test section surface, the actual probe was traversed up 0.0075 inch for the first 10 data points, increasing to increment step sizes of $0.0375 \mathrm{inch}$ until freestream conditions were met signifying the edge of the boundary layer. The distance of the total traverse was from the location just above the test section floor (the datum point) to $0.15 \mathrm{inch}$ past the freestream conditions. Each data point as presented was collected by averaging transient data recorded on a dwell time of 10 seconds by the DAQ system.

\subsection{RESULTS AND DISCUSSION}

\subsubsection{LAMINAR FLOW}

The boundary layer thicknesses for the non-tripped laminar scenarios were found to be 1.045 inches when the suction motor was turned off, and 0.74 inch when the suction fan was 
turned on. The non-dimensional value of the boundary layer height over the cooling hole diameter, $\delta / D$, for the no suction and suction on cases were 2.09 inches and 1.59 inches respectfully; where the cooling hole diameter, $\mathrm{D}$, is again noted as $0.5 \mathrm{inch}$. While achieving a reduction of this parameter value by implementing suction, these results are nowhere near the targeted values of Whitlow, as detailed in the latter portion of this section. The suction fan provides a change of nearly a quarter of an inch in boundary layer thickness. In order to find all the listed parameters in Table 6, a MATLAB $®$ code was written using the equations listed in the previous sections. Data presented in Table 6 is given to three significant digits for results such as the momentum thickness, displacement thickness, and shape factor. The Reynolds number for these scenarios based on the entrance length to the hotwire is in the region of $1 \times 10^{5}$.

Table 6 - Non-Tripped Laminar Results

\begin{tabular}{|c|c|c|}
\hline Property & No Suction & Suction \\
\hline $\boldsymbol{\delta}[\mathbf{i n}]$ & 1.05 & 0.800 \\
\hline $\boldsymbol{\delta} / \boldsymbol{D}[\mathbf{i n}]$ & 2.09 & 1.59 \\
\hline $\boldsymbol{\delta}^{*}[\mathbf{i n}]$ & .117 & .0730 \\
\hline $\boldsymbol{\theta}[\mathbf{i n}]$ & .0890 & .0570 \\
\hline $\boldsymbol{H}$ & 1.32 & 1.27 \\
\hline $\begin{array}{c}\text { Tu just inside boundary layer } \\
{[\%]}\end{array}$ & $21.4 \%$ & $19.8 \%$ \\
\hline $\boldsymbol{\Lambda}$ just inside boundary layer & & 3.27 \\
\hline [in] & .669 & \\
\hline
\end{tabular}

The length scale, $\Lambda$, as referred to in Table 6 as just inside the boundary layer, specifically details the length scale within two increment step changes inside where the apparent boundary layer location was. The turbulence intensities as listed in Table 6 are within two 
increment step changes inside the assumed boundary layer location as well. By the apparent similarity between all scenario turbulence intensities, it can be assessed that the boundary layer may not have been in the location as prescribed by the given definition of $99 \%$ freestream conditions. The length scale for the suction scenario of 3.27 inches, is definitely unreasonable and isn't possible for the experiment conducted. Data was doubled checked, but needs recollected in order for validation. This erroneous value could be attributed to error in data collection, but most also likely an error in data post-processing. However, further speculation as to this large value will be paused until presentation of the spectral analysis where additional data irregularities exist for this particular case.

Below in Figures 44 and 45 are the velocity profiles that resulted from the hot wire traverses for suction on and off respectively for a non-tripped laminar boundary layer. Typical of a laminar profile, Figure 44 has the attributes yielding that the boundary layer just upstream of the coolant hole section is laminar in general shape with minor fluctuations.

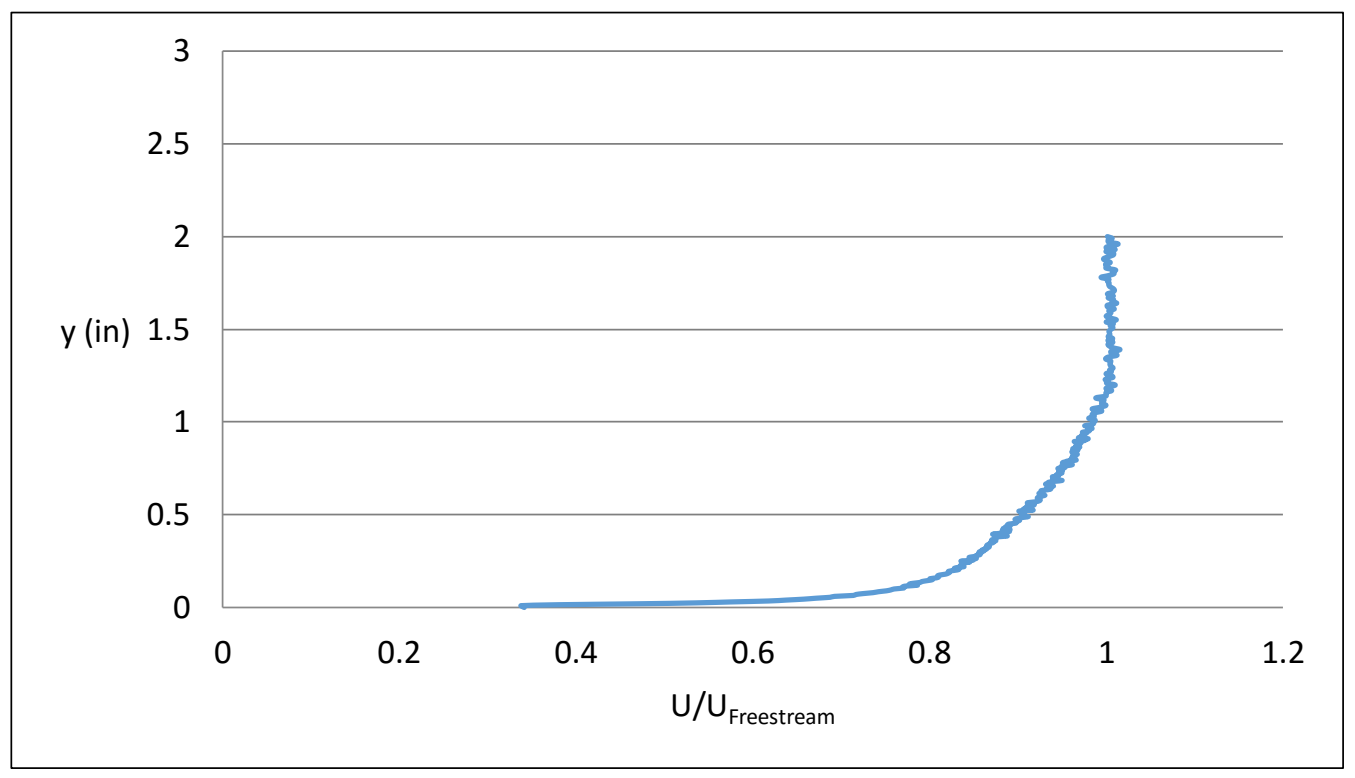

Figure 44 - Traverse Height Velocity Profile Turbulence OFF and Suction OFF 


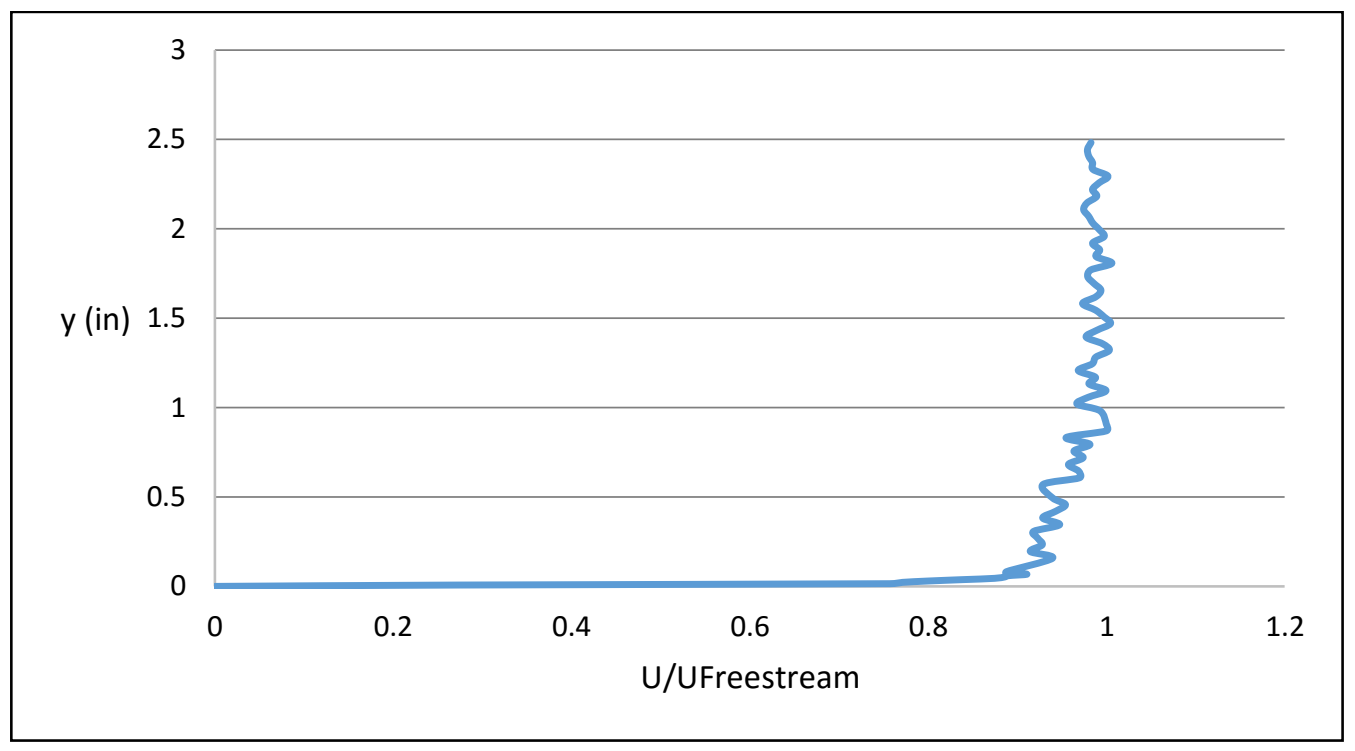

Figure 45 - Traverse Height Velocity Profile Turbulence OFF and Suction ON

Due to strictly the holding the definition of the boundary layer as $99 \%$ freestream conditions, this yields values of the boundary layer height slightly higher than previously benchmarked by Whitlow [45] at WVU prior to tunnel decommissioning. Using similar techniques as this investigation, Whitlow found the tunnel boundary layer without suction to be 1.5 inches, while turning on the suction reduced the boundary layer to approximately 0.25 inch [45]. Shown in Figure 46 below are Whitlow's comparison the boundary layer before and after introducing suction. While this investigation did not match exactly previous benchmarks most likely attributed to differences in testing and setup, overall data trends and reduction in boundary layer parameters were achieved. 


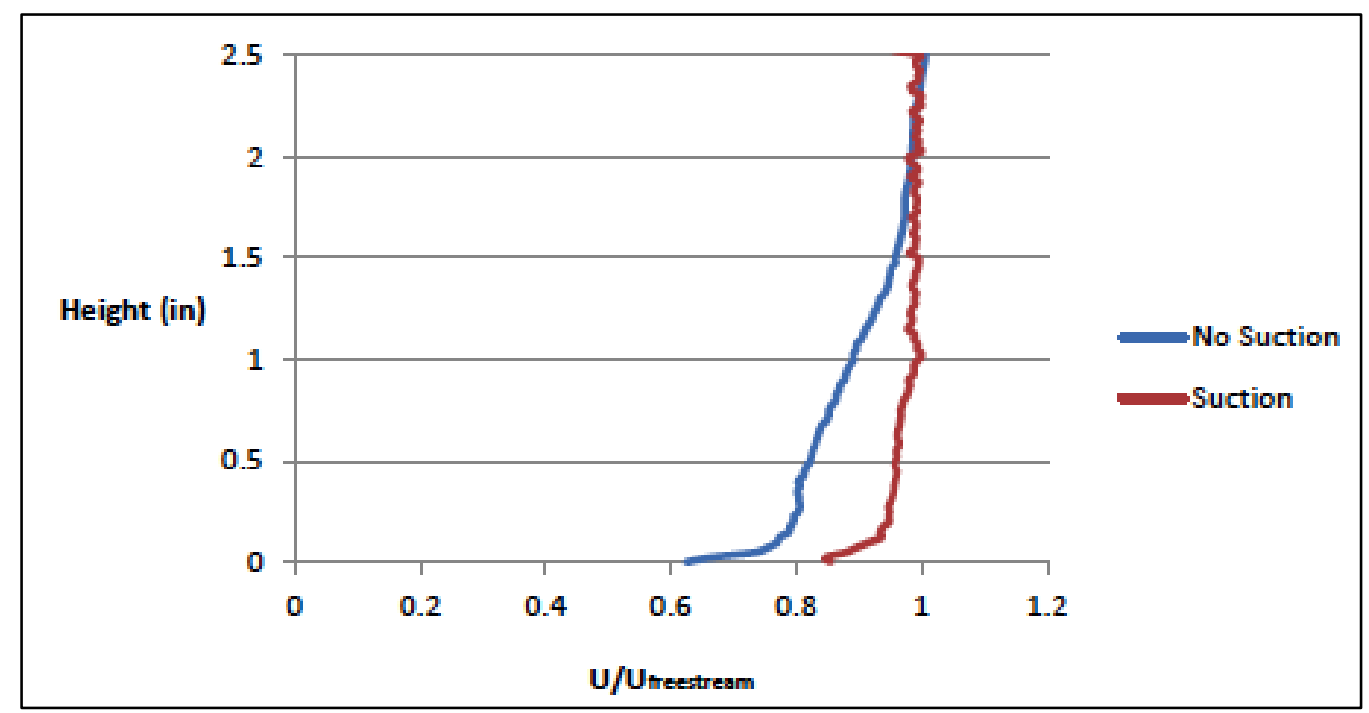

Figure 46 - Whitlow WVU Boundary Layer Velocity Profile

Comparison [45]

Next, to begin the turbulence analysis an autocorrelation of the velocity signals is completed. This information details whether or not the flow inside the boundary layer is infact turbulent. In the case of the tripped flow, turbulent conditions are expected. Therefore, for additional comparison, the autocorrelation is completed at three primary locations: inside the boundary layer at the set zero point, the believed boundary layer location based on the 99\% definition proposed, and 0.15 inch outside the boundary layer. This three step comparison is completed for each of the four testing scenarios in the aims of comparing turbulence data within, at, and outside the boundary layer for flow regime classification. It is expected that since the freestream flow is turbulent, the turbulent analysis should undoubtedly yield results that are characteristic of a turbulent flow.

In order to compute the length scale, the autocorrelation is needed as just detailed. Utilizing the approach used by Nix [54], another MATLAB $®$ code was written and adapted for this analysis. Figures 47-49 detail the autocorrelations for the test (a) of suction OFF and (b) suction $\mathrm{ON}$, at the hotwire location near the test section floor, at the believed boundary layer height, and 0.15 inch outside the boundary layer respectively. Note again the location outside the boundary layer shouldn't be significant since it is expected that the flow 
everywhere in this regime should be turbulent. Utilizing the autocorrelation, the integral time scales are found from seeking the first zero crossing. Zoomed in magnifiers on Figures 4749 show the respective zero crossing for each case.

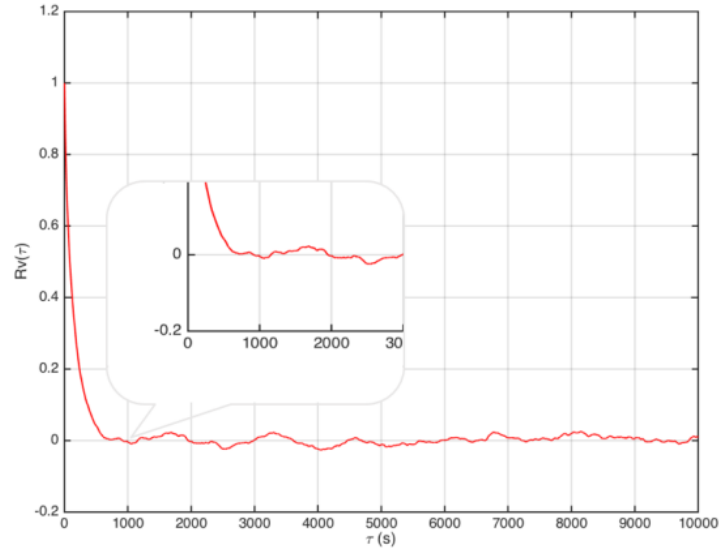

(a)

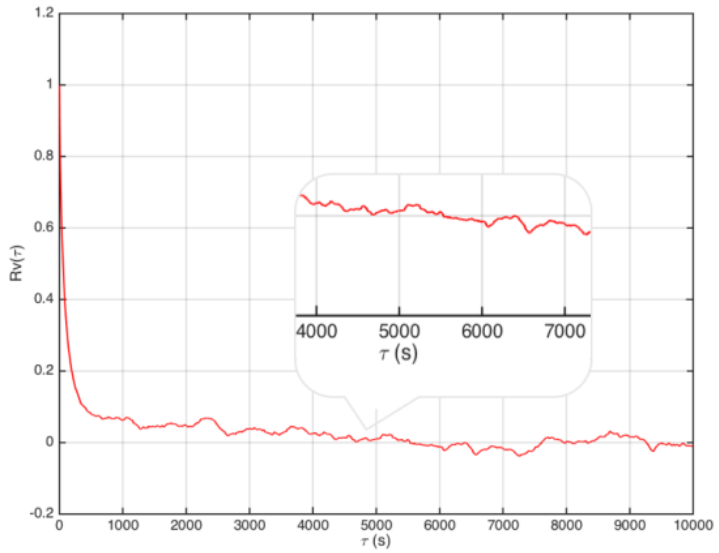

(b)

Figure 47 - Inside the Boundary Layer Autocorrelation Plot No Turbulence Trip for Suction. OFF (a) and Suction ON (b)

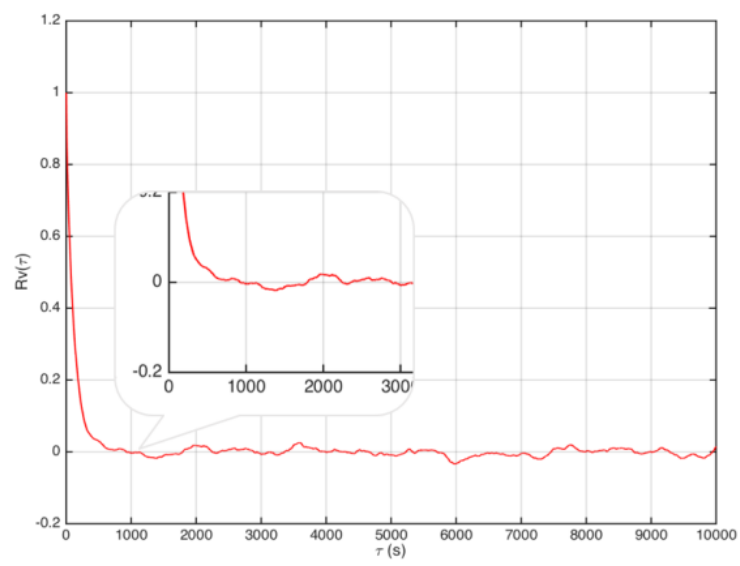

(a)

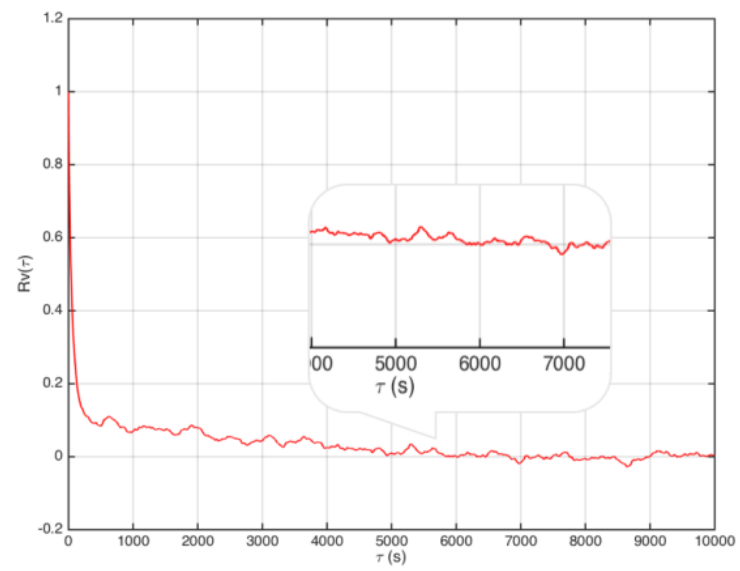

(b)

Figure 48 - At the Boundary Layer Autocorrelation Plot No Turbulence Trip.

Suction OFF (a) and Suction ON (b) 


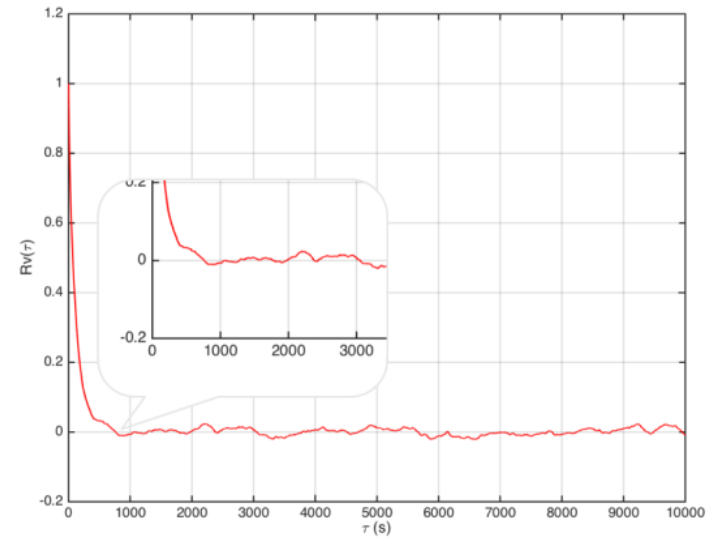

(a)

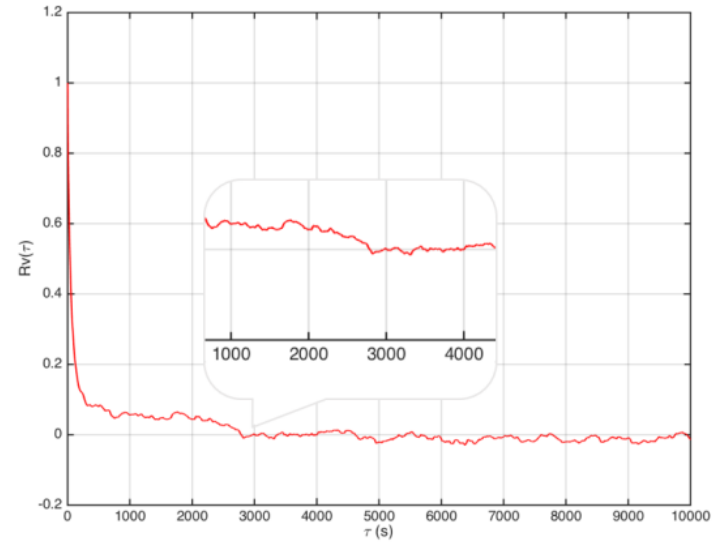

(b)

Figure 49 - Outside the Boundary Layer Autocorrelation Plot No Turbulence Trip.

Suction OFF (a) and Suction ON (b)

The results shown in Figure 47-49 start to help understand the presented turbulence characteristics. The first zero crossing is really the limiting point of interest; as noise is generally thereafter the only concern in the data. The noise here would be contributed to an effect of dominant peaks in the frequency domain appearing as small amplitude, high frequency vibrations caused from the hot-wire probe support. Further inspection reveals only small amplitude peaks exist after the zero crossing. Therefore, integrating the autocorrelation beyond the zero crossing may inflate the time scale value erroneously. Inspection of Figures 47-49 shows that when the suction is turned on (i.e. Figures 47(b) - 49(b)) the zero crossing is a larger value of $\tau$ as contrasted with the suction off scenarios of Figures 47(a) - 49(a).

Figures 50-52 represent the power spectral density of the velocity signals, or how much kinetic energy is present on average at a particular time scale. Averaging the frequency of the power spectral density is plotted in Figures 53-55 detailing the generated eddies' average kinetic energy at the respective locations. The trailing edge of Figures 53-55 shows little peaks consistently and periodically appearing throughout the data, attributed to the dips in voltage readings throughout testing. Initial findings yield that while the boundary layer thickness is decreased, overall turbulence isn't greatly reduced. However, since these figures 
represent the two scenarios with no turbulence generator within the boundary layer, there wouldn't be an expected large change in turbulence; unless turbulence was onset due to the suction.

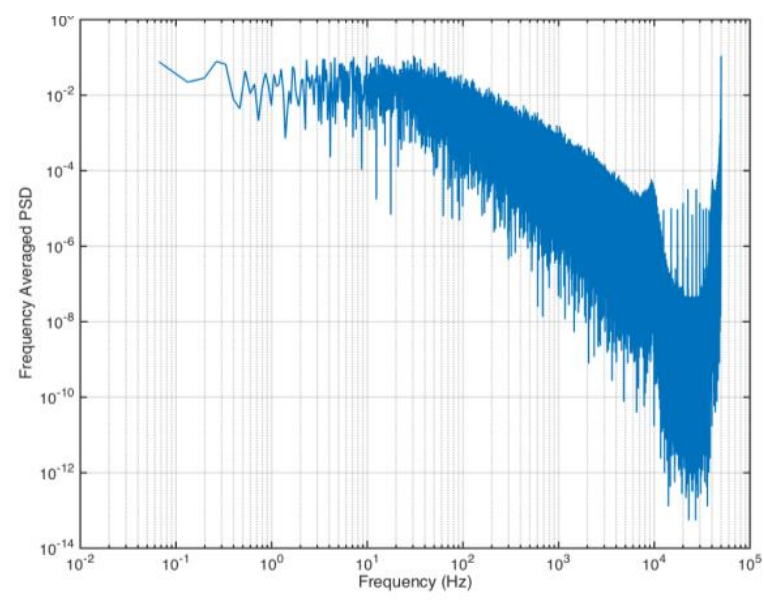

(a)

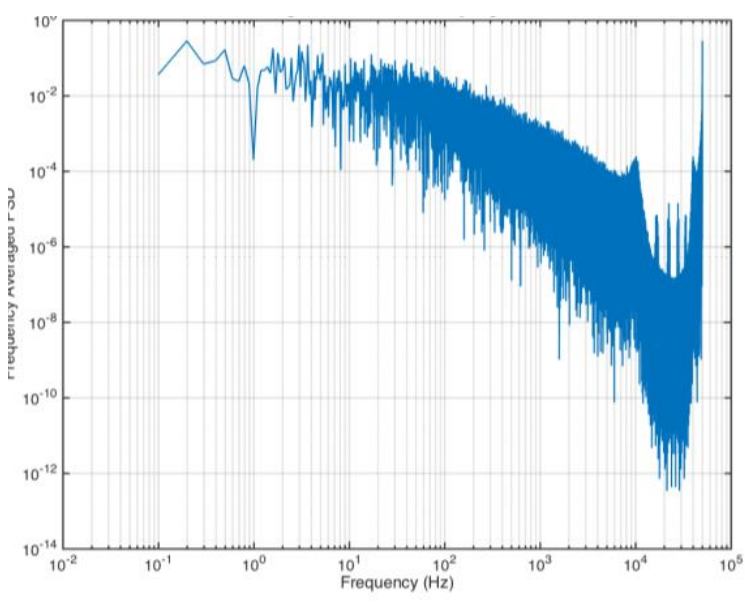

(b)

Figure 50 - Power Spectral Density No Turbulence Trip Inside the Boundary Layer. Suction OFF (a) and Suction ON (b)

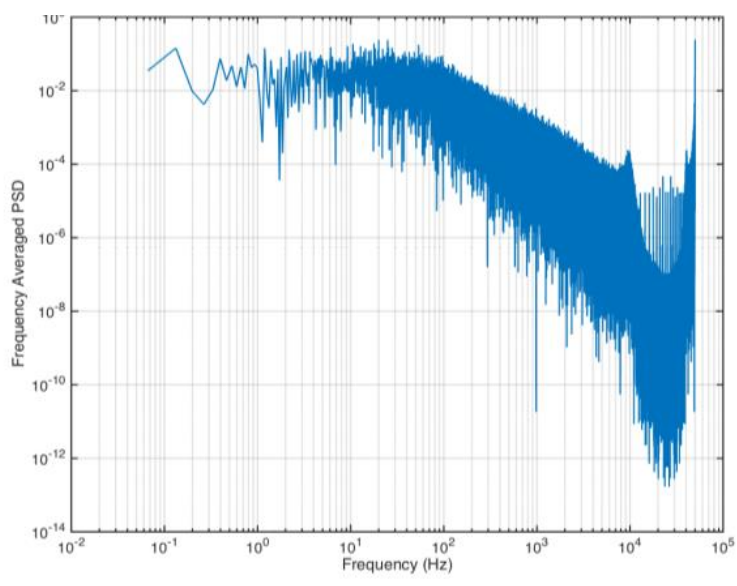

(a)

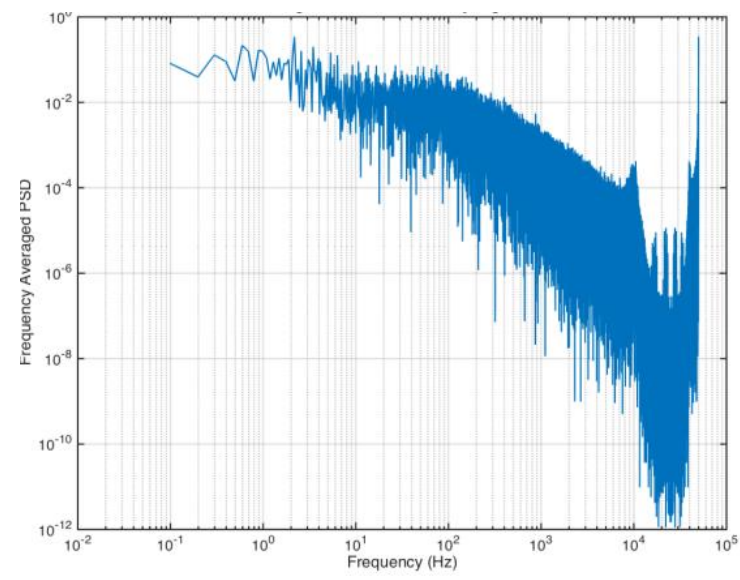

(b)

Figure 51 - Power Spectral Density No Turbulence Trip Near the Boundary Layer Suction. OFF (a) and Suction ON (b) 


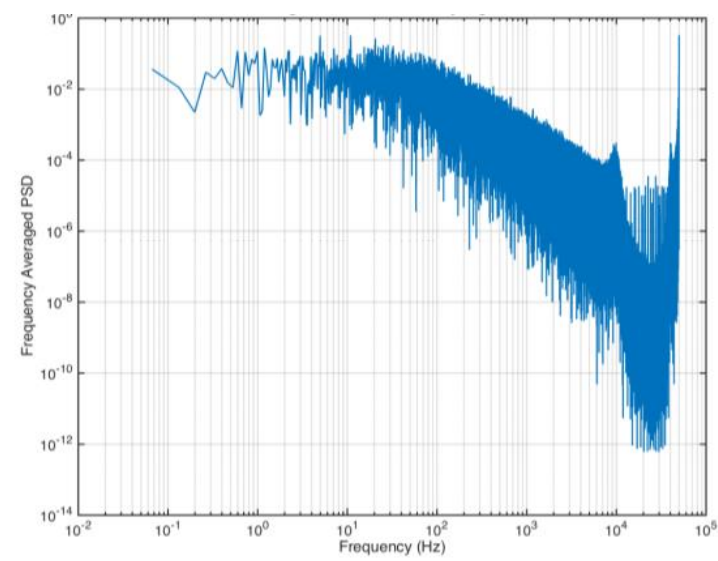

(a)

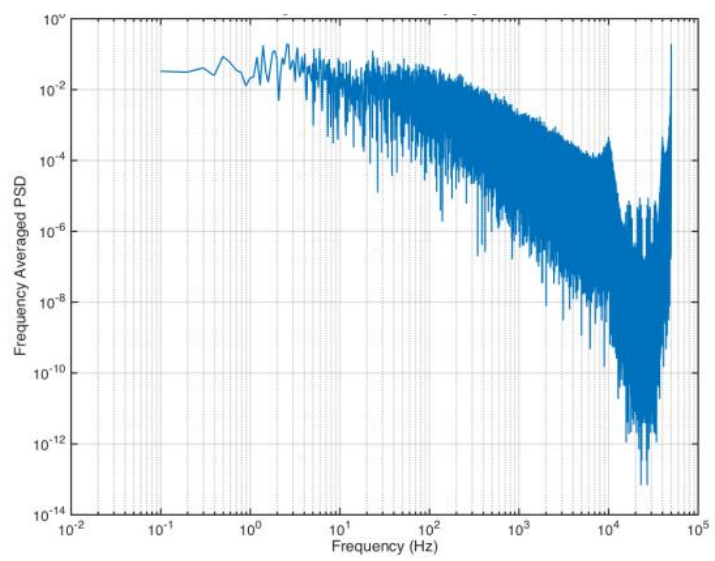

(b)

Figure 52 - Power Spectral Density No Turbulence Trip Outside the Boundary Layer. Suction OFF (a) and Suction ON (b)

Displayed in Figures 50 - 55, the trough and sudden disruption in trend between $10 \mathrm{kHz}$ and $13 \mathrm{kHz}$ can be attributed to the vibration of the hot wire probe support. This vibration of the probe support would be driven by vortex shedding off the probe. These vibration frequencies though again occur in the region where most of the energy has been attenuated and does not affect the calculations of the RMS value.

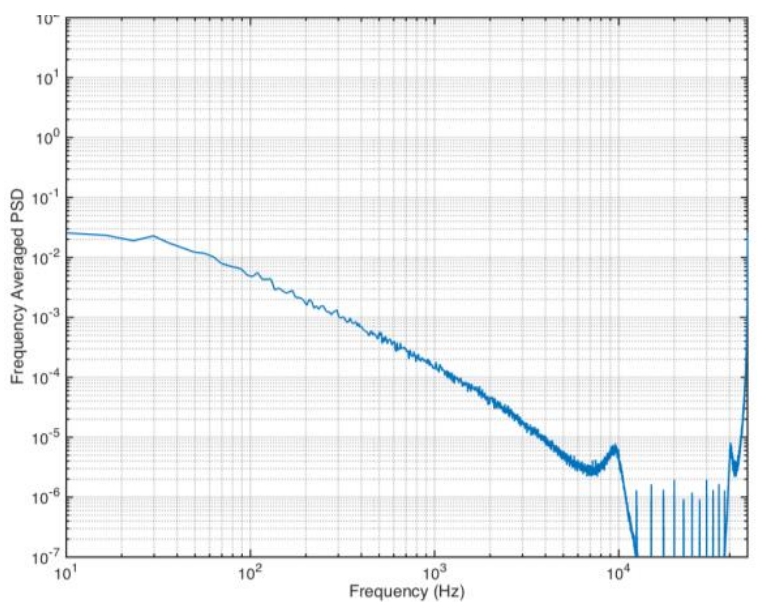

(a)

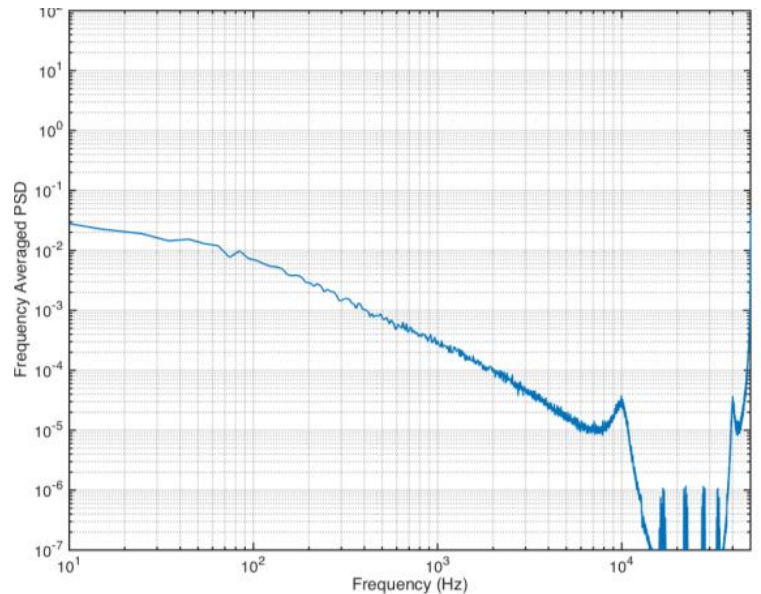

(b)

Figure 53 - Averaged PSD for Scenario of No Turbulence Trip Inside the

Boundary Layer. Suction OFF (a) and Suction ON (b) 


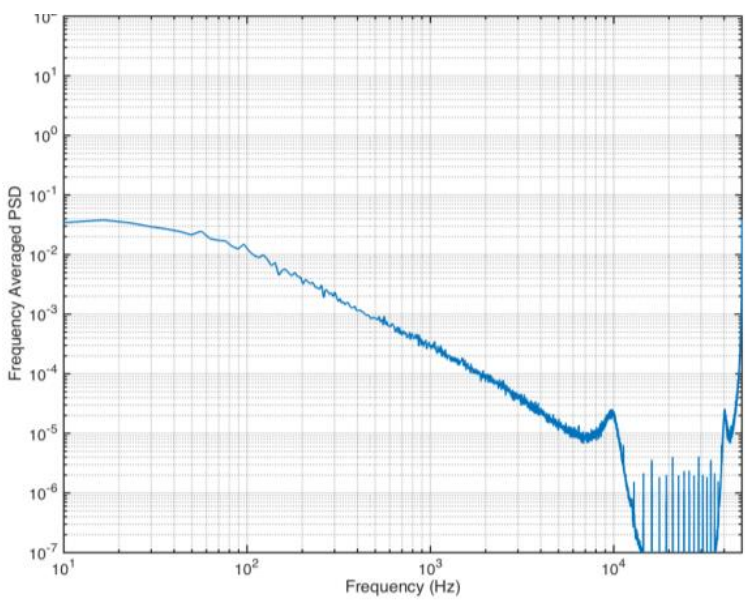

(a)

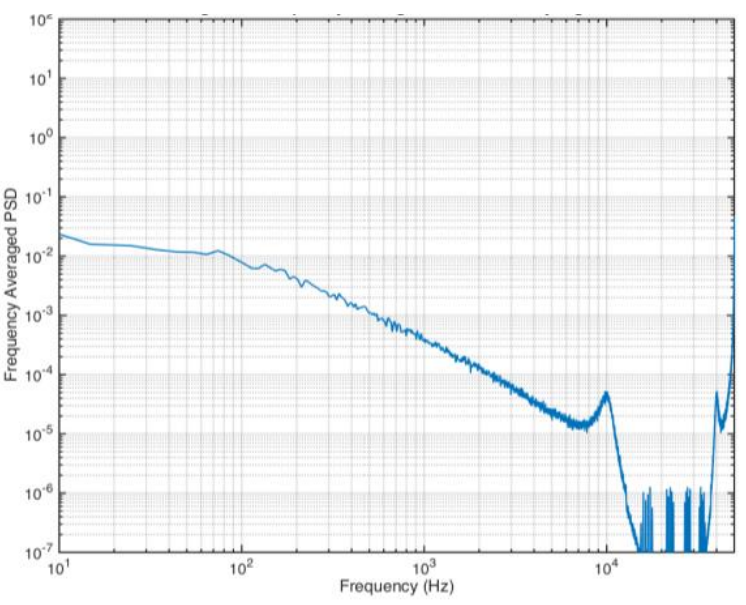

(b)

Figure 54 - Averaged PSD for Scenario of No Turbulence Trip at the

Boundary Layer. Suction OFF (a) and Suction ON (b)

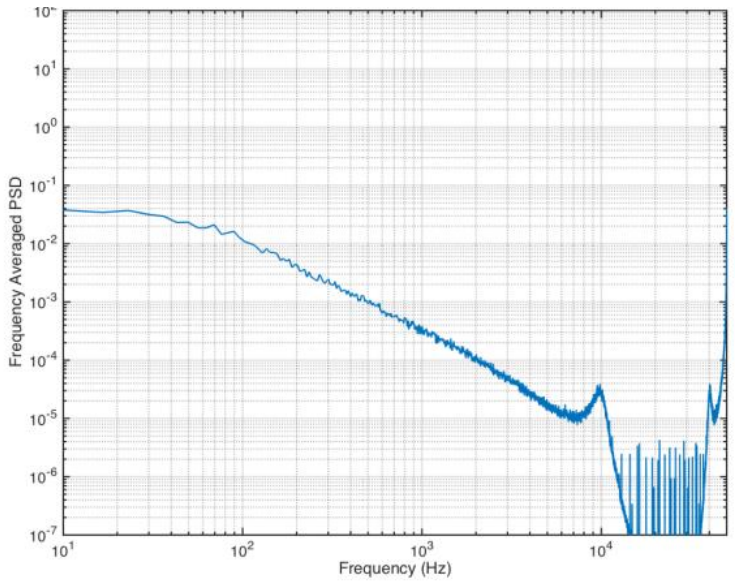

(a)

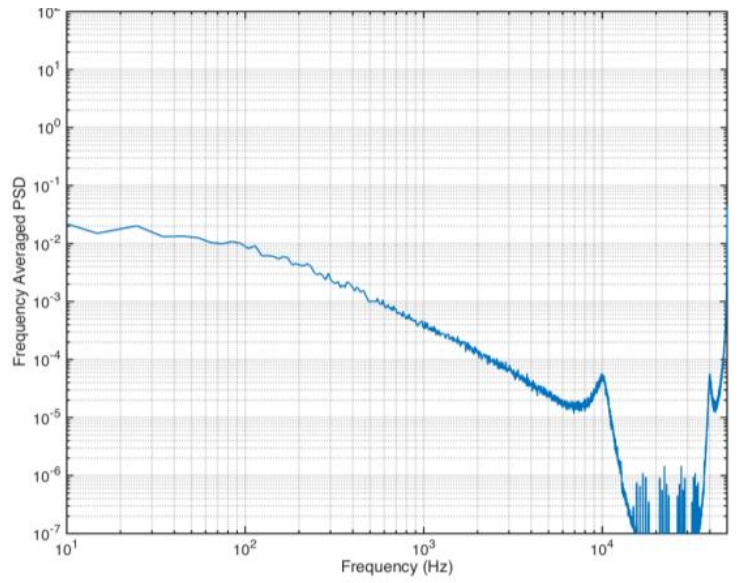

(b)

Figure 55 - Averaged PSD for Scenario of No Turbulence Trip Outside the

Boundary Layer. Suction OFF (a) and Suction ON (b)

Assuming similar mean velocities of two examined flows, the PSD of one velocity signal compared to another PSD flow signal with different turbulent conditions, one can yield which flow has the higher turbulent intensity; and in some cases, which has the larger integral scale. Integrating under the PSD curve of a higher level turbulence case, the $u_{R M S}^{\prime}$ value is higher, therefore identifying which scenario as the higher turbulence intensity. 
Taking the averaged data as shown in Figures 56-58 above, it is then normalized and compared to the Von Karman relation. All the suction scenarios produce similar spectral data from inside the boundary layer to just outside the boundary layer where the turbulence remains but diverge from the Von Karman relation denoted in the red of Figure 56 (b), Figure 57 (b), and Figure 58 (b). In each scenario, there is higher energy at lower frequencies, later attenuating in each scenario approximately at $0.1 \mathrm{kHz}$. It is not completely clear why the values inside the boundary layer with no suction (Figures 54(a) - 56(a)) match clearly to the Von Karman spectrum, contrasted with the scenarios of suction turned on (Figures 54(b) 56(b)) diverge more. This does though confirm though that the suction has an effect on not only the height of the boundary layer but a slight effect to the turbulent state of the boundary layer. Evident in Figure 53 (b) the case of suction on inside the boundary layer, the area under the PSD curve is greater than that for Figure 53 (a) the case of no suction inside the boundary layer.

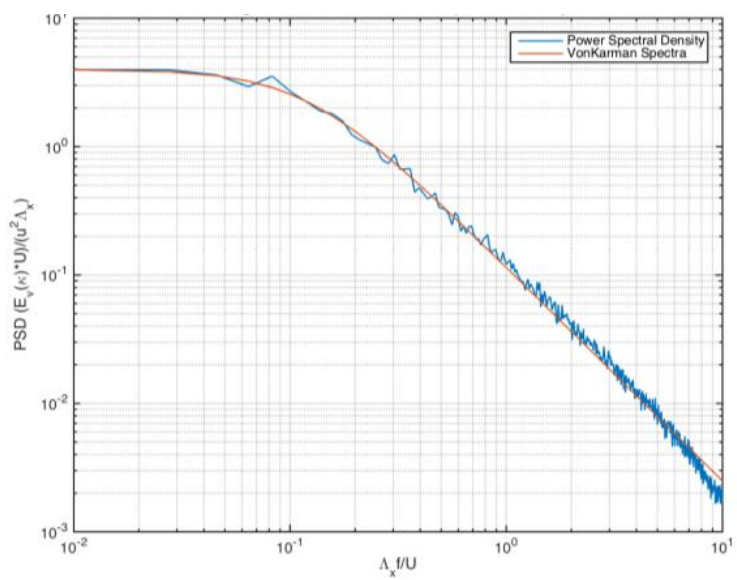

(a)

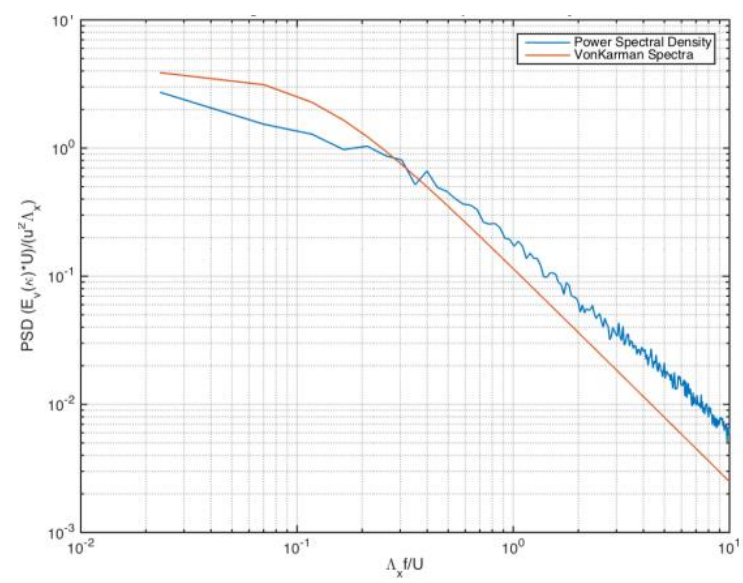

(b)

Figure 56 - Normalized PSD for Scenario of No Turbulence Trip Inside the Boundary Layer Suction OFF (a) and Suction ON (b) 


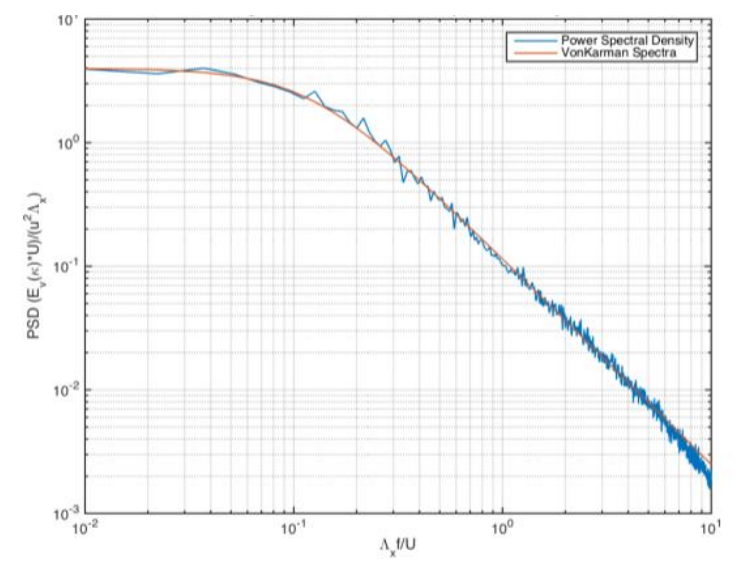

(a)

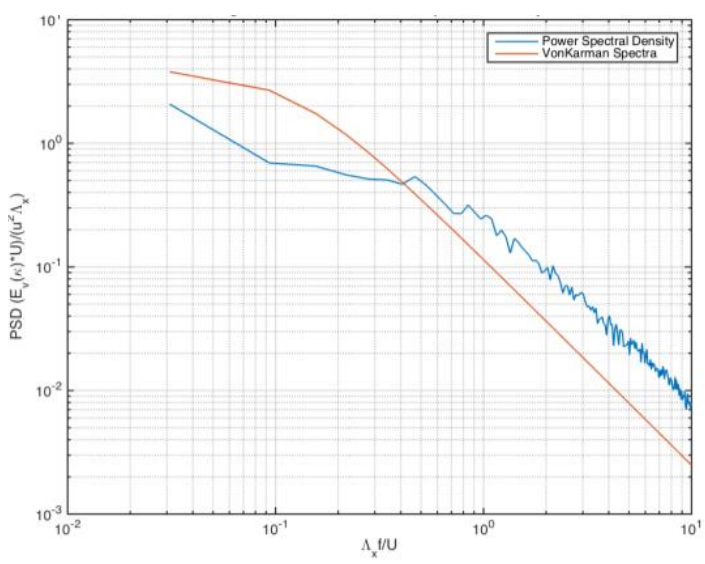

(b)

Figure 57 - Normalized PSD for Scenario of No Turbulence Trip at the Boundary Layer Suction OFF (a) and Suction ON (b)

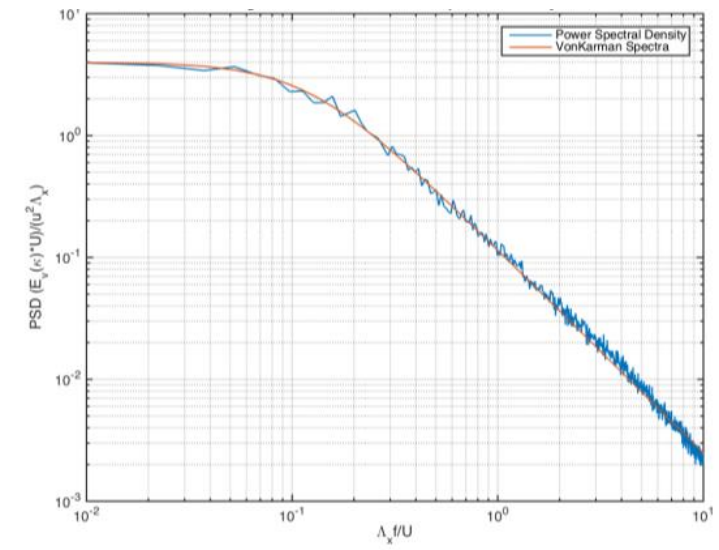

(a)

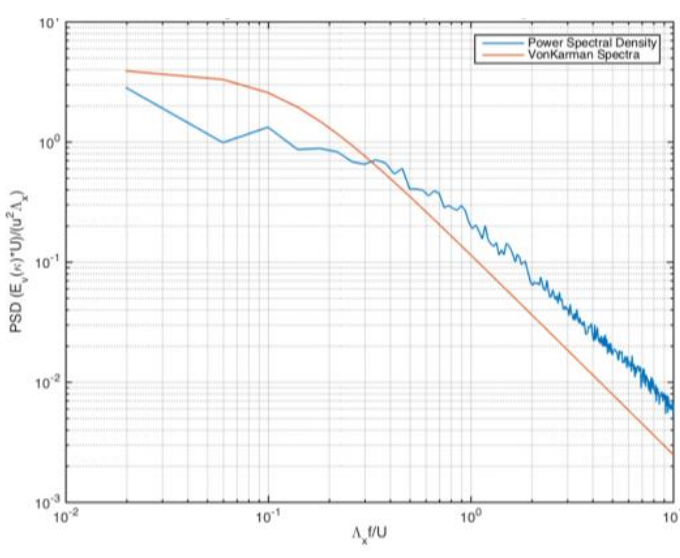

(b)

Figure 58 - Normalized PSD for Scenario of No Turbulence Trip Outside the Boundary Layer Suction OFF (a) and Suction ON (b)

Finally, as several studies during literature review expressed, it is often beneficial to compare the experimental boundary layer data to know theoretical approximations, such as in this case the Blasius solution, depicted in red in Figure 59. This comparison aims to show whether the flow is more laminar than turbulent. In order to do so the variable $\eta$ resenting a non-dimensional parameter called the Blasius similarity variable needs to be defined as: 


$$
\eta=y \sqrt{\frac{U}{2 v x}}
$$

where $\mathrm{U}$, is the freestream velocity, $v$ is the kinematic viscosity, $\mathrm{x}$ is the stream-wise location of the probe, and $y$ is the height perpendicular to the floor.

When adding the suction, the boundary layer remains attached more, maintaining the laminar flow attributes of the boundary layer shown by more closely following the Blasius solution. Figure 59 (b) details this as suction experimental results represented by the blue curve more closely align with the calculated Blasius solution represented by the red curve.

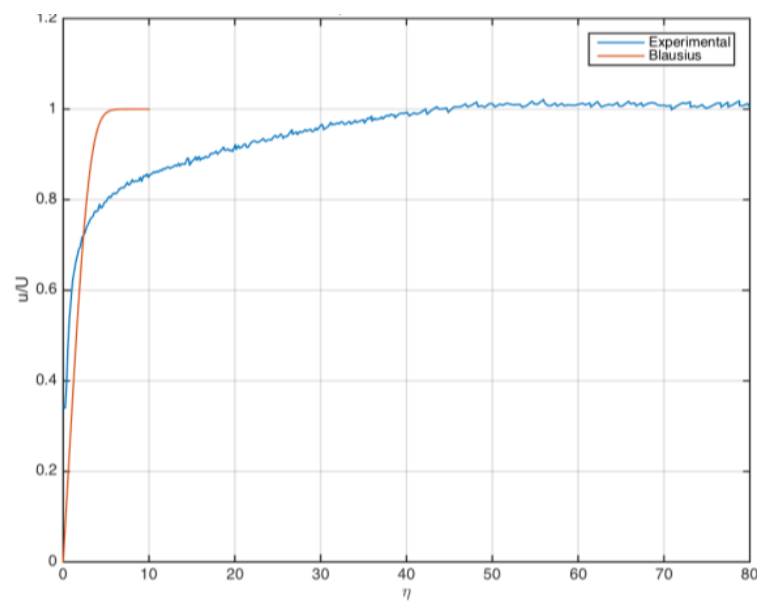

(a)

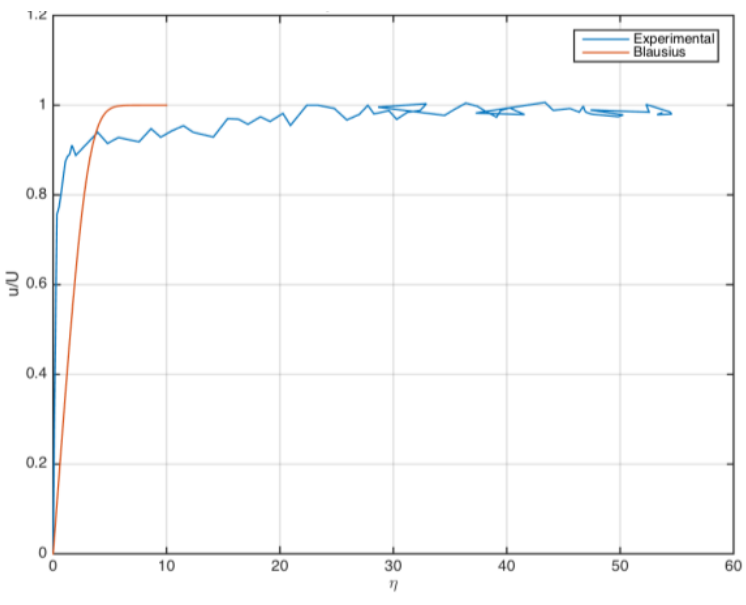

(b)

Figure 59 - Theoretical Blasius comparison to experimental scenario of boundary layer non-tripped flow (a) without suction and (b) with suction

This analysis yields that while the suction did reduce the boundary layer parameters in the scenarios of no turbulence trip inside the boundary layer, the flow inside the boundary layer was already transitional, but not completely turbulent. This is further validated with such an evident turbulent footprint in the data and calculated values (i.e. shape factor values $<2.5$ ), as opposed to a completely discernable laminar profile with such associated characteristics. 


\subsubsection{TRIPPED FLOW}

Table 7 details characteristic values for the tripped boundary layer flow scenarios. The same procedural analysis as for the non-tripped scenarios is also conducted for the tripped turbulent cases detailed below.

Table 7 - Tripped Boundary Layer Flow Results

\begin{tabular}{|c|c|c|}
\hline Property & Turbulence No Suction & Turbulence Suction \\
\hline $\boldsymbol{\delta}$ [in] & 2.25 & 1.17 \\
\hline $\boldsymbol{\delta} / \boldsymbol{D}$ [in] & 4.50 & 2.34 \\
\hline $\boldsymbol{\delta}^{*}[$ in] & 0.947 & .118 \\
\hline $\boldsymbol{\theta}$ [in] & 0.3878 & .092 \\
\hline $\boldsymbol{H}$ & 2.44 & 1.29 \\
\hline $\begin{array}{c}\text { Tu just inside boundary layer } \\
{[\%]}\end{array}$ & 17.8 & 20.2 \\
\hline $\boldsymbol{\Lambda}$ just inside boundary layer & .472 & 2.41 \\
\hline [in] & & \\
\hline
\end{tabular}

The Reynolds number for these cases was approximately $2 \times 10^{5}$. The shape factors shown in Table 7 above yield that the flow is turbulent, however in the case of the turbulence no suction scenario the shape factor varies in a manner that is not consistent with comparison to other data. It is typically assumed that $\mathrm{H}>2.5$ is a laminar flow. While this value of 2.44 is less and turbulent, further inquiry suggests that the flow is turbulent rather. This is mostly likely attributed to the location of the probe relative to the turbulence generator, where due to the presence of a wake, the probe was too far upstream for the turbulent eddies to fully develop into complete formation into turbulent flow. Due to this, the probe picked up spikes in velocity, which when averaged, increased the value of the shape factor. 
The value of $\delta / D$ for the no suction and suction on cases were 4.50 inches and 2.34 inches respectfully The turbulent scenario yields a rather significant increase in boundary layer height and $\delta / D$ as opposed to the non-tripped laminar case. Again, apparent in the tripped scenarios, there is a similarity between all case turbulence intensities, leading to the notion that the boundary layer may not have been in the location as prescribed by the given definition of $99 \%$ freestream conditions. The length scale, as shown in Table 7 , for the suction scenario is 2.41 inches, which again similar to the non-tripped scenarios, is definitely unreasonable and isn't possible for the experiment conducted. More than likely data with the decrease in boundary layer height and the incorrect assumption of the boundary layer location due to the prescribed definition of $99 \%$ freestream conditions, vortices off the passive freestream turbulence bars caused the hotwire probe to erroneously collect values that when post processed further cascaded this unreasonable length scales. Further data collection, comparison, and data post processing needs completed in order to further alleviate such an inaccuracy.

Shown in Figures 60 and 61 are the non-dimensional velocity profiles against the traverse height detailing the boundary layer height of 2.25 inches and 1.17 inches respectively. These figures, similar to the non-tripped scenarios, detail the general shape of the velocity profile traversing up the height of the tunnel. The profile in Figure 60 is possess both parabolic and linear curve attributes in shape, rather than that of a flat bottomed " $U$ " shape as typical with a turbulent velocity profile. Figure 61 better shows this flat bottom "U" shape as it appears to be a better example of a turbulent boundary layer velocity profile. In order to achieve the suction as documented previously by Whitlow, stronger suction is needed, in addition to redefining at what percent of freestream conditions the boundary layer height would be located. 


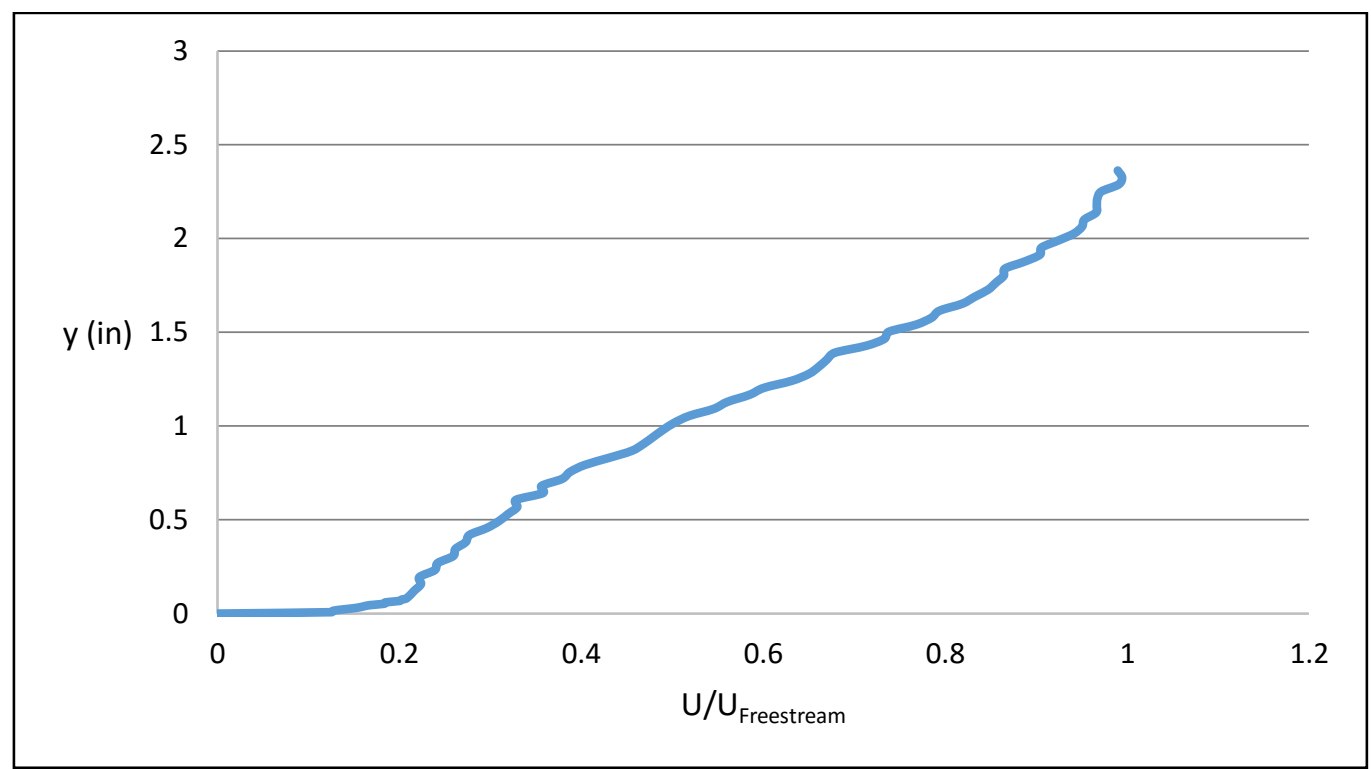

Figure 60 - Traverse Height Velocity Height Turbulence ON and Suction OFF

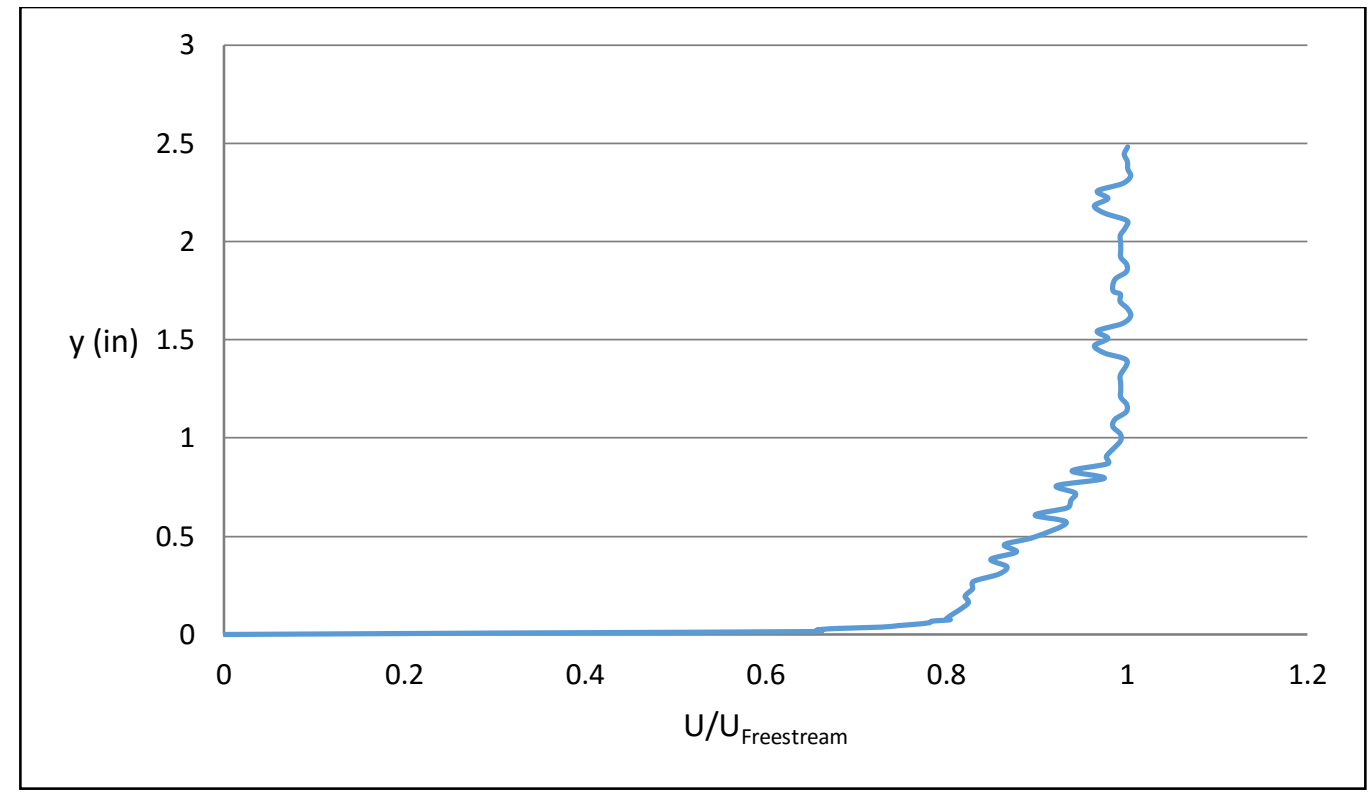

Figure 61 - Traverse Height Velocity Turbulence ON and Suction ON

As similar with the non-tripped boundary layer case, the suction can drastically decrease the boundary layer thickness. In this case, the boundary layer height was decreased by approximately 1 inch. 
No such data with a tripped to turbulent boundary layer flow was completed by Whitlow with the suction slots. However, as these results are in agreement with general trends of what is expected and the non-tripped scenarios, once validated, the data presented can be used to benchmark boundary layer characteristics for future experimental use with the suction slots.

Again by adding the suction, the boundary layer thickness decreases, the boundary layer adhering to the surface more. The length scales for the turbulence models were computed in the same manner as in the non-tripped assumed laminar scenarios, and the autocorrelation is shown below in Figures 62-64 for no suction (a) and suction (b) tripped cases.

The turbulent approximation calculations assume the boundary layer is turbulent from the beginning of the plate. This in actuality is slightly misleading as there is a region of laminar flow, followed by a transition region, and then finally a turbulent region throughout the flow progression. The autocorrelation plots shown in Figures 62-64 are found in the same manner to the first zero crossing, along with the same circumstances and exceptions to values beyond the first zero crossing apply as in the non-tripped laminar boundary layer autocorrelation.

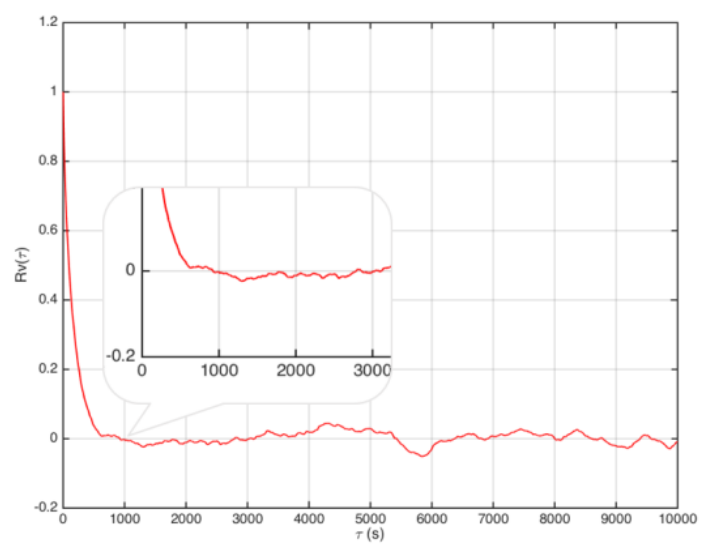

(a)

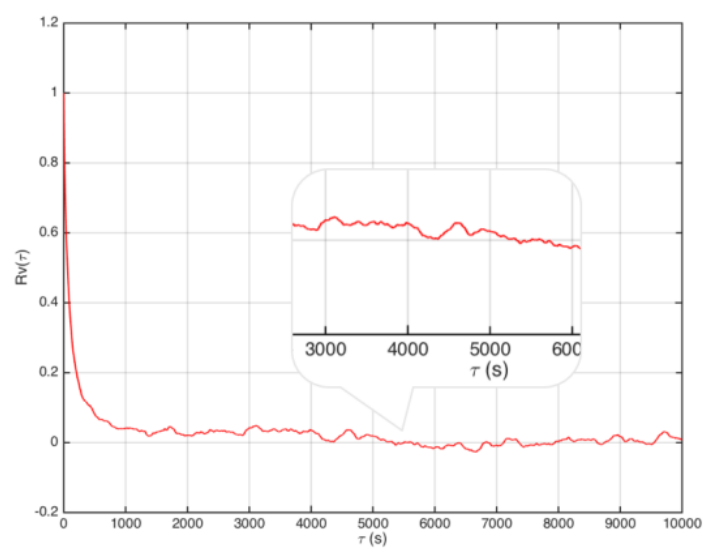

(b)

Figure 62 - Inside the Boundary Layer Autocorrelation Plot with Turbulence Trip for Suction. OFF (a) and Suction ON (b) 


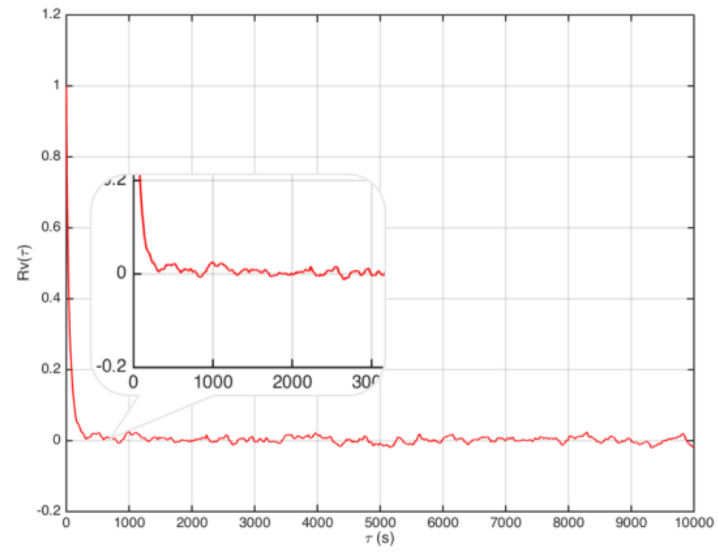

(a)

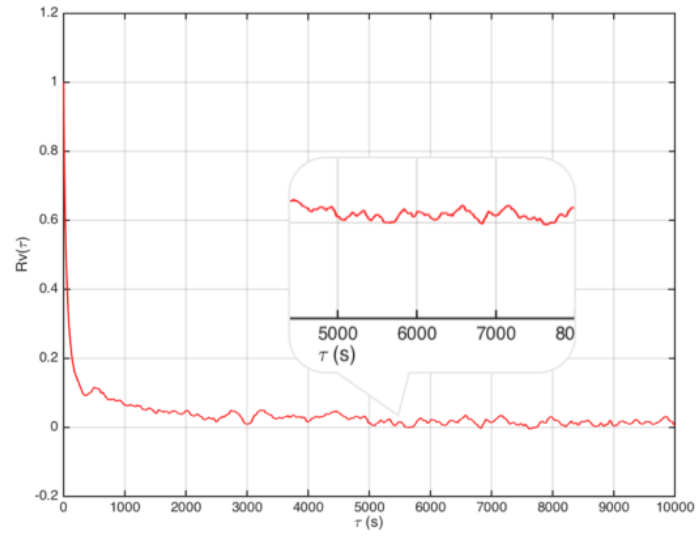

(b)

Figure 63 - At the Boundary Layer Autocorrelation Plot with Turbulence Trip for Suction. OFF (a) and Suction ON (b)

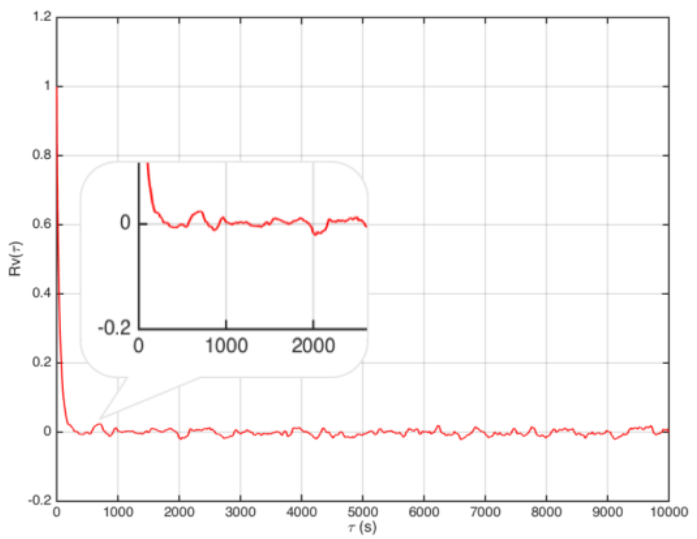

(a)

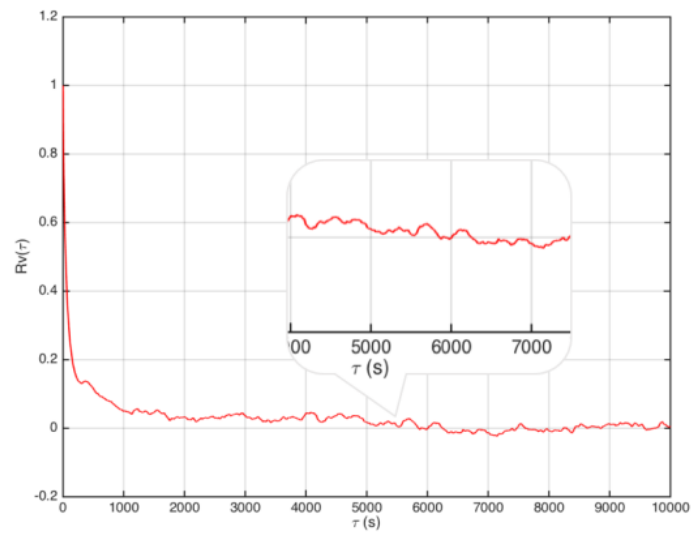

(b)

Figure 64 - Outside the Boundary Layer Autocorrelation Plot with Turbulence Trip for Suction. OFF (a) and Suction ON (b)

The spectral analysis is completed for the scenarios of turbulence being generated by the trip band placed at the end of the suction holes. Figures 65-67 detail the power spectral density of the velocity signals, which was noted as how much kinetic energy is present on 
average at a particular time scale. Figures 68-70 show the averaged PSD representing the generated eddies' average kinetic energy at the respective location.

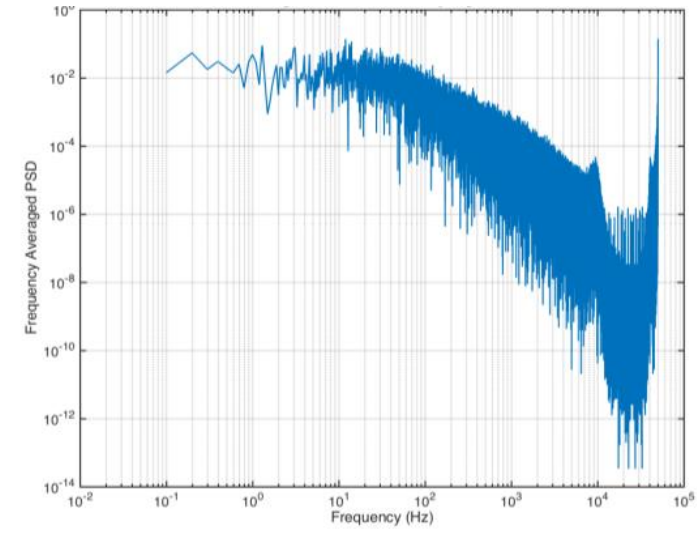

(a)

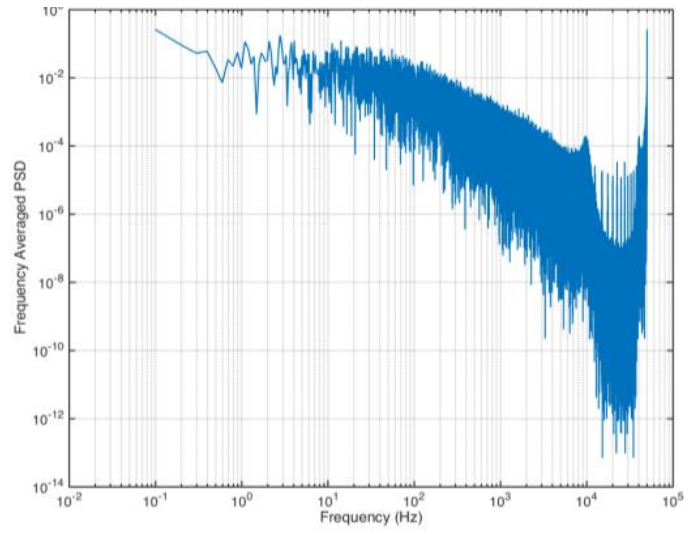

(b)

Figure 65 - Power Spectral Density with Turbulence Trip Inside the Boundary Layer. Suction OFF (a) and Suction ON (b)

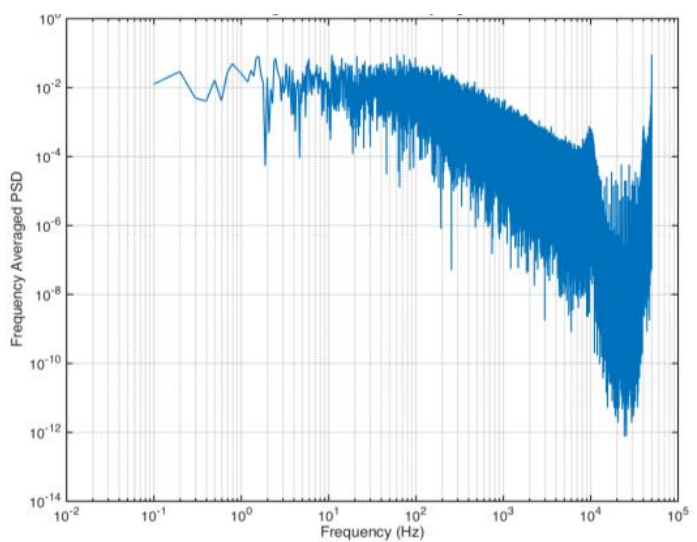

(a)

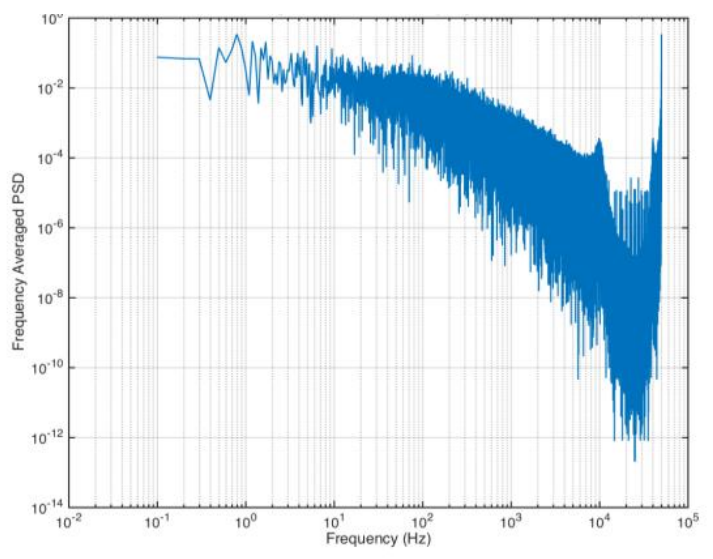

(b)

Figure 66 - Power Spectral Density with Turbulence Trip at the Boundary Layer. Suction OFF (a) and Suction ON (b) 


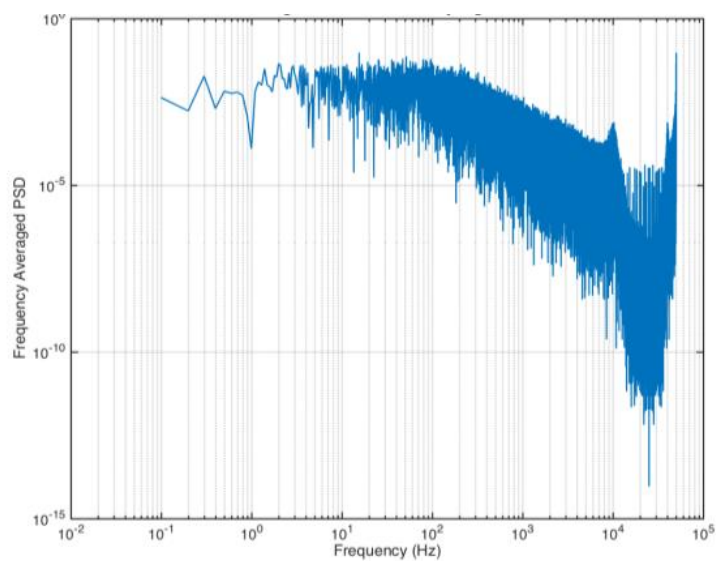

(a)

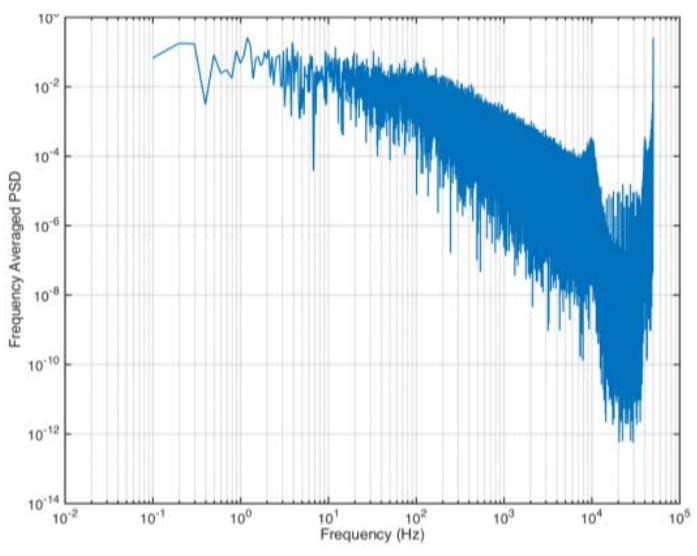

(b)

Figure 67 - Power Spectral Density with Turbulence Trip Outside the Boundary Layer. Suction OFF (a) and Suction ON (b)

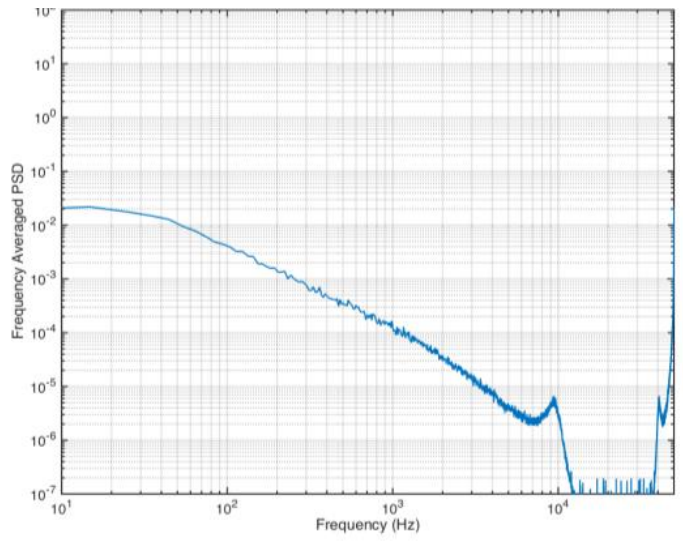

(a)

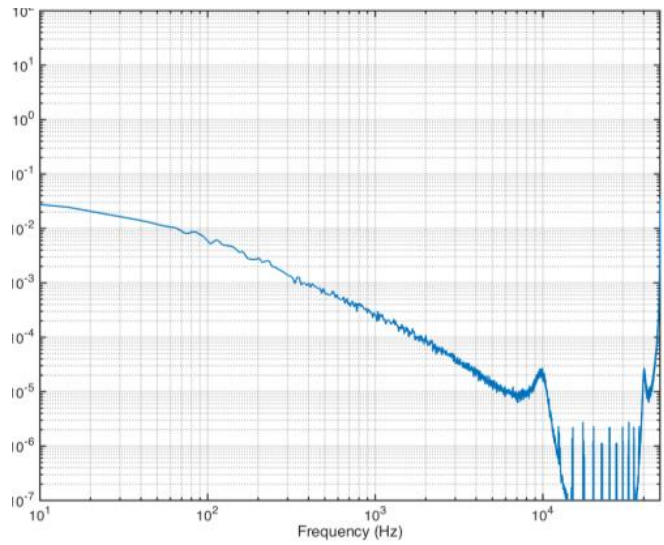

(b)

Figure 68 - Averaged PSD for Scenario with Turbulence Trip Inside the

Boundary Layer. Suction OFF (a) and Suction ON (b) 


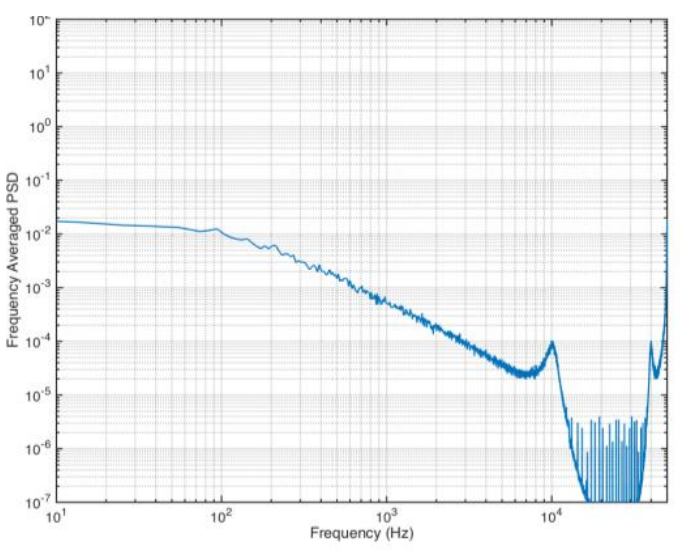

(a)

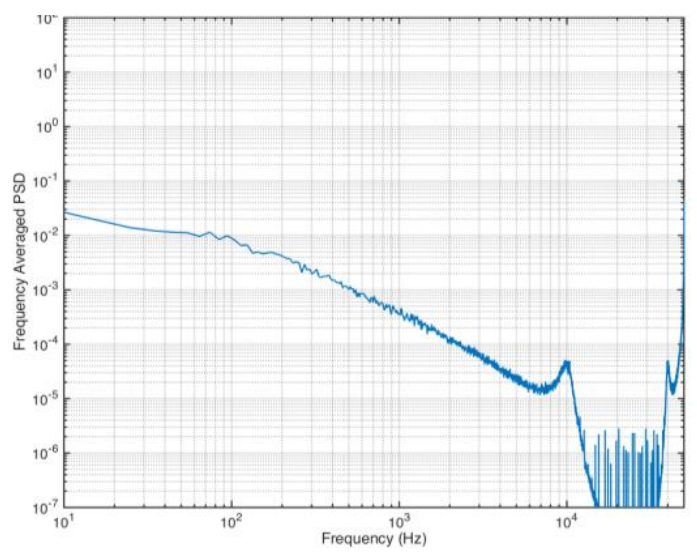

(b)

Figure 69 - Averaged PSD for Scenario with Turbulence Trip at the Boundary Layer. Suction OFF (a) and Suction ON (b)

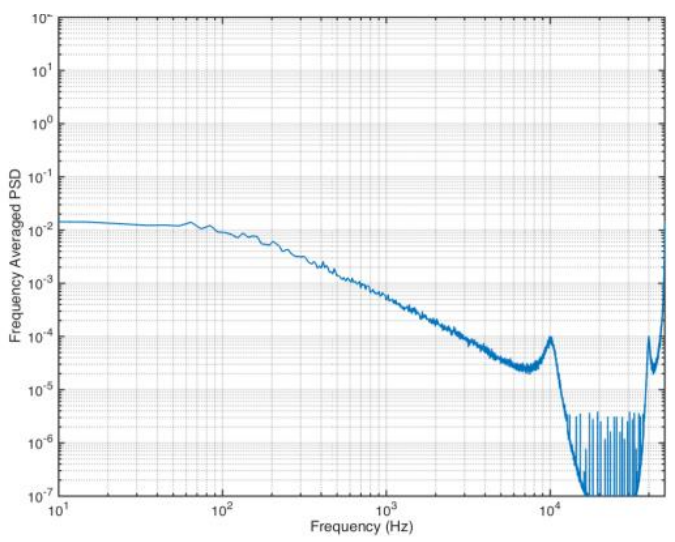

(a)

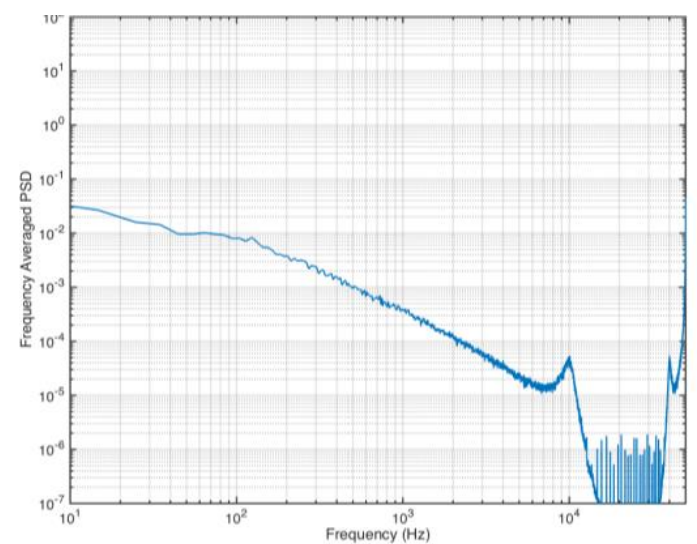

(b)

Figure 70 - Averaged PSD for Scenario with Turbulence Trip Outside the Boundary Layer. Suction OFF (a) and Suction ON (b)

The turbulent energy matches more closely to the theoretical values with the suction off in these last two tests signifying that the boundary layer is in fact turbulent, however upon reattaching the flow with suction, some of the turbulent effects are smaller. At frequencies between $10 \mathrm{kHz}-13 \mathrm{kHz}$ there is a change in trend with a sudden disturbance similar to the non-tripped laminar scenarios. This as previously described, this is attributed to the vibration of the probe support. 


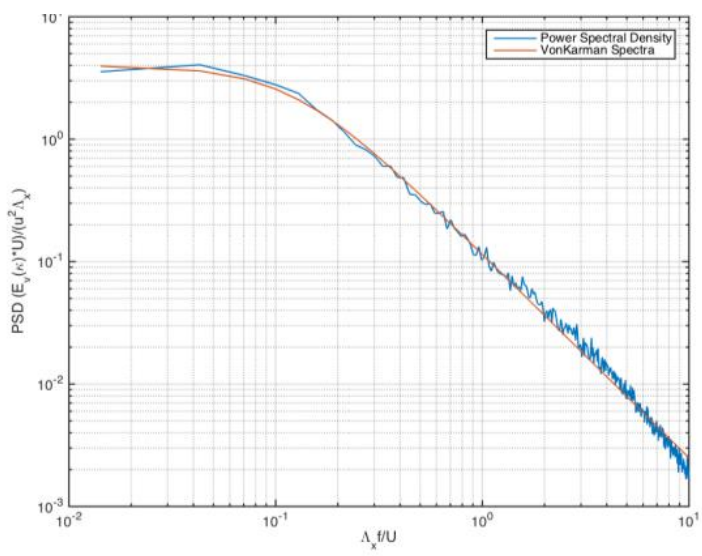

(a)

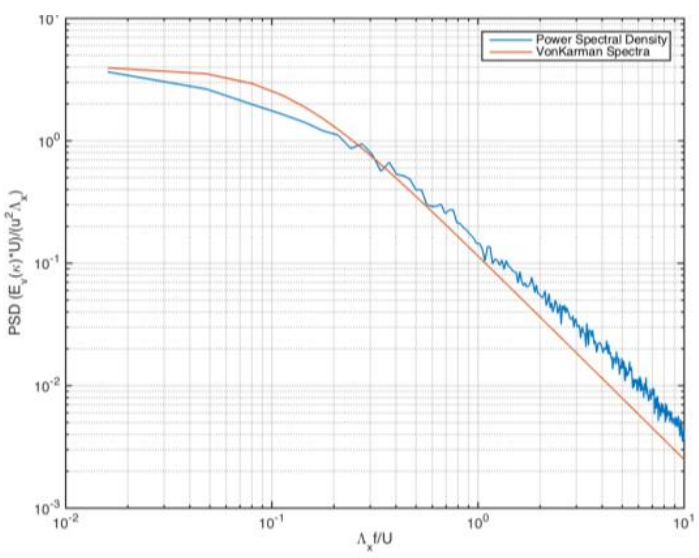

(b)

Figure 71 - Normalized PSD for Scenario with Turbulence Trip Inside the Boundary Layer Suction OFF (a) and Suction ON (b)

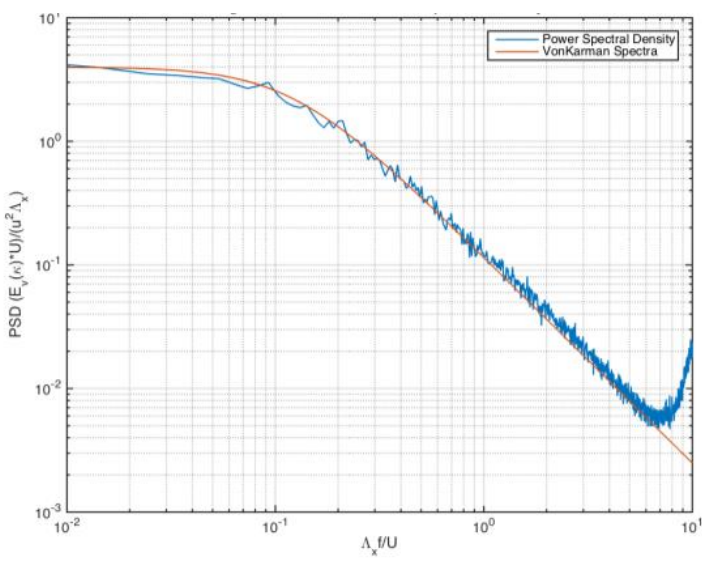

(a)

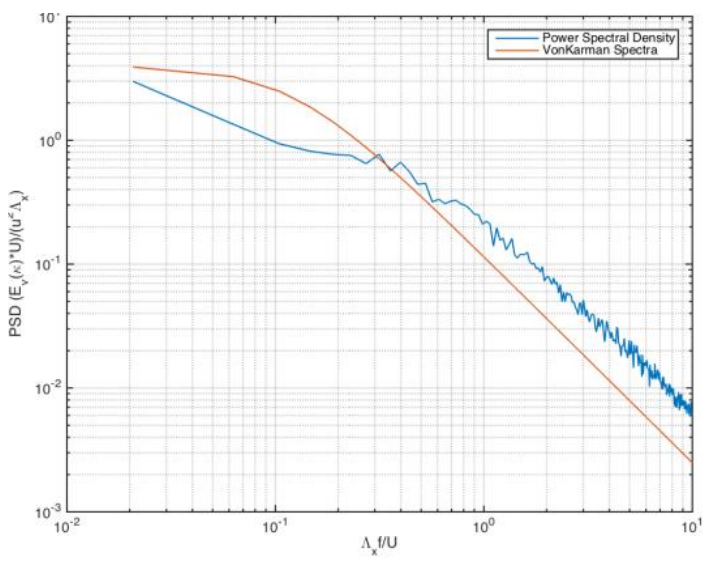

(b)

Figure 72 - Normalized PSD for Scenario with Turbulence Trip at the Boundary Layer Suction OFF (a) and Suction ON (b) 


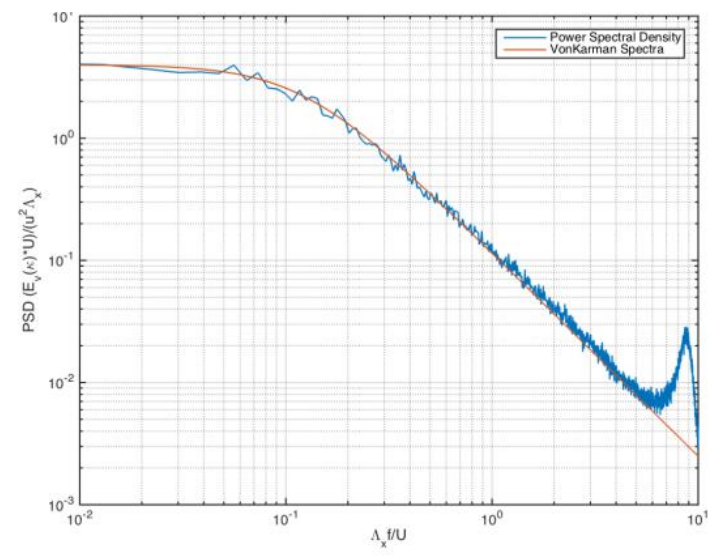

(a)

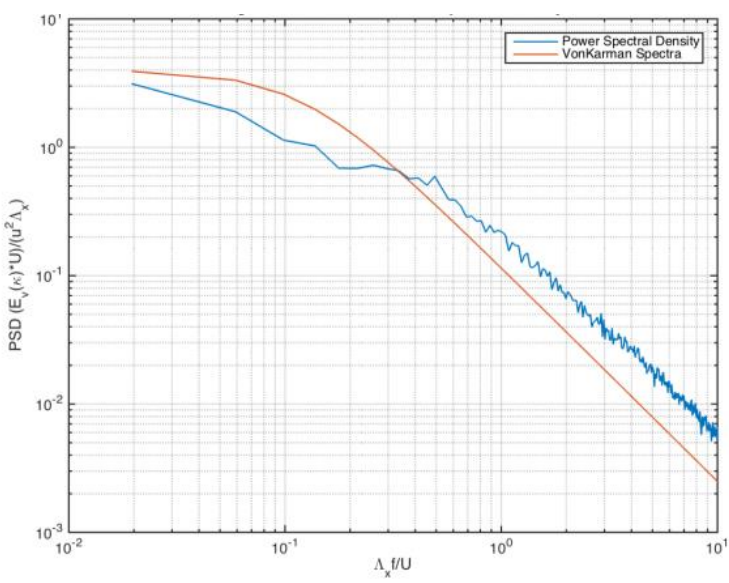

(b)

Figure 73 - Normalized PSD for Scenario with Turbulence Trip Outside the Boundary Layer Suction OFF (a) and Suction ON (b)

Figures 71-73 detailing the normalized power spectral density signal align rather closely with the VonKarman Spectra except when the suction is turned on for the three presented locations. It is believed that this deviation is somehow contributed to the cascading error in data collection of the suction on cases followed by post processing that erroneous data.

As expected and shown in Figure 74, the experimental data does not match closely with Blasius solution. The Blasius solution represents laminar flow and does not accurately depict turbulence. For the case of turbulence and no suction considered (Figure 74 (a)); while this value shouldn't be similar to Blasius, the shape would be expected to look more like that of Figure 74 (b). This, again, is where the normalized spectral data is useful signifying the turbulent energy contained in the eddies shown through the length scales. Further testing is needed to fully understand what impact of why the data resolves to the trend expressed in Figure 74 (a). 


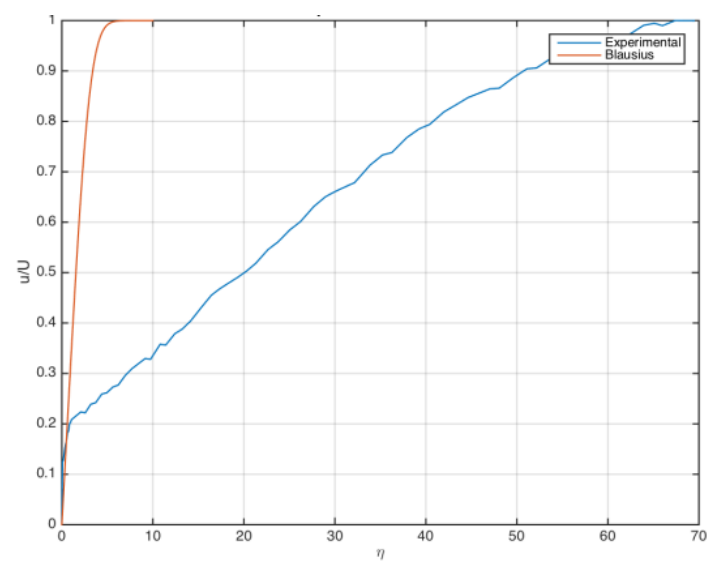

(a)

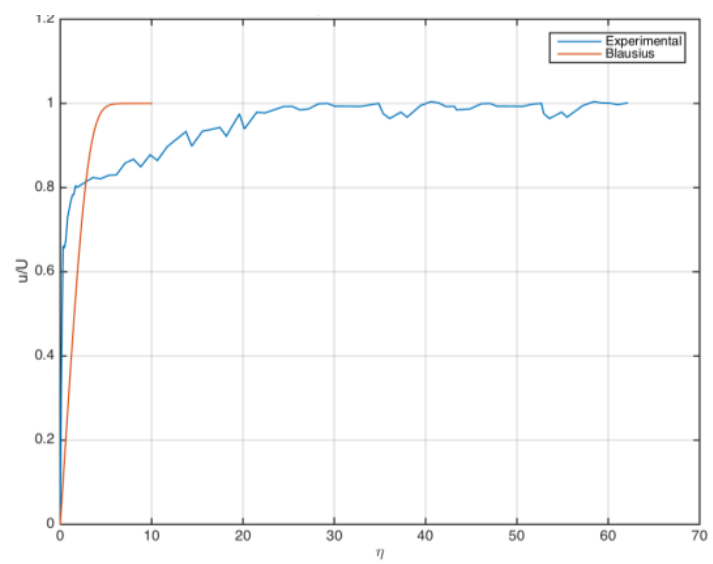

(b)

Figure 74 - Theoretical Blasius comparison to experimental scenario of boundary layer tripped flow (a) without suction and (b) with suction 


\section{CHATPER 6: CONCLUSIONS AND FUTURE WORK}

\subsection{CONCLUSIONS}

The WVU Turbine Aerodynamic and Advanced Cooling wind tunnel facility was previously idle, and upon successful reinstatement and relocation of the facility, a characterization of the boundary layer and active suction system, along with a full instrumentation calibration, were completed and compared to previous benchmarking documentation. The author rebuilt this subsonic, low-temperature wind tunnel facility, which is designed to non-dimensionally investigate film cooling effectiveness. The facility originally designed and reconstructed with interchangeable components allows flexibility for modifications in the setup for additional measurement techniques and turbine cooling schemes. Part of this flexibility is through interchangeable cooling hole plates, containing various cooling hole geometries, interchangeable passive freestream turbulence bars, and an active boundary layer suction system. This investigation sought to experimentally review the influence of the boundary layer suction slots and turbulence which the cooling hole plate would experience downstream. In order to achieve this boundary layer characterization data was needed.

Upon finding the characteristic data such as the displacement thickness, momentum thickness etc., for each testing case, turbulence was then examined to reveal additional considerations which can be used in future experiments. As demonstrated, by adding suction, the boundary layer thickness decreases from 1 inch to roughly 0.8 inch, an almost 0.25 -inch decrease. Again, the data suggests that while initially proposed as laminar and non-tripped boundary layers, the flow has properties with minimal turbulent characteristics closer appointed as transitional flow. With this notion, it would then be expected that some laminar attributed would appear with the PSD showing turbulent characteristics. The scenarios with a turbulence trip designed to ensure the boundary layer turbulence achieved full turbulence since both the velocity profile and PSD conclude that boundary layer properties are that of a turbulent nature. Additionally, because a strict adherence to the definition of a boundary layer as $99 \%$ freestream the actual data may be misleading. This is not terms to say the data is 
invalid, simply that the actual boundary layer may actually exist at a lower percent of freestream conditions. Standard convention is documents that the boundary layer is at $99 \%$ freestream conditions, however this is more an established baseline. Should the investigation be reconducted with this consideration, non-tripped data would be expected to match Whitlow experimental data very closely.

Utilizing the suction greatly affected the boundary layer parameters regardless of the boundary layer state. Although, further investigation should commence for supplemental insight in order to fully quantify the extent of suction influence. This would include being able to vary the suction and perform the testing at various $\mathrm{x}$ locations progressing from the test section inlet. The spectral data assisted was useful to quantify the turbulence experienced inside and at the boundary layers. One main point to retrieve from this study and from prior Whitlow data is that the suction needs to be increased for further ability to match nondimensional parameters. Part of ongoing work is to change this current Radon fan and associated setup to another fan capable of drawing more low momentum fluid from the test section floor. A noted possible alternative is a "squirrel cage" centrifugal fan which could allow this increase in suction. This would then require testing as conducted for this experiment to be recompleted, and the boundary layer characterized again.

The primary concern of the facility is aimed at film cooling effectiveness for gas turbine blades located in the few first stages of the high pressure turbine section of gas turbine engines. This investigation therefore proposed it advantageous to characterize the boundary layers and demonstrate boundary layer influencing techniques in order to completely understand the influence of boundary layers similar to what would be present in blade film cooling schemes. The purpose of including a boundary layer suction mechanism then has actual application in the ability to match operating blade conditions. Replication of aerodynamic conditions such as the Reynolds number is possible in the WVU TAAC facility, but it is not realistically able to match the Mach number. In this reason, boundary layer suction is crucial to understand since the tunnel will produce a boundary layer not representative of conditions inside an actual engine. Additionally, the ratio of boundary layer 
thickness to cooling hole diameter will need to be matched. Experimentally finding the impact of decreasing the boundary layer by as much as 1 inch in the mentioned cases, this data will be useful in selecting a suction fan or suction metering value that produces a desired boundary layer thickness to cooling hole diameter ratio.

These statements expose that the thermodynamic design of hot section turbine engine components will profit from the data on film cooling effectiveness of improved film cooling hole designs for a variety of turbine applications. The benefits of this investigation will lead to research which can have a significant influence in today's energy generation and aircraft propulsion. This then contributes to a substantial socioeconomic impact with an evergrowing energy consumption and demand for airline commerce. Improving the film cooling effectiveness can increase the performance and turbine inlet temperature leading to increases in engine efficiencies. Such a small increase in efficiency will reduce hazardous emissions for both land-based applications and the transportation industry. This thereby solves a critical demand for energy in an environmentally conscious manner. It will be through this increase in efficiency that costs associated with implementation and maintenance can be reduced, saving various programs and consumers significantly in the long-term outlook.

\subsection{FUTURE WORK}

The results of this investigation yield an understanding of suction slot influence on boundary layer characteristics while also establishing the recommissioned WVU tunnel benchmarks for future investigations pertaining to gas turbine cooling schemes. This investigation establishes a good starting point, but clearly reveals that additional work is necessary to understand the effect of boundary layer thickness on film cooling effectiveness and the convective heat transfer coefficient by implementing the ability to vary the amount of suction in the suction slots. Once the wind tunnel facility is completely back online, it is desired to electronically control the Radon suction fan electronically via Labview ${ }^{\circledR}$ or MATLAB ${ }^{\circledR}$ code, and further document boundary layer characteristics. Further capabilities of this facility include testing other film cooling techniques such as combustor lining cooling, 
contoured end wall cooling, and cooling existing for pulse detonation turbine engines with minor facility modifications.

Currently discussed future work within the wind tunnel facility include implementing infrared thermography in supplement to LDV or PIV systems. Additionally, due to the design of the wind tunnel, various other passive grid systems can be manufactured and implemented to vary the freestream turbulence intensities, or by implementing an actively blown turbulent grid system. With the possibility of interchanging the cooling hole plate located as the floor in part of the test section, further complex hole geometries can be pursued to continue existing studies conducted in the facility. This is greatly beneficial as more complex blades can now be placed into production with the continuing development of additive manufacturing. 


\section{REFERENCES}

[1] J. Escobar, "Turbine Engine History | AviationPros.com," AVIATION PROS, 2006. [Online]. Available: http://www.aviationpros.com/article/10383708/turbine-enginehistory. [Accessed: 16-Jun-2017].

[2] F. Landis, "gas-turbine engine | Britannica.com," Britannica Encyclopedia, 2008. [Online]. Available: https://www.britannica.com/technology/gas-turbineengine\#ref134512. [Accessed: 21-Jun-2017].

[3] Encyclopedia.com, "Sir Frank Whittle facts, information, pictures | Encyclopedia.com articles about Sir Frank Whittle," Encyclopedia of World Biography. Encyclopedia.com, 2004.

[4] MUIB, "Made Up in Britain: Jet Engine : Sir Frank Whittle 1930," British Inventions Discoveries Creations and Innovations, 2017. [Online]. Available: http://madeupinbritain.uk/Jet_Engine. [Accessed: 21-Jun-2017].

[5] "The Development of Jet Engines During The War," Standford University, 2004. [Online]. Available: https://cs.stanford.edu/people/eroberts/courses/ww2/projects/jetairplanes/planes.html. [Accessed: 21-Jun-2017].

[6] N. Hall, "Turbine Engine Thermodynamic Cycle - Brayton Cycle," NASA, 2015. [Online]. Available: https://www.grc.nasa.gov/www/k-12/airplane/brayton.html. [Accessed: 21-Jun-2017].

[7] L. Langston, "Introduction to Gas Turbines for Non- Engineers," Glob. Gas Turbine News, vol. 37, no. 2, p. 7, 1997.

[8] S. Farokhi, Aircraft propulsion. 2014.

[9] S. Andrew Hayes, A. C. Nix, C. M. John Kuhlman, and W. W. Huebsch, "An Experimental Investigation On the Effects of Freestream Turbulence Intensity On Film Cooling Effectiveness and Heat Transfer Coefficient for an Anti-Vortex Hole," 2014.

[10] K. Bradshaw, "Ramp Ramblings 4 | The Duxford Aviation Society," Duxford Aviation Society, 2015. [Online]. Available: http://das.org.uk/ramp-ramblings-4/. [Accessed: 22-Jun-2017].

[11] C. Gonzalez, "What's the Difference Between Turbine Engines?," Machine Design, 2016. [Online]. Available: http://www.machinedesign.com/motorsdrives/what-sdifference-between-turbine-engines. [Accessed: 22-Jun-2017].

[12] D. G. Bogard and K. a Thole, "Gas Turbine Film Cooling," J. Propuls. Power, vol. 21:6, no. December, p. 24, 2005.

[13] H. Saravanamuttoo, G. Rogers, H. Cohen, and P. Straznicky, "The Cooled Turbine," in Gas Turbine Theory, Six., Essex, England, United Kingdom: Pearson Education Limited, 2009, pp. 366-382.

[14] A. C. Nix, B. Andrew Carl Nix, T. E. Diller, C.-C. F. Wing Ng, and C.-C. A. Karen 
Thole Joseph A Schetz Clinton L Dancey, "Effects of High Intensity, Large-Scale Freestream Combustor Turbulence On Heat Transfer in Transonic Turbine Blades," Virginia Polytechnic Institute and State University, 2003.

[15] J.-C. Han And S. Dutta, "Recent Developments in Turbine Blade Internal Cooling," Ann. N. Y. Acad. Sci., vol. 934, no. 1, pp. 162-178, Jan. 2006.

[16] N. Zuckerman and N. Lior, "Jet impingement heat transfer: Physics, correlations, and numerical modeling," Advances in Heat Transfer, vol. 39, no. C. pp. 565-631, 2006.

[17] R. Brakmann, L. Chen, B. Weigand, and M. Crawford, "Experimental and Numerical Heat Transfer Investigation of an Impinging Jet Array on a Target Plate Roughened by Cubic Micro Pin Fins 1," J. Turbomach., vol. 138, no. 11, p. 111010, Jun. 2016.

[18] A. R. Al Ali and I. Janajreh, "Numerical Simulation of Turbine Blade Cooling via Jet Impingement," Energy Procedia, vol. 75, pp. 3220-3229, Aug. 2015.

[19] T. G. Lewis, R. P. Darken, T. Mackin, and D. Dudenhoeffer, "Model-based risk analysis for critical infrastructures," Crit. Infrastruct. Secur. Assessment, Prev. Detect. Response, vol. 54, pp. 3-19, 2012.

[20] J.-C. Han and L. M. Wright, "4.2.2.2 Enhanced Internal Cooling of Turbine Blades and Vanes," NETL Gas Turbine Handb., pp. 321-352, 2206.

[21] N. A. Cumpsty and A. (Andrew L. . Heyes, Jet propulsion: a simple guide to the aerodynamics and thermodynamic design and performance of jet engines. Cambridge University Press, 2015.

[22] B. Lakshminarayana, Fluid Dynamics and Heat Transfer of Turbomachinery. Hoboken, NJ, USA: John Wiley \& Sons, Inc., 1995.

[23] S. A. Hayes, A. C. Nix, C. M. Nestor, D. T. Billups, and S. M. Haught, "Experimental investigation of the influence of freestream turbulence on an anti-vortex film cooling hole," 2017.

[24] K. Kadotani and R. J. Goldstein, "On the Nature of Jets Entering A Turbulent Flow: Part B-Film Cooling Performance," J. Eng. Power, vol. 101, no. 3, p. 466, Jul. 1979.

[25] K. Kadotani and R. J. Goldstein, "On the Nature of Jets Entering A Turbulent Flow: Part B-Film Cooling Performance," J. Eng. Power, vol. 101, no. 3, p. 466, Jul. 1979.

[26] J. C. Han and A. B. Mehendale, "Flat-Plate Film Cooling With Steam Injection Through One Row and Two Rows of Inclined Holes," J. Turbomach., vol. 108, no. 1, p. 137, Jul. 1986.

[27] B. A. Haven, D. K. Yamagata, M. Kurosaka, S. Yamawaki, and T. Maya, "AntiKidney Pair of Vortices in Shaped Holes and Their Influence on Film Cooling Effectiveness," in Volume 3: Heat Transfer; Electric Power; Industrial and Cogeneration, 1997, p. V003T09A007.

[28] T. J. Juliano, P. Kumar, D. Peng, J. W. Gregory, J. Crafton, and S. Fonov, "Single- 
shot, lifetime-based pressure-sensitive paint for rotating blades," Meas. Sci. Technol., vol. 22, no. 8, p. 85403, Aug. 2011.

[29] T. Liu and J. P. Sullivan, Pressure and Temperature Sensitive Paints. Springer, 2005.

[30] K. Azar, "Introduction to Liquid Crystal Thermography."

[31] R. Hughes, L. Castoro, L. Kiefert, and H.-P. K. Nyunt, "FTIR in Gem Testing • FTIR Intrigue • Lotus Gemology," Lotus Gemology, 2017. [Online]. Available: http://www.lotusgemology.com/index.php/library/articles/294-ftir-in-gem-testingftir-intrigue-lotus-gemology. [Accessed: 06-Jul-2017].

[32] B. R. Munson, T. H. (Theodore H. Okiishi, W. W. Huebsch, and A. P. Rothmayer, Fundamentals Of Fluid Mechanics. John Wiley \& Sons, Inc, 2013.

[33] A. Bejan, Convection Heat Transfer. 2013.

[34] J. Mathieu and J. Scott, An Introduction To Turbulent Flow. Cambridge University Press, 2000.

[35] A. Dewan, Tackling turbulent flows in engineering. Springer, 2011.

[36] R. Groh, "Boundary layer separation | Aerospace Engineering Blog," Aerospace Engineering Blog, 2016. [Online]. Available: http://aerospaceengineeringblog.com/boundary-layer-separation-and-pressure-drag/. [Accessed: 10-Jul-2017].

[37] L. Espeyrac And S. Pascaud, "Strouhal Instability - Von Karman Vortex Street," MFV Fluid Mechanics. [Online]. Available: http://hmf.enseeiht.fr/travaux/CD0102/travaux/optmfn/gpfmho/0102/grp1/presenta.htm. [Accessed: 06-Jul-2017].

[38] F. M. White, Viscous Fluid Flow. McGraw-Hill Higher Education, 2006.

[39] H. K. Versteeg and W. Malalasekera, An Introduction to Computational Fluid Dynamics The Finite Volume Method, vol. 572. Pearson Education Ltd, 1995.

[40] C. H. N. Yuen and R. F. Martinez-Botas, "Film cooling characteristics of rows of round holes at various streamwise angles in a crossflow: Part II. Heat transfer coefficients," Int. J. Heat Mass Transf., vol. 48, no. 23-24, pp. 5017-5035, Nov. 2005.

[41] J. Ahn, I. Sung Jung, and J. S. Lee, "Film cooling from two rows of holes with opposite orientation angles: Injectant behavior and adiabatic film cooling effectiveness," Int. J. Heat Fluid Flow, vol. 24, no. 1, pp. 91-99, Feb. 2003.

[42] C. M. Meador, "Massively-Parallel Direct Numerical Simulation of Gas Turbine Endwall Film-Cooling Conjugate Heat Transfer," Feb. 2010.

[43] E. Lutum, J. Von Wolfersdorf, K. Semmler, J. Dittmar, and B. Weigand, "Experimental investigation of film cooling on a convex surface subjected to favourable pressure gradient flow," Int. J. Heat Mass Transf., vol. 44, no. 5, pp. 939951, Mar. 2001. 
[44] M. Gritsch, A. Schulz, and S. Wittig, "Film-cooling holes with expanded exits: Nearhole heat transfer coefficients," Int. J. Heat Fluid Flow, vol. 21, no. 2, pp. 146-155, Apr. 2000.

[45] D. Whitlow, "Boundary Layer Suction on Low-Speed, Flat Plate Wind Tunnel Wind Tunnel Apparatus Boundary Layer Suction Research and Investigation," Morgantown, WV.

[46] Dantec Dyanamics, "Constant Temperature Anemometry," DantecDyanamics.com. [Online]. Available: https://www.dantecdynamics.com/docs/support-anddownload/research-and-education/cta.pdf. [Accessed: 11-Jul-2017].

[47] Dantec Dynamics, "Laser Doppler Anemometry," Dantec Dynamics.com. [Online]. Available: https://www.dantecdynamics.com/docs/support-and-download/researchand-education/lda.pdf. [Accessed: 11-Jul-2017].

[48] Dantec Dynamics, "Particle Image Velocimetry," Dantec Dynamics.com. [Online]. Available: https://www.dantecdynamics.com/docs/support-and-download/researchand-education/piv.pdf. [Accessed: 11-Jul-2017].

[49] J. Sauer, "The SUDI Turbulence Generator - A Method to Generate High Freestream Turbulence Levels and a Range of Length Scales," University of Wisconsin/University of Karlsruhe, Germany, 1996.

[50] J. B. Barlow, Low-speed wind tunnel testing. New York: Wiley, 1999.

[51] W. D. Baines and E. G. Peterson, "An Investigation of Flow Through Screens," Am. Soc. Mech. Eng., pp. 467-480, 1951.

[52] S. Andrew Hayes, A. C. Nix, C. M. John Kuhlman, and W. W. Huebsch, "An Experimental Investigation On the Effects of Freestream Turbulence Intensity On Film Cooling Effectiveness and Heat Transfer Coefficient for an Anti-Vortex Hole."

[53] F. E. Jørgensen, "How to measure turbulence with hot-wire anemometersa practical guide," Dantec Dyn., p. 3244, 2002.

[54] A. C. Nix, "Effects of High Intensity, Large-Scale Freestream Combustor Turbulence On Heat Transfer in Transonic Turbine Blades," Virginia Polytechnic Institute and State University, 2003. 